Vinícius Santos Andrade

\title{
Análise Dinâmica de uma Viga Engastada Excitada por uma Fonte Não Ideal.
}

\author{
Tese apresentada à Escola de Engenharia \\ de São Carlos, da Universidade de São \\ Paulo, como parte dos requisitos para \\ obtenção do título de Doutor em \\ Engenharia Mecânica.
}

Orientador:

Prof. Tit. Paulo Sérgio Varoto

São Carlos, SP 
Ao meu super pai José Pedro e grandes irmãos Chris e Léo. 


\section{Agradecimentos}

Agradeço ao Prof. Paulo Sérgio Varoto pela oportunidade e apoio dispensado durante toda a elaboração deste projeto.

Ao amigo Prof. José Manoel Balthazar pelo incentivo e por toda atenção dispensada para que este trabalho se concretizasse.

Ao Prof. Marcelo Trindade pelo apoio nos momentos mais importantes deste trabalho.

Ao amigo Lucas pela amizade e atenção dedicada neste trabalho.

Ao Prof. Bento pelo apoio e pelas excelentes discussões do trabalho.

As secretárias do departamento de Dinâmica Cristina e Ana Paula pela competência e paciência com todos os alunos.

Aos técnicos Xina, Serginho, Diego, Leandro e Flaudemir pela ajuda em todos os momentos deste trabalho.

Ao Leopoldo, pela paciência e grande ajuda na parte experimental.

A Andréia, por toda colaboração nas análises experimentais.

A todos os amigos conquistados no Laboratório de Dinâmica, Thiago, Marcelo, Carlão, Demian e Ana Lúcia.

A Vera Lúcia, Mari e Manu pelo companheirismo ao longo de minha vida.

Ao Pedro Henrique por me lembrar o quanto é bom ser criança e ao Lucas, que nem nasceu direito, mas já é tão importante na minha vida.

Agradeço a CAPES, pelo apoio financeiro através da bolsa de doutorado, sem a qual nenhum dos tópicos apresentados neste trabalho poderia ser desenvolvido.

Encerro fazendo um agradecimento especial e merecido a Tania, pelo apoio dedicado, pelas risadas e por sua enorme paciência, agradeço por me mostrar a leveza da vida e por estar sempre ao meu lado como minha mulher. 


\section{SUMÁRIO}

Resumo

Abstract

vii

Lista de Tabelas

viii

Lista de Figuras

ix

Lista de Símbolos

CAPÍTULO 1 - Introdução

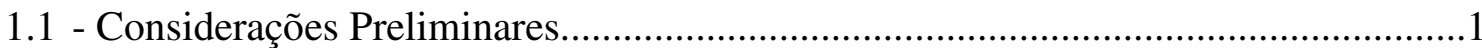

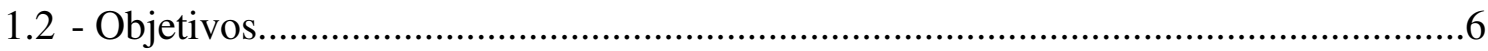

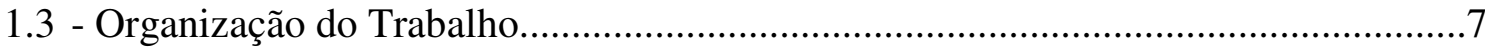

CAPÍTULO 2 - Revisão Bibliográfica 9

CAPÍTULO 3 - Modelagem Matemática do Sistema 20

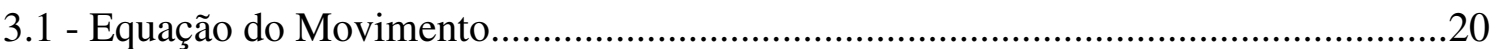

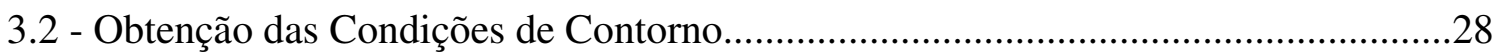

3.3 - Análise Numérica das Equações do Movimento....................................................29 
3.4.1 - Comparação com o Modelo de Duffing.......................................................35

3.4.2 - Sistema na Forma Espaço de Estado.......................................................36

CAPÍTULO 4 - Resultados de Simulações Numéricas $\quad 39$

4.1 - Análise Dinâmica do Sistema - Caso Ideal...........................................................39

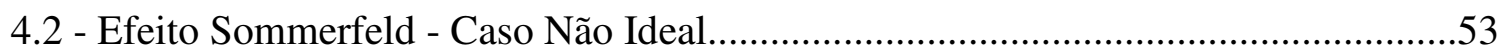

CAPÍTULO 5 - Ensaio Experimental $\quad 54$

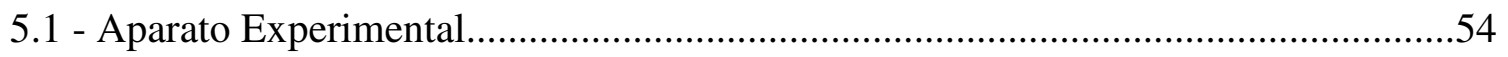

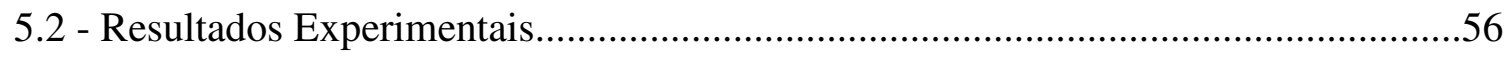

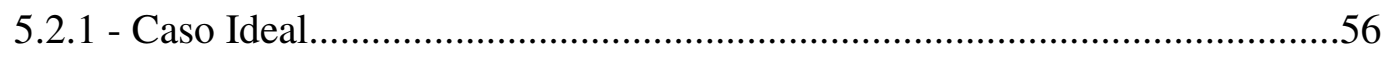

5.2.2 - Caso Não Ideal - Salto Experimental....................................................63

CAPÍTULO 6 - Conclusões e Sugestões para Trabalhos Futuros $\quad 66$

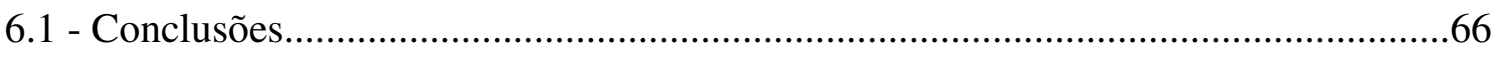

6.2 - Sugestões para Trabalhos Futuros.................................................................67

CAPÍTULO 7 - Referências Bibliográficas

Apêndice A - Método de Múltiplas Escalas $\quad 79$ 
Apêndice B - Desenvolvimento dos Cálculos Utilizando o Método dos Modos Assumidos nas Condições de Contorno

B.1 - Equações do Primeiro Modo de Vibrar.

Apêndice C - Desenvolvimento dos Cálculos: Equação do Momento Fletor $\tilde{M}$

Apêndice D - Parâmetros Físicos do Motor

Apêndice E - Reconstrução do Espaço de Fase

Apêndice F - Programas Utilizados nas Simulações Numéricas

C.1 - Programa Utilizado para o Cálculo do Expoente de Lyapunov. .97

C.2 - Programa Utilizado para o Cálculo do Histórico no Tempo e Plano de Fase.. 109

C.3 - Programa Utilizado para a Obtenção do Gráfico do Salto. 111 


\section{Resumo}

Andrade, V.S. (2009). Análise Dinâmica de uma Viga Engastada Excitada por uma Fonte Não Ideal. Tese (Doutorado) - Escola de Engenharia de São Carlos, Universidade de São Paulo.

Estudos sobre o comportamento dinâmico de estruturas não lineares são até os dias de hoje motivo de extensas pesquisas em todo o mundo. Desde o início do desenvolvimento da teoria das oscilações não lineares buscou-se compreender os mecanismos básicos, como perturbações que provocassem respostas complexas nas estruturas flexíveis. Este trabalho apresenta um estudo teórico e experimental do comportamento dinâmico de uma semi-asa de um avião acoplada a uma turbina com a hélice desbalanceada, esse sistema é representado através de uma viga engastada excitada por uma fonte não ideal localizada na extremidade oposta ao engaste. Entende-se como sistema não ideal aquele que considera que a excitação é influenciada pela própria resposta do sistema. Para sistemas dinâmicos não ideais, deve-se adicionar uma equação que descreva como a fonte não ideal interage com o sistema. Considera-se na equação do sistema apenas o primeiro modo de vibrar. Os resultados de simulação numérica apresentados são obtidos utilizando o software Matlab ${ }^{\circledR} 8.0$ e o parâmetro de controle a ser analisado é o torque do motor. Os resultados que mostram o comportamento dinâmico do sistema são o histórico no tempo, plano de fase, FFT e para identificar o comportamento caótico calculam-se os expoentes de Lyapunov. O gráfico que mostra a presença do efeito Sommerfeld (salto) no sistema também é apresentado. Na parte experimental, apresenta-se todo o procedimento experimental, assim como os resultados: Histórico no tempo, plano de fase reconstruído, FFT, expoentes de Lyapunov e as análises que ilustram a presença do efeito Sommerfeld no experimento.

Palavras Chave: Sistema não ideal, sistema não linear, efeito Sommerfeld, expoentes de Lyapunov. 


\begin{abstract}
Andrade, V.S. (2009). Dynamic Analysis of a Cantilever Beam Excited by a Non Ideal Source. Thesis (Doctorate) - São Carlos Engineering School, University of São Paulo.
\end{abstract}

Studies about the dynamic behaviour of nonlinear structures have been to this date subject of extensive research all around the world. Since the beginning of the development of the nonlinear oscillation theory one has tried to understand the basic mechanisms, like disruptions that would cause complex answers on flexible structures. This paper presents a theoretical and practical study of the dynamic behaviour of a semi-wing of an airplane installed on a turbine with unbalanced propellers; this system is represented through a cantilever beam excited by a non-ideal source located at the end opposite to the coupling. As a non-ideal system we mean the one that considers that the excitement is influenced by the system's response itself. For non-ideal dynamic systems, one must add an equation that describes how the non-ideal source interacts with the system. Only the first vibrating mode is considered in the system's equation. The numeric simulation results shown are obtained by using the Matlab ${ }^{\circledR} 8.0$ software and the control parameter to be analyzed is the motor torque. The results that show the dynamic behaviour of the system are time history, phase plan, FFT and to identify the chaotic behaviour the Lyapunov's indexes are calculated. The graphic that shows the presence of the Sommerfeld effect (jump) in the system is also presented. In the experimental part, all the practical procedure is presented, as well as experimental results, like, for example: Time history, phase plan reconstruction, FFT, Lyapunov exponents and the analyses that illustrate the presence of the Sommerfeld effect on the experiment.

Key words: Non ideal systems, nonlinear systems, Sommerfeld effect, Lyapunov exponents. 


\section{Lista de Tabelas}

Tabela 4.1 - Parâmetros do sistema.

Tabela 4.2 - Expoentes de Lyapunov para o torque do motor $T=0.5$.

Tabela 4.3 - Expoentes de Lyapunov para o torque do motor $T=1.5$.

Tabela 4.4 - Expoentes de Lyapunov para o torque do motor $T=2.5$.

Tabela 4.5 - Expoentes de Lyapunov para o torque do motor $T=4.5$.

Tabela 4.6: Expoentes de Lyapunov para o torque do motor $T=6.5$.

Tabela 4.7 - Sinais dos expoentes de Lyapunov para cada valor do torque do motor.

Tabela 5.1 - Parâmetros físicos do experimento.

Tabela 5.2 - Maior valor do expoente de Lyapunov para a tensão do motor $T=0.5 \mathrm{~V}$.

Tabela 5.3 - Maior valor do expoente de Lyapunov para a tensão do motor $T=1.5 \mathrm{~V}$.

Tabela 5.4 - Maior valor do expoente de Lyapunov para a tensão do motor $T=4.5 \mathrm{~V}$.

Tabela 5.5 - Maior valor do expoente de Lyapunov para a tensão do motor $T=6.5 \mathrm{~V}$. 


\section{Lista de Figuras}

Figura 1.1 - Sinais dos expoentes de Lyapunov para os atratores possíveis em $\mathfrak{R}^{3}$.

Figura 3.1 - Viga engastada com uma massa localizada na extremidade oposta ao engaste.

Figura 3.2 - Elemento infinitesimal da viga que mostra a projeção das deformações nos eixos.

Figura 3.3 - Momento Fletor $\tilde{M}$ do elemento infinitesimal da viga.

Figura 3.4 - Massa concentrada $m_{R B}$ acoplada à massa rotativa desbalanceada $m_{e}$.

Figura $4.1-T=0.5 \mathrm{~V}$. (a) Histórico no tempo. (b) Plano de fase. (c) FFT.

Figura 4.2 - Evolução dos expoentes de Lyapunov.

Figura $4.3-T=1.5 V$. (a) Histórico no tempo. (b) Plano de fase. (c) FFT.

Figura 4.4 - Evolução dos expoentes de Lyapunov.

Figura $4.5-T=2.5 \mathrm{~V}$. (a) Histórico no tempo. (b) Plano de fase. (c) FFT.

Figura 4.6 - Evolução dos expoentes de Lyapunov.

Figura $4.7-T=4.5 V$. (a) Histórico no tempo. (b) Plano de fase. (c) FFT.

Figura 4.8 - Evolução dos expoentes de Lyapunov.

Figura $4.9-T=6.5 \mathrm{~V}$. (a) Histórico no tempo. (b) Plano de fase. (c) FFT. 
Figura 4.10 - Evolução dos expoentes de Lyapunov.

Figura 4.11 - Efeito Sommerfeld.

Figura 5.1 - Esquema da montagem experimental.

Figura 5.2 - Fotos do experimento.

Figura $5.3-T=0.5 \mathrm{~V}$. (a) Histórico no tempo. (b) Plano de fase reconstruído, sendo $t$ a série temporal e $\tau$ a defasagem. (c) FFT.

Figura $5.4-T=1.5 \mathrm{~V}$. (a) Histórico no tempo. (b) Plano de fase reconstruído, sendo $t$ a série temporal e $\tau$ a defasagem. (c) FFT.

Figura $5.5-T=4.5 \mathrm{~V}$. (a) Histórico no tempo. (b) Plano de fase reconstruído, sendo $t$ a série temporal e $\tau$ a defasagem. (c) FFT.

Figura $5.6-T=6.5 \mathrm{~V}$. (a) Histórico no tempo. (b) Plano de fase reconstruído, sendo $t$ a série temporal e $\tau$ a defasagem. (c) FFT.

Figura 5.7 - Efeito Sommerfeld para o aumento e redução da voltagem do motor desbalanceado.

Figura 5.8 - (a) Variação da amplitude em função do tempo para o aumento da voltagem (Ida). (b) Amplitude versus Tempo para a redução da voltagem (Volta).

Figura A1 - Os três primeiros modos de vibrar da viga sem amortecimento e livre de esforços externos.

Figura C1 - Representação esquemática do elemento infinitesimal da viga para a obtenção da equação do momento. 
Figura E1 - Atrator caótico do modelo de Rössler.

Figura E2 - Histórico no tempo do atrator de Rössler.

Figura E3 - Reconstrução do atrator de Rössler por meio da defasagem da série temporal $x(t)$. 


\section{Lista de Símbolos}

$L=$ Comprimento da viga;

$m_{R B}=$ Massa rígida;

$\tilde{N}=$ Força axial;

$\tilde{x}=$ Eixo cartesiano horizontal;

$\tilde{y}=$ Eixo cartesiano vertical;

$\theta=$ Ângulo entre o eixo $\tilde{x}$ e linha neutra;

$\tilde{V}=$ Força de cisalhamento;

$\rho=$ Densidade da viga;

$\tilde{u}=$ Deslocamento axial;

$A=$ Área da seção transversal da viga;

$\widetilde{w}=$ Deslocamento transversal;

$d \widetilde{x}=$ Comprimento não deformado do elemento infinitesimal da viga;

$d \tilde{L}=$ Comprimento deformado do elemento infinitesimal da viga;

$\tilde{M}=$ Momento fletor;

$E=$ Módulo de elasticidade da viga;

$I$ = Momento de inércia da área da seção transversal da viga;

$R=$ Raio de curvatura da viga;

$m_{e}=$ Massa do desbalanceamento do motor;

$\vec{a}_{G}=$ Aceleração do centro de massa de $m_{R B} ;$

$\vec{a}_{e}=$ Aceleração do centro de massa de $m_{e} ;$

$M=$ Soma das massas $m_{R B}+m_{e}$ 
$\phi=$ Ângulo que caracteriza a posição da massa desbalanceada;

$I_{e}=$ Momento de inércia do motor;

$I_{R B}=$ Momento de inércia da massa rígida;

$T=$ Torque do motor;

$T_{E}=$ Tensão do motor;

$D=$ Atrito aerodinâmico;

$\vec{b}_{1}$ e $\vec{b}_{2}=$ Versores que definem a base que gira junto com o motor;

$q(t)=$ Coordenadas generalizadas dependentes do tempo;

$\Psi_{i}(x)=$ Funções de forma;

$e=$ Raio do disco acoplado ao motor;

$\Lambda=$ Expoente de Lyapunov;

$t=$ Série temporal obtida experimentalmente;

$\tau=$ Defasagem utilizada na reconstrução do espaço de fase. 


\section{Capítulo 1}

\section{Introdução}

\section{1 - Considerações Preliminares}

O desenvolvimento de máquinas e equipamentos que sejam mais eficientes, menos poluentes, mais seguros e, principalmente, mais baratos, sempre foi e sempre será o maior objetivo das indústrias e do mercado atual. Porém, para que todo desenvolvimento tecnológico seja alcançado, deve-se estudar, pesquisar e desenvolver soluções inovadoras a fim de melhorar a qualidade e a fabricação dos produtos.

Em projetos voltados à área de engenharia, podem ser citadas algumas aplicações, como por exemplo, projeto de aviões, turbinas, pontes, automóveis, etc. No entanto, para que essas aplicações sejam eficientes durante seu funcionamento, deve-se estudar e analisar um fenômeno comum a todas elas: a vibração estrutural.

A análise de qualquer problema que tenha como foco principal de estudo a vibração, pode ser abordado tanto na parte teórica, quanto na parte experimental. Porém, em muitos casos, somente a análise teórica pode ser estudada, devido a fatores climáticos, como o frio e o calor, que impossibilitam a realização experimental. Por outro lado, existem casos em que somente o estudo experimental pode ser feito devido à complexidade do problema, fazendo com que o modelo matemático não represente de forma real o experimento. Sendo assim, o comportamento dinâmico real de uma estrutura é acompanhado de análise teórica e experimental.

Nas análises teóricas e experimentais é comum observar-se a existência de correlação entre os resultados. Essas comparações entre os resultados podem tanto validar um modelo matemático, quanto indicar problemas na aquisição dos dados experimentais, ou até mesmo problemas nos equipamentos utilizados. 
Em geral, a principal etapa para estudo de um projeto de uma determinada estrutura sujeita às vibrações mecânicas é a determinação de suas frequiências naturais, que deve ser considerada para verificar a possibilidade da ocorrência do fenômeno da ressonância, a qual leva a grandes deformações e falhas mecânicas. Por exemplo, a investigação das vibrações que podem ocorrer em uma viga, excitada por motores de corrente contínua desbalanceados e com potência limitada tem uma considerável importância na engenharia mecânica atual. Estes problemas são denominados sistemas dinâmicos não ideais.

Nos casos em que a dinâmica da estrutura influencia a fonte de excitação e vice e versa são classificados como sistemas não ideais. Para os sistemas não ideais, as equações pertinentes ao sistema de excitação devem ser integradas juntamente com as equações do movimento da estrutura a fim de que o comportamento do sistema possa ser analisado (Balthazar 2003). Desta forma, torna-se necessário estabelecer uma formulação mais realista, que leve em conta a interação entre as variáveis de controle e as de estado da excitação com as de estado do sistema físico estrutural.

Os sistemas dinâmicos classificados como ideais são aqueles cuja excitação é conhecida e acima de tudo independente da dinâmica do sistema. Nestes casos a fonte de excitação influencia a dinâmica da estrutura sobre qual está atuando, mas a dinâmica dessa estrutura não influencia a dinâmica da excitação. Nos sistemas ideais a excitação é, em geral, uma função do tempo.

Uma máquina não ideal é uma conceituação que depende fundamentalmente da estrutura que a suporta e a sua relação "torque versus velocidade angular" é afetada, não mais correspondendo àquela deduzida estaticamente.

Um sistema não ideal típico, como um motor elétrico desbalanceado montado sobre uma base flexível, apresenta como resultado da interação da estrutura com a fonte de excitação, uma série de efeitos característicos, tais como (Balthazar, 2003) e (Fenili,2005):

- Variações bruscas (“jumps”, ou saltos) da amplitude de deslocamento da estrutura e da freqüência de excitação; 
No caso particular, em que se consideram as condições estacionárias de movimento:

- Descontinuidade da curva "resposta versus freqüência".

- Influência no perfil da curva "resposta versus freqüência", da forma pela qual se está alterando a velocidade do motor elétrico.

- Dependência destes efeitos das características eletromecânicas do motor elétrico.

Portanto, percebe-se que os sistemas dinâmicos, modelados matematicamente, como sendo não ideais, possuem pelo menos um grau de liberdade superior aos sistemas ideais correspondentes. Cabe ressaltar que, dependendo do número de motores presente no sistema, esse número de graus de liberdade pode aumentar.

Um exemplo clássico de sistema não ideal, que será estudado com detalhes nos tópicos que seguem, é o de uma estrutura flexível, ou seja, uma viga engastada onde um motor elétrico de corrente contínua e desbalanceado é colocado na extremidade livre da estrutura, como sendo uma fonte de potência limitada.

A viga é um dos elementos fundamentais de uma estrutura de engenharia, podendo-se modelar matematicamente diversos sistemas, como por exemplo uma semiasa de avião. Segundo Timoshenko (1953), as teorias para vigas começaram a ser estudadas a partir do século XVII. Em meados do século XVIII, Bernoulli e, principalmente, Eüler apresentaram trabalhos que podem ser considerados como o limiar da teoria geometricamente não linear para vigas com o material elástico linear. É evidente que tais teorias não continham o rigor nas conceituações como se verifica atualmente. Basicamente, pode-se definir a teoria não linear (seja de viga ou não) como sendo aquela que trata de não-linearidade geométrica, exata ou aproximada, com pequenas ou grandes deformações e nos regimes elástico (linear ou não), plástico e viscoelástico.

Entre os mais conhecidos modelos de viga estão os de Euler-Bernoulli, Vlasov e Timoshenko. No modelo de Eüler-Bernoulli, o cisalhamento e a inércia de rotação são desprezados, e supõe-se que as seções transversais planas permaneçam sempre planas e perpendiculares ao eixo longitudinal da viga, após sua deformação. No modelo de Vlasov, o cisalhamento continua não sendo considerado, porém é levada em conta a 
inércia de rotação. Por fim, no modelo de Timoshenko, supõe-se também que as seções transversais planas permaneçam planas, mas não necessariamente perpendiculares ao eixo longitudinal da viga, pois, devido ao cisalhamento, há um giro da seção em relação a essa perpendicular. É oportuno citar que, quando as dimensões da seção transversal são pequenas, em comparação ao seu comprimento, o modelo mais apropriado é o de EülerBernoulli.

Já nos casos em que as dimensões da viga não são pequenas, em comparação ao comprimento da viga (vigas curtas), situação em que o cisalhamento deve ser considerado, o modelo de Timoshenko é o mais indicado. Para altas freqüências, o modelo de Euler-Bernoulli não fornece bons resultados e, portanto, o modelo de Timoshenko deve ser usado, independente da geometria da viga.

Uma vez obtido o modelo matemático do sistema, pode-se, através de análises numéricas e experimentais, observar interessantes fenômenos físicos que podem ocorrer em estruturas tipo viga devido ao aparecimentos de não-linearidades, como por exemplo saltos, ressonâncias, interações modais, caos, etc. Na realidade, nenhum sistema físico é estritamente linear e, consequentente, modelos lineares de sistemas físicos apresentam muitas limitações. Em geral, modelos lineares só são aplicáveis em um domínio muito restrito, ou seja, onde a amplitude de vibração é muito pequena. Assim, para identificar com precisão e entender o comportamento dinâmico de um sistema estrutural, é essencial que as não-linearidades estejam presentes na estrutura modelada.

Teoricamente, não-linearidades aparecem nos sistemas sempre que há produtos de variáveis dependentes que derivam das equações do movimento, condições de contorno, saltos no sistema ou qualquer tipo de descontinuidade. Nayfeh e Mook (1979) e Moon (1987) explicaram em detalhes, junto com diversos exemplos, os vários tipos de nãolinearidades.

Em mecânica estrutural, as não linearidades podem ser classificadas como sendo devidas ao amortecimento, à geometria do sistema em estudo, inercial, condição de contorno, impacto, folgas mecânicas, entre outras. É interessante notar que a maioria de sistemas físicos pertence à classe de sistemas fracamente não lineares. Além disso, exibem fenômenos nos quais não existem no domínio linear. Sendo assim, para estruturas 
fracamente não lineares, utiliza-se como forma de análise a identificação das frequiências naturais lineares.

Em estruturas nas quais as não linearidades exercem uma influência significativa no sistema dinâmico, têm-se várias formas de análise numérica para avaliar a estabilidade do sistema tais como : plano de fase, mapa de Poincaré, diagrama de bifurcação, FFT (Fast Fourier Transform), expoentes de Lyapunov, etc. (Wolf 1985, Williams 1997, Parker, 1989).

A análise da estabilidade pode ser feita, através da perturbação do sistema com posterior observação de seu comportamento (Nayfeh 1985). Entende-se por perturbação qualquer alteração nos parâmetros do sistema, como por exemplo amortecimento, amplitude, freqüência, tensão, etc. Cabe ressaltar que, dependendo da magnitude da perturbação utilizada, o sistema dinâmico pode ou não perder sua estabilidade.

A motivação para se perturbar um sistema dinâmico está baseada na possibilidade de analisar a transição entre o comportamento do sistema, quando ele passa de estável a instável ou caótico. A principal análise para afirmar que um sistema apresenta um comportamento cáotico é o cálculo dos expoentes de Lyapunov, os quais medem a taxa de divergência de trajetórias e, portanto, permitem a análise da dependência sensitiva às condições iniciais. Além disso, a análise do sinal do expoente também permite identificar regiões caóticas e não-caóticas nos sistemas. Em outras palavras, um expoente de Lyapunov positivo indica que existe uma direção característica, na qual os atratores (regiões do espaço de fase que descrevem órbitas fechadas) tendem a se separar. Por outro lado, um expoente negativo indica que existe uma direção característica, na qual os atratores tendem a se aproximar.

Finalmente, o expoente de Lyapunov permite fazer uma análise do comportamento, seja ele caótico ou não-caótico, de trajetórias e seu sinal está intimamente ligado ao aparecimento de atratores nos sistemas, como o atrator ponto fixo, ciclo limite, toro e atrator estranho (Nayfeh e Balachandra 1995, Argyris 1994 e Ferrara 1995), como mostrado na figura, como segue. 


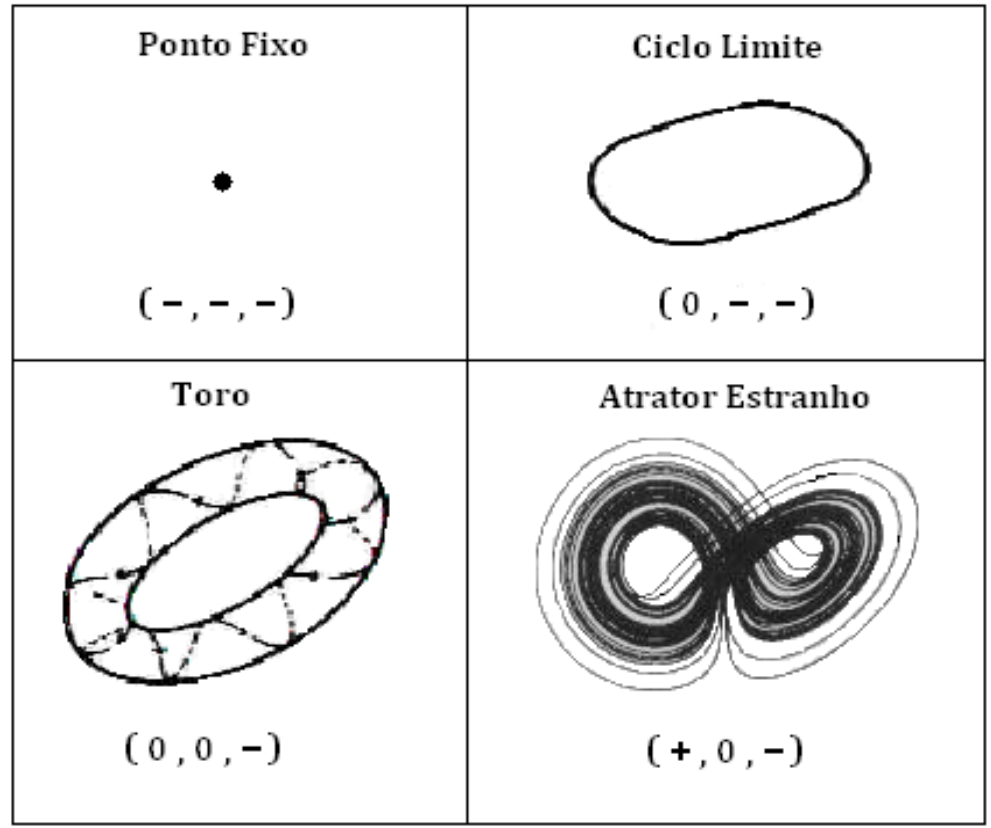

Figura 1.1 - Sinais dos expoentes de Lyapunov para os atratores possíveis em $\mathfrak{R}^{3}$. Como exemplo de atrator, utiliza-se o atrator de Lorenz (Ferrara, 1995).

\section{2 - Objetivos}

O presente trabalho tem como objetivo formular um modelo matemático detalhado de uma viga engastada com um motor desbalanceado e acoplado na extremidade oposta ao engaste. O modelo apresenta um conjunto mínimo de hipóteses simplificadoras para que, desta forma, obtenha-se um conjunto completo de equações, quando comparado com o sistema não ideal e não amortecido de Duffing. O sistema de equações apresentado é geral, ou seja, pode-se escolher neste sistema, a quantidade de modos de vibrar se pretender fazer as análises. Cabe ressaltar que, neste trabalho, será analisado apenas o primeiro modo de vibração.

Com o objetivo de analisar a perda de estabilidade estrutural do sistema, simulações numéricas serão realizadas. O software utilizado será o Matlab ${ }^{\circledR} 8$. O parâmetro de controle utilizado nas simulações será o torque do motor. 
A validação dos resultados teóricos será feita através dos resultados experimentais.

\section{3 - Organização do Trabalho}

O trabalho encontra-se dividido nos seguintes capítulos:

No Capítulo 2, é realizada uma revisão bibliográfica dos principais trabalhos utilizados como base para o desenvolvimento desta tese.

No Capítulo 3, apresenta-se a modelagem matemática de uma viga engastada com um motor desbalanceado, localizado na extremidade oposta ao engaste. A solução das equações do sistema será feita com a utilização do método de múltiplas escalas. A discretização das equações será realizada por meio do método dos modos assumidos para que, assim, possa-se realizar uma análise dinâmica por meio de simulações numéricas.

O Capítulo 4 apresenta uma análise dinâmica do sistema por meio dos resultados de simulação numérica, sendo que os resultados são divididos em caso ideal, onde os resultados apresentados são: Histórico no tempo, plano de fase, FFT e expoentes de Lyapunov e caso não ideal onde o resultado apresentado é o efeito Sommerfeld.

O procedimento experimental é apresentado no Capítulo 5, juntamente com os resultados experimentais, em que uma investigação dos efeitos não lineares presentes no sistema será realizada. Através da variação da tensão do motor pretende-se observar a presença do efeito Sommerfeld (salto) e, também, obter os gráficos que ilustram os efeitos da variação de amplitude em relação à tensão do motor, juntamente com histórico no tempo, plano de fase reconstruído (Simoni, 2008), FFT e expoentes de Lyapunov experimental (Simoni, 2008).

No Capítulo 6, são apresentadas as conclusões do trabalho e propostas futuras.

As referências bibliográficas são apresentadas no Capítulo 7.

No Apêndice A é apresentado o detalhamento dos cálculos, utilizando-se o método de múltiplas escalas para obter a solução do sistema livre.

No Apêndice B é apresentado o detalhamento dos cálculos, utilizando-se o método dos modos assumidos nas condições de contorno do sistema.

A demonstração da equação do momento fletor é apresentada no Apêndice C. 
Capítulo 1 - Introdução

As características físicas do motor utilizado nos ensaios experimentais são apresentadas no Apêndice D.

No Apêndice E é apresentada uma síntese da técnica de reconstrução do espaço de fase, utilizando-se como exemplo a reconstrução do atrator de Rössler.

O código dos programas utilizados nas simulações numéricas são apresentados no Apêndice F. 


\section{Capítulo 2}

\section{Revisão Bibliográfica}

Neste capítulo, é apresentada uma revisão bibliográfica dos principais trabalhos utilizados como base para a elaboração desta tese.

Não obstante o grande progresso da teoria de vibrações verifica-se que existem tipos especiais de vibrações que surgem da interação do sistema com a fonte de energia e não podem ser completamente explicadas pela teoria atual. Então, é conveniente usar a seguinte nomenclatura: Uma fonte de energia ideal atua na vibração do sistema sem sofrer qualquer influência do sistema; já uma fonte de energia não ideal atua na vibração do sistema e ao mesmo tempo sofre uma ação recíproca do sistema.

O primeiro relato que se tem a respeito da interação entre um sistema oscilante e a fonte de energia é apresentado por Sommerfeld em 1902. O fenômeno ficou conhecido com "salto" (jump), e atualmente denominado como efeito Sommerfeld.

Dois anos mais tarde (1904), Sommerfeld montou um motor elétrico em uma mesa flexível e, controlando a potência elétrica fornecida à máquina, monitorou a freqüência de excitação e a amplitude da resposta do sistema. Alterando a potência elétrica fornecida, Sommerfeld determinava a velocidade do motor e a freqüência das oscilações forçadas da mesa. A partir desses estudos, Sommerfeld observou que esta frequiência de oscilação permanecia praticamente constante na região de ressonância (região de valores próximos ao da freqüência natural da mesa), apesar de acréscimos significativos da amplitude de deslocamento do sistema. Em dado momento, assim que a amplitude máxima de oscilação era alcançada, um pequeno acréscimo na potência elétrica provocava uma brusca alteração na freqüência de excitação. Esta brusca alteração, verificada experimentalmente por Sommerfeld, ficaria então amplamente conhecida como fenômeno de salto (jump) no estudo de vibrações não lineares.

Stoker (1950) suspeitava que as vibrações lineares eram instáveis na faixa de freqüência entre os pontos onde ocorriam os saltos, porém não conseguiu demonstrar sua hipótese. Citou também que não havia na literatura qualquer prova rigorosa nesse sentido. 
Timoshenko e Young (1955) ao estudarem o problema de máquinas desbalanceadas sobre fundações elásticas com amortecimento, consideraram que a ação do torque da máquina era influenciada pela flexibilidade do sistema. Eles explicitaram uma forma de se obter o torque máximo devido a esta interação, não fazendo menção ao fenômeno de salto.

Em 1958, Kononenko, apud Kononenko (1969), pesquisou como uma fonte de excitação (motor) interage com alguns sistemas oscilatórios. Ele introduziu na equação de movimento as características estáticas do motor, na forma de uma função não linear da velocidade de rotação. A partir do estudo da estabilidade do movimento estacionário, foi possível estabelecer maior entendimento sobre a natureza do movimento instável, de forma a analisar possíveis saltos na vibração, e a observação da dependência da forma pela qual a velocidade da fonte de energia era alterada. Foram realizados muitos testes experimentais, os quais confirmaram os resultados obtidos pelo autor, além de confirmar a possibilidade de se aplicar as características estáticas referentes à fonte de excitação nas equações de movimento. Os resultados obtidos forneceram uma base para o método de análise da interação entre uma fonte de excitação e um sistema oscilatório.

Bodger, W. K. (1967) analisou a desaceleração de um sistema de um grau de liberdade que consiste num rotor desbalanceado acoplado sobre uma estrutura bi-apoiada. Em seus estudos, foi observado que o aumento do giro de rotação do rotor levava a saltos no sistema.

O fenômeno da interação de um sistema oscilatório com uma fonte de excitação, desconsiderando-se sua dependência com relação às propriedades do motor elétrico, encontra-se discutido em Kononenko (1969). Dentre outros pontos importantes, deu-se um destaque especial aos aspectos do efeito Sommerfeld, por meio da explicação de sua fenomenologia através dos ensaios experimentais. Neste trabalho, foram abordados os sistemas oscilatórios lineares com um grau de liberdade, os sistemas não lineares (divididos em sistemas com forças restauradoras não lineares, sistemas não lineares com oscilações excitadas parametricamente e sistemas auto-excitados) também com um grau de liberdade, bem como sistemas com muitos graus de liberdade, dentre outros.

Hoa (1979) analisou o comportamento de uma viga engastada em movimento rotativo com uma massa concentrada localizada na extremidade livre ao engaste. A pesquisa mostra que, quando o sistema gira com baixa velocidade, a inserção da massa concentrada na extremidade oposta ao engaste leva a uma diminuição nos valores das frequências naturais. Por outro lado, em altas velocidades de rotação, as frequências 
naturais aumentam de valor. $\mathrm{O}$ autor destaca que os resultados apresentados no trabalho têm aplicações em turbinas, helicópteros, entre outros, além de sugerir uma extensão do trabalho na análise das frequências de vibrações de lâminas flexíveis de ventiladores para refrigeração.

A modelagem matemática de uma viga engastada no formato de um "L", com duas massas concentradas em cada uma das extremidades da viga, foi realizada por Haddow (1984). Neste trabalho, a solução das equações do movimento, obtida via Lagrange, foram feitas utilizando-se o método de múltiplas escalas. $\mathrm{O}$ artigo analisa, teórica e experimentalmente, a interação entre os modos de vibração da viga, regiões de estabilidade e saltos no sistema.

Simo (1985) apresentou uma formulação teórica das equações do movimento de uma viga tridimensional. O trabalho considerou que os deslocamentos e as rotações da viga não podiam ser pequenos e um estudo da cinemática da deformação da viga foi apresentado. No entanto, o trabalho não obteve resultados de simulação numérica do sistema estudado.

Simo e Vu-Quoc (1986a) realizaram um estudo teórico da flexibilidade de uma viga plana sujeita a grandes oscilações. Os autores utilizaram conceitos não lineares para descrever os deslocamentos e deformações, devido ao movimento rotacional da viga. A formulação apresentada permitiu que as não linearidades geométricas pudessem ser tratadas separadamente nas equações, isto é, analisando-se dois campos vetoriais independentes, sendo o primeiro relacionado com o vetor posição e, o segundo, com a rotação da seção cruzada da viga. Os resultados de simulações numéricas são apresentados em Simo e Vu-Quoc (1986b). O trabalho analisa o acoplamento entre as vibrações com as grandes oscilações da viga. Os autores demonstram o comportamento dinâmico do sistema em diferentes tipos de exemplos. Os resultados de simulações numéricas apresentados mostram, também, a eficácia da formulação teórica, quando se analisa a dinâmica de multicorpos.

Crespo da Silva e Hodges (1986) formularam equações diferenciais para uma viga rotativa, considerando todas as contribuições possíveis devido às não linearidades cúbicas, e investigaram as influências desses termos no movimento de uma pá de helicóptero. Além disso, concluíram, também, que os termos cúbicos que contribuíam mais no sistema eram aqueles associados com a não linearidade geométrica presentes na equação do movimento. 
Simo e Vu-Quoc (1987) discutiram a relação entre os termos não lineares de deformação da viga, juntamente com o enrijecimento centrífugo devido ao seu movimento rotacional. O trabalho mostrou que os termos não lineares são responsáveis pela transferência de uma parte da energia de rotação para o movimento de flexão da viga.

Anos mais tarde, Crespo da Silva (1988) e Crespo da Silva et al. (1991) contribuíram com a formulação de equações que descreviam o movimento em duas direções (transversal e lateral) combinado com movimentos torsionais e axiais (flexuralflexural-torsional-extensional).

Pai e Nayfeh (1990) também desenvolveram um sistema de equações diferenciais do mesmo sistema apresentado por Crespo da Silva (1988). O sistema desenvolvido pelos autores descreve uma vibração de rastreamento e as equações do movimento possuem termos acoplados, juntamente com as não linearidades quadráticas e cúbicas devido à curvatura e inércia da viga.

Zavodney e Nayfeh (1989) fizeram um estudo teórico e experimental em finas vigas engastadas de aço e fibra de carbono. O sistema foi excitado axialmente pela base, e as equações do movimento foram obtidas, utilizando-se a teoria de Eüler-Bernoulli de tal forma que a massa tivesse uma posição arbitrária no sistema. Os resultados teóricos e experimentais como resposta-frequência e resposta-amplitude foram obtidos para três tipos de vigas e todas falharam por fadiga, devido ao nível de vibração ser muito intenso.

Utilizando a equação de Lagrange, Cartmel (1990) obteve as equações do movimento de uma viga na condição engastada-livre com uma massa concentrada na extremidade livre ao engaste, sendo excitada pela base e transversalmente à viga.

Krasnopolskaya e Shvets (1993) modelaram, matematicamente, um eletromotorpêndulo (mecanismo composto por um pêndulo simples cujo ponto de suporte do pêndulo é excitado por sistema biela manivela conectado a um motor de corrente contínua). Por meio de simulações numéricas, suas análises mostraram que a interação entre a fonte de excitação (motor) e a estrutura (pêndulo) poderia fazer o sistema perder estabilidade estrutural, levando a um comportamento caótico.

Da Nóbrega (1994) analisou a auto-sincronização de um sistema composto por dois motores elétricos de corrente contínua e desbalanceados. O trabalho apresentou algumas deduções matemáticas relacionadas com os parâmetros físicos do motor elétrico. 
O comportamento do sistema não ideal apresentou, a partir da variação dos parâmetros de controle, variações bruscas das velocidades angulares dos motores e da amplitude do deslocamento, sendo observado o efeito Sommerfeld.

Yamanaka et al. (1994) realizaram a modelagem matemática de uma viga engastada com um rotor na extremidade oposta ao engaste. A velocidade angular do rotor foi considerada constante e o rotor foi montado sobre a linha neutra da viga. Para a obtenção da equação do movimento, foi utilizado o princípio de Hamilton.

Berdichevskyo et al. (1995) realizaram uma análise da dinâmica de uma viga engastada-livre. Na extremidade livre da viga foi introduzida uma força harmônica de forma a excitar o sistema. $\mathrm{O}$ trabalho argumenta que a resposta dinâmica da viga com a introdução da excitação periódica pode ser descrita por meio da análise do potencial dinâmico do sistema. Os resultados de simulação numérica mostraram regiões caóticas em atratores e nos mapas de Poincaré. O trabalho ilustra, de forma lúdica, um mapa de Poincaré, que se assemelha ao rosto de um monstro, e o próprio autor denomina-o como uma "adorável criatura".

Anderson et al. (1996) realizaram uma investigação experimental e teórica, analisando o primeiro e o segundo-modo de vibrar de uma viga de aço engastada e excitada axialmente pela base. A inclusão de um amortecimento quadrático dentro do modelo analítico resultou em melhoria na qualidade dos resultados teóricos, quando comparado com os resultados experimentais. Os resultados experimentais mostraram que a curvatura da viga, freqüentemente ignorada nos trabalhos, exerce um papel significativo dentro da resposta do primeiro modo, e os termos devido às não linearidades inerciais possuem um papel fundamental na resposta do segundo modo e, portanto, não devem ser desprezados. O trabalho discute, também, a necessidade de considerar diferentes modelos de amortecimentos para se analisar diferentes modos de vibrar.

De Mattos e Balthazar (1997) realizaram um estudo experimental da vibração de um sistema dinâmico não ideal, o qual foi realizado utilizando-se um motor e uma massa. Neste sistema, a massa foi acoplada ao motor com o objetivo de excitar o sistema. $\mathrm{O}$ efeito Sommerfeld foi observado e concluiu-se que a extensão do salto tinha uma relação direta com o aumento do desbalanceamento da massa rotativa.

Esmailzadeh e Jalili (1998) analisaram a estabilidade de um sistema composto por uma viga engastada com uma massa concentrada na extremidade oposta ao engaste, sendo o sistema excitado pela base na direção horizontal (eixo x). O modelo de viga escolhido foi o modelo de Timoshenko, pois, nesse modelo, considera-se a inércia de 
rotação e as deformações de cisalhamento. Os resultados numéricos apresentados foram planos de fase e diagramas de estabilidade, de forma a concluir que, com o aumento da massa concentrada, a região de estabilidade do sistema é diminuída.

Belato apresentou em (1998) uma análise dinâmica de um sistema não ideal, constituído de um pêndulo simples excitado horizontalmente por um motor de corrente contínua com potência limitada, denominado de eletro-pêndulo. Os resultados de simulação numérica apresentados foram históricos no tempo, plano de fase, mapa de Poincaré, diagrama de bifurcação, expoente de Lyapunov e espectro de frequência. Os resultados mostraram regiões com comportamentos regulares e irregulares (caóticos) devido às alterações feitas no parâmetro de controle (tensão do motor).

Conrad e Morgül (1998) analisaram, matematicamente, a estabilidade de uma viga engastada com uma massa localizada na extremidade livre. Para estabilizar o sistema foi aplicado um controle de força na extremidade livre da viga para que a solução resultante fosse próxima de zero.

De Mattos e Balthazar (1999) mostraram os resultados obtidos, a partir da análise de um sistema dinâmico composto por um motor de corrente contínua com desbalanceamento de massa. Este sistema foi montado sobre um suporte, acoplado lateralmente por um amortecedor e uma mola, de tal forma que podia oscilar horizontalmente. Por se tratar de um sistema não ideal, os autores analisaram a interação entre a dinâmica da estrutura oscilante e a dinâmica do motor. Este trabalho baseou-se nas características do motor, pois nos trabalhos encontrados na literatura levam-se em conta somente as características das curvas do torque aplicado ao rotor, e o torque de resistência à rotação.

Fenili (2000) e Fenili et al. (2003), contribuíram de forma significativa para o estudo de modelos matemáticos denominados de estruturas flexíveis de rastreamento (trata-se de um movimento em torno de um eixo perpendicular ao plano no qual o movimento se realiza, com por exemplo àquele realizado por uma porta quando gira em torno de suas dobradiças) e análises numéricas e experimentais de ressonâncias primárias e secundárias. Nestes sistemas, um deles foi considerado com comportamento ideal e o outro modelo como sendo não-ideal. Dois diferentes modelos matemáticos foram propostos, curvatura linear e curvatura não linear para modelar a deflexão de uma estrutura flexível de rastreamento (tipo viga). Para auxiliar na modelagem dos sistemas, um protótipo experimental foi construído e um novo modelo matemático para o atrito interno do motor utilizado na parte experimental foi apresentado. 
O sistema não ideal analisado por Belato (2001) foi o pêndulo eletro-mecânico. As oscilações deste sistema foram causadas por meio da variação da tensão do motor, a qual foi considerada como parâmetro de controle do sistema. Com a variação do parâmetro de controle, o sistema foi perdendo estabilidade estrutural e desta forma apresentou diferentes tipos de comportamentos, tais como o efeito Sommerfeld, bifurcações e caos.

Palacios (2002) investigou o comportamento dinâmico não-linear em um pórtico plano, o qual fora excitado por uma e depois por duas fontes não ideais (motor elétrico de corrente contínua e desbalanceado). As equações do movimento foram obtidas por meio da formulação Lagrangeana, considerando as não linearidades do sistema até ordem cúbica. Os resultados de simulação numérica mostraram fenômenos não lineares como salto, transferência de energia entre modos, saturação modal, ressonância interna 1:2. Além disso, um controle por saturação modal é estudada via Simulink.

A diversidade dos trabalhos publicados nessa área é muito grande, desta forma, pode-se citar uma revisão completa de diferentes teorias sobre sistemas vibrantes não ideais, tal como a que foi apresentada por Balthazar et al. (2003). Este trabalho apontou que a dinâmica entre a fonte de energia e a resposta estrutural não devem ser ignoradas em problemas de engenharia, considerando que os sistemas tenham fonte com potência limitada.

Dantas e Balthazar (2003) estudaram um sistema não ideal, que consiste em um motor desbalanceado apoiado numa base suportado por uma mola, fazendo com que o conjunto oscile verticalmente. Através de estudos de teoremas matemáticos, o trabalho discute que a ocorrência da bifurcação de Hopf é uma propriedade comum em problemas não ideais relacionado com o efeito Sommerfeld.

Os trabalhos de Pontes (2000) e (2003) apresentaram os resultados do estudo da dinâmica e do controle de um sistema não ideal auto-excitado pelo atrito, ou seja, quando existe uma interação do sistema com sua fonte de energia. Foram analisados os comportamentos dos sistemas ideal e não ideal por meio de simulação numérica. Apresentou-se também os resultados de uma estratégia de controle chaveado para posicionamento e para seguimento de trajetórias periódicas, controle do movimento caótico e a análise de estabilidade pelo método de Lyapunov.

Palacios (2003) utilizou as equações simplificadas de Duffing para analisar a dinâmica não linear de um sistema constituído por uma viga engastada e dois motores com massas desbalanceadas na extremidade livre. Os dois motores mostraram que a auto- 
sincronização podia ocorrer quando as velocidades de rotação dos motores se tornavam temporariamente próximas uma da outra, dependendo do torque aplicado. Foi mostrado também que, caso a variação do torque seja constante, era possível controlar a autosincronização e sincronização no sistema.

Tsuchida (2003) estudou um problema não ideal com dois graus de liberdade operando próxima à ressonância. Numa primeira análise, considerou-se o torque gerado pelo motor como sendo linear e, na sequiência, considerou-se o torque como sendo não linear. A partir das análises de vibração, estudou-se a possibilidade da existência de movimentos regulares e irregulares no sistema.

Dois anos mais tarde, o mesmo autor - Tsuchida (2005) - analisou o problema anteriormente descrito, porém com outro tipo de ressonância interna, 1:2. À medida que a frequência do rotor era aumentada, foram observados movimentos caóticos e o efeito Sommerfeld contribuiu para que o sistema apresentasse esse tipo de movimento. Cabe destacar, ainda, que a inclusão de termos quadráticos e cúbicos no sistema fez com que a vibração se tornasse mais complexa.

Fenili e Balthazar (2005) modelaram matematicamente uma viga engastada, cujo engaste apresentava um movimento rotativo (estrutura flexível de rastreamento). Nesse modelo, foi considerada uma curvatura não linear da viga, incluindo termos cúbicos. Os resultados de simulação numérica do sistema considerado como sendo ideal e depois como não-ideal foram comparados, dando um destaque especial para a importância de se considerar a influência da interação da fonte de energia com o movimento do sistema em alguns casos críticos.

Gomes da Silva (2005) realizou uma análise teórica e experimental em estruturas tipo viga. Foi analisada a influência da viscosidade na vibração e da presença de excitações combinadas nas vibrações ressonantes paramétricas. Foram analisadas vibrações de duas estruturas presentes numa aeronave. Inicialmente, estudou-se um sistema que tivesse o comportamento de um estabilizador vertical (aerofólio de perfil simétrico, que tem como finalidade evitar que a aeronave derrape durante uma curva). A outra estrutura analisada foi uma simplificação do conjunto asa/pilone/turbina. Por possuir uma estrutura muito complexa, a modelagem foi feita utilizando-se o método de elementos finitos. Na parte experimental, foram feitas análises de identificação modal, ensaios não lineares e vibrações autoparamétricas. 
A análise do movimento caótico de uma viga engastada sujeita à ação de uma excitação harmônica axial e uma excitação transversal na extremidade livre da viga foi apresentada por Zhang (2005). O trabalho ainda apresenta a aplicação de um novo tipo de controle de caos, usando aproximações numéricas.

Um novo método para obter a solução de um sistema de $4^{\mathrm{a}}$ ordem, que consiste de uma viga engastada com uma massa concentrada na extremidade livre, foi apresentada por Adomian (2007). Na obtenção das frequências naturais do sistema foi feita uma comparação da precisão do método proposto pelo autor com o método dos elementos finitos, obtendo-se resultados satisfatórios na comparação.

Claeyssen (2006) analisou um modelo de viga Eüler-Bernoulli do tipo "clampedfree" com um rotor em uma das extremidades livres. A partir da análise do problema livre, os modos de vibração do sistema foram obtidos de forma precisa. Considerou-se o sistema como um modelo distribuído para deslocamentos nas direções transversais da viga. O método de Ritz se mostrou satisfatório para os cálculos das simulações numéricas.

Bolla (2007) analisou um modelo simplificado de um sistema não ideal e não linear, utilizando o método de múltiplas escalas. Considerou-se no modelo as não linearidades quadráticas do motor DC e as não linearidades cúbicas da mola, ambas com a mesma ordem de grandeza. Observou-se o efeito Sommerfeld nos resultados de simulação numérica.

El-Badawy (2007), analisou um sistema representado por uma viga engastada com um motor desbalanceado na extremidade livre. O sistema de equações proposto por Kononenko (1969) foi utilizado como base nas análises matemáticas da solução do sistema. Na parte experimental, a freqüência natural do sistema foi determinada, utilizando-se um martelo de impacto e a interação entre o motor e a viga foi determinada analisando-se a variação entre a tensão do motor e a amplitude. Conforme esperado, o efeito Sommerfeld foi obtido.

Piccirillo (2008) analisou a dinâmica não linear de uma viga engastada com uma massa concentrada na extremidade livre, sendo utilizada na viga uma liga de memória de forma (SMA - shape memory alloy) e um amortecedor. Neste sistema, um motor é acoplado à massa de forma a causar interação entre a estrutura e a fonte de energia. $\mathrm{O}$ elemento SMA é caracterizado, utilizando-se um modelo constitutivo fenomenológico unidimensional. Os resultados de simulação numérica mostraram além do efeito 
Sommerfeld, a presença de caos e hipercaos, os quais foram detectados por meio do cálculo dos expoentes de Lyapunov.

De Souza (2007) estudou a dinâmica caótica de uma estrutura não linear e não ideal composta por uma mola e um amortecedor engastados num suporte vertical. Foi acoplada a esse conjunto uma massa juntamente com o motor desbalanceado de forma a executarem um deslocamento axial. Os resultados de simulação numérica mostraram que esse tipo de sistema apresentou movimento caótico. Uma técnica de controle de caos foi aplicada ao sistema, mostrando que foi possível controlá-lo.

Zukovic (2007) modelou matematicamente um sistema não linear e não ideal. O modelo era constituído por motor desbalanceado acoplado a uma massa, ambos apoiados sob uma mola e um amortecedor apresentavam apenas um deslocamento vertical. Neste trabalho, a dinâmica caótica do sistema foi analisada por meio da variação do parâmetro de controle, a qual era dependente das características do motor. Fenômenos não lineares tais como efeitos Sommerfeld, diagrama de bifurcação e expoentes de Lyapunov foram apresentados nos resultados de simulação numérica. Para o sistema não entrar em regime caótico, um método de controle de caos é aplicado ao sistema.

Dois anos mais tarde, Zukovic (2009) acrescentou algumas mudanças em seu sistema, o qual foi anteriormente descrito. Uma folga foi acrescentada ao modelo, engastou-se a mola e o amortecedor num suporte vertical e a massa, juntamente com o motor, passou a executar um deslocamento axial. A dinâmica foi feita por meio de simulações numéricas, obtendo-se como resultado o efeito Sommerfeld, diagrama de bifurcação e expoentes de Lyapunov. Foi concluído que, devido à folga, a interrupção no movimento oscilatório do sistema não ocorria, mas este movimento foi dividido em intervalos com e sem força elástica, o que causava perturbação na periodicidade do movimento. Um controle de caos também foi apresentado no trabalho.

Palacios et. al. (2009) realizaram uma investigação analítica e numérica na interação dinâmica entre uma viga engastada com um motor acoplado na extremidade livre, o amortecimento e a rigidez foram analisados utilizando o modelo de DuffingRayleigh. O efeito Sommerfeld foi observado, porém o artigo mostra que, a partir das análises feitas nos parâmetros de rigidez e amortecimento, é possível determinar as condições em que o sistema não apresenta o efeito Sommerfeld.

Observa-se ao longo dessa revisão bibliográfica que há diversas linhas de pesquisas envolvendo um mesmo tipo de problema, um motor desbalanceado acoplado a 
uma viga engastada, assim há muito a ser pesquisado sobre o tema de forma a contribuir com a literatura corrente.

No capítulo que segue apresenta-se a modelagem matemática de uma viga engastada com um motor desbalanceado acoplado na extremidade oposta ao engaste. 


\section{Capítulo 3}

\section{Modelagem Matemática do Sistema}

Neste capítulo são derivadas as equações do movimento e as condições de contorno para as vibrações forçadas de uma viga engastada com um motor desbalanceado na extremidade livre. A modelagem é iniciada com a obtenção das equações do movimento da viga e na seqüência o motor desbalanceado é introduzido ao modelo de forma a obter um sistema de equações resultante da interação do movimento da viga com o movimento do motor desbalanceado. As condições de contorno e a solução do sistema são calculadas de forma a obter um sistema geral de equações, sendo que esse sistema permite escolher com quantos modos de vibrar se deseja fazer as análises (Craig, 1981, Nayfeh e Mook, 1979, Nayfeh, 1985, Timoshenko, 1955). Nesse trabalho será analisado o primeiro modo de vibrar.

\subsection{Equação do Movimento}

Nesta primeira seção é feita a derivação das equações do movimento de uma viga de comprimento $L$ apoiada em uma massa rígida $m_{R B}$ na extremidade livre, é mostrada na como segue na Figura 3.1.

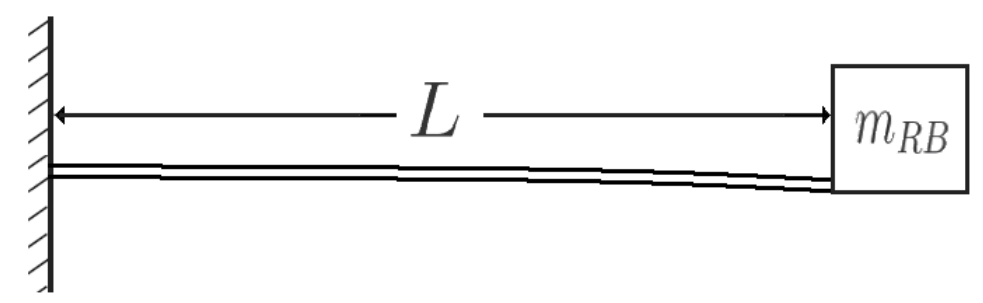

Figura 3.1 Viga engastada com uma massa localizada na extremidade oposta ao engaste. 
As hipóteses apresentadas nessa seção estão baseadas na teoria de EulerBernoulli para vigas, onde as seções planas permanecem planas e perpendiculares a linha neutra da viga (Timoshenko, 1955).

A Figura 3.2 mostra todas as componentes das forças $\tilde{x}$ e $\tilde{y}$ que agem no elemento infinitesimal da viga, como segue.

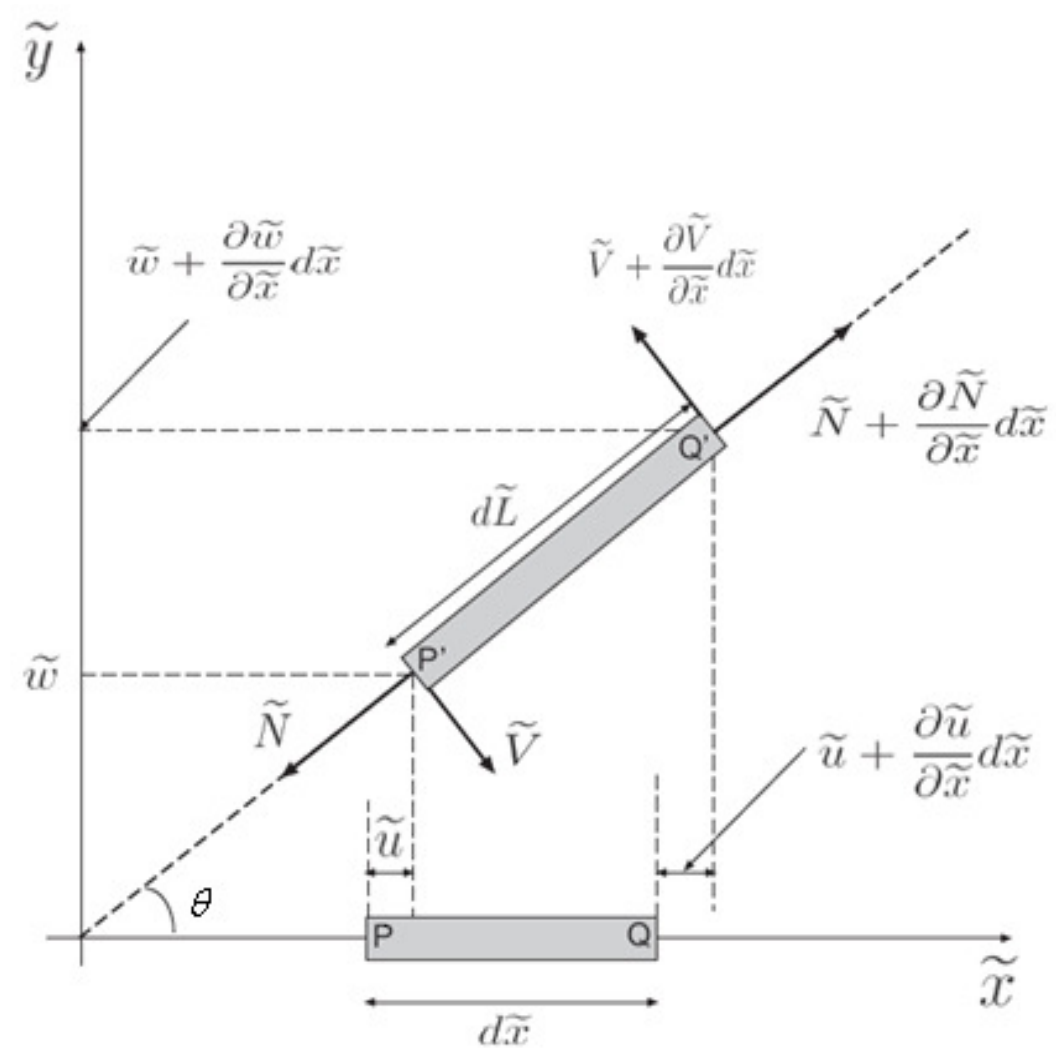

Figura 3.2 - Elemento infinitesimal da viga que mostra a projeção das deformações nos eixos.

Para o elemento de viga mostrada na Figura 3.2, observa-se que os pontos P e Q, que inicialmente estavam posicionados sobre o eixo-x, tornam-se P' e Q' quando a viga sofre um deslocamento transversal e o comprimento original $d \tilde{x}$ passa a ser $d \tilde{L}$.

Fazendo a somatória das componentes $\tilde{x}$ e $\tilde{y}$ da força, têm-se as equações abaixo, sendo que as variáveis são definidas em função de $\tilde{x}$ (viga não deformada) e $\tilde{t}$ (tempo).

$$
\begin{aligned}
& \frac{\partial}{\partial \tilde{x}}[\tilde{N} \cos \theta-\tilde{V} \sin \theta]=\rho A \frac{\partial^{2} \tilde{u}}{\partial \tilde{t}^{2}} \\
& \frac{\partial}{\partial \tilde{x}}[\tilde{N} \sin \theta+\tilde{V} \cos \theta]=\rho A \frac{\partial^{2} \tilde{w}}{\partial \tilde{t}^{2}}
\end{aligned}
$$


onde

- $\tilde{N}$ é a força axial;

- $\theta$ é o ângulo entre o eixo $\tilde{x}$ e linha neutra;

- $\tilde{V}$ é a força de cisalhamento;

- $\rho$ é a densidade da viga;

- $\tilde{u}$ é o deslocamento axial;

- A é a área da seção transversal da viga;

- $\tilde{w}$ é o deslocamento transversal.

A Figura 3.3 mostra os momentos do centro de massa do elemento infinitesimal da viga, como segue

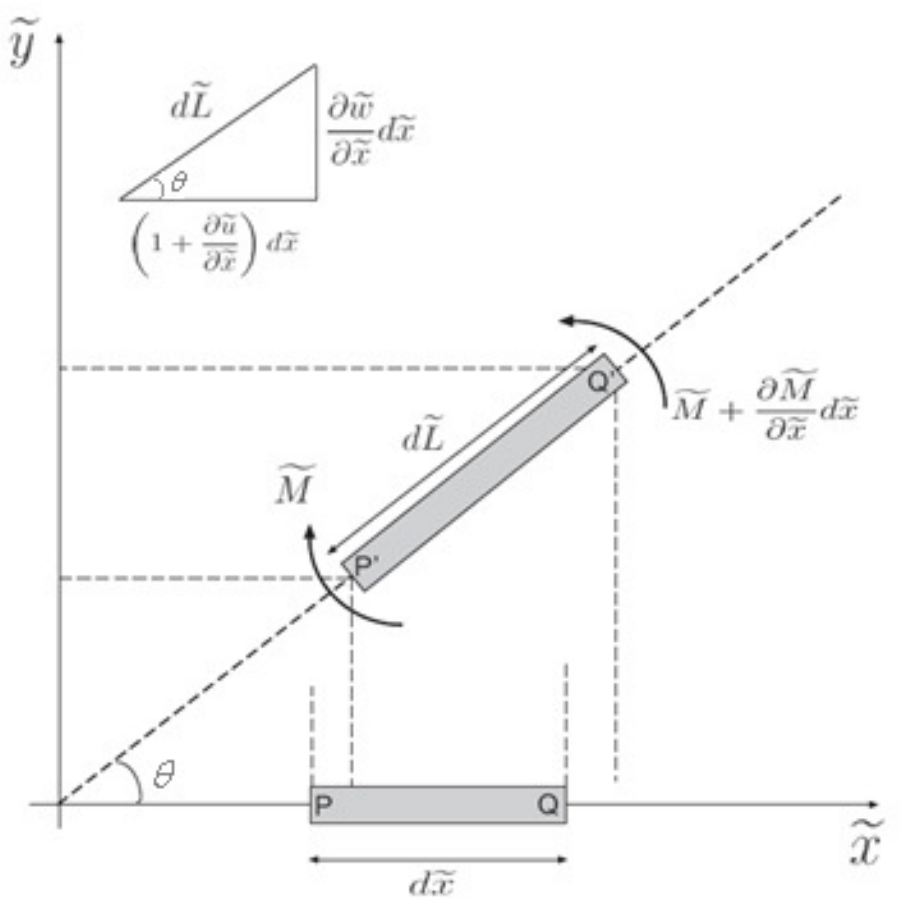

Figura 3.3 - Momento Fletor $\tilde{M}$ do elemento infinitesimal da viga.

Somando os momentos em torno do centro de massa do elemento infinitesimal da viga e assumindo que o momento angular seja desprezível quando comparada com a inércia linear (massa da viga), tem-se.

$$
\frac{\partial \tilde{M}}{\partial \tilde{x}}+\tilde{V} \frac{\partial \tilde{L}}{\partial \tilde{x}}=0
$$

onde $\tilde{M}$ é o momento fletor, $d \widetilde{x}$ é o comprimento não deformado do elemento e $d \tilde{L}$ é o deformado. 
De acordo com a Figura 3.3 obtém-se $d \tilde{L}$ como segue.

$$
d \tilde{L}=\left[\left(1+\frac{\partial \tilde{u}}{\partial \tilde{x}}\right)^{2}+\left(\frac{\partial \tilde{w}}{\partial \tilde{x}}\right)^{2}\right]^{1 / 2} d \tilde{x}
$$

O momento fletor é relacionado com a deflexão da viga como segue (Timoshenko, 1955), (Beer, 1995).

$$
\tilde{M}=E I \frac{\partial^{2} \tilde{w}}{\partial \tilde{x}^{2}}\left[1+\left(\frac{\partial \tilde{w}}{\partial \tilde{x}}\right)^{2}\right]^{-3 / 2}
$$

sendo $E$ o módulo de elasticidade e $I$ o momento de inércia da área da seção transversal da viga. A demonstração da Equação (3.1.5) é apresentada no Apêndice C.

Neste momento é conveniente introduzir variáveis adimensionais como abaixo (Fenili, 2000).

$$
x=\frac{\tilde{x}}{L} ; \quad \beta w=\frac{\tilde{w}}{L} ; \quad \beta^{2} u=\frac{\tilde{u}}{L} ; \quad t=\tilde{t} \sqrt{\frac{\beta E}{\rho L^{2}}} ; \quad \beta=\left(\frac{L}{R}\right)^{2} .
$$

Onde $R$ é o raio de curvatura da viga. Nota-se que $\beta$ é o quadrado do índice de esbeltez $(L / R)$ da viga (Timoshenko, 1961).

O parâmetro $\beta$ representa um pequeno parâmetro que será introduzido nas equações do movimento com o intuito de possibilitar a aplicação da teoria de perturbação, pois com as equações na forma perturbada, a ordem dos efeitos não lineares será aparente e o sistema de equações não lineares poderá ser resolvido por algum método conhecido de perturbação (Nayfeh e Mook, 1979). Como a solução das equações diferenciais exatas mostra-se muito complexa, utiliza-se nas equações do movimento a teoria de perturbação para obter equações aproximadas para o problema. Tais equações tornam possíveis as análises numéricas desejada. Observa-se na Equação (3.1.6) que $\beta$ está elevado a primeira potência na definição da variável $w$, enquanto que na definição de $u$, temos $\beta^{2}$. Estas definições são justificadas pelo fato de considerarmos o deslocamento axial da viga desprezível quando comparado a seu deslocamento transversal.

Substituindo a Equação (3.1.6) na Equação (3.1.4) e na Equação (3.1.5) e mantendo termos até terceira ordem em $\beta$ tem-se.

$$
\frac{d \tilde{L}}{d \tilde{x}}=1+\beta^{2}\left[\frac{\partial u}{\partial x}+\frac{1}{2}\left(\frac{\partial w}{\partial x}\right)^{2}\right]+O\left(\beta^{4}\right)
$$

e 


$$
\tilde{M}=\frac{E I}{L} \beta\left[\frac{\partial^{2} w}{\partial x^{2}}-\beta^{2} \frac{3}{2}\left(\frac{\partial w}{\partial x}\right)^{2}\right]+O\left(\beta^{4}\right)
$$

Substituindo Equação (3.1.7) e Equação (3.1.8) na Equação (3.1.3) tem-se a expressão para $\tilde{V}$ como segue.

$$
\tilde{V}=-\beta \frac{E I}{L^{2}}\left[\frac{\partial^{3} w}{\partial x^{3}}-\beta^{2} \frac{3}{2} \frac{\partial}{\partial x}\left(\frac{\partial w}{\partial x}\right)^{2}\right]
$$

Assume-se que a força axial $\tilde{N}$ é proporcional a tensão axial, como abaixo.

$$
\tilde{N}=E A \frac{d \tilde{L}-d \tilde{x}}{d x}=E A\left(\frac{d \tilde{L}}{d \tilde{x}}-1\right)
$$

Desta forma a Equação (3.1.10) pode ser escrita da seguinte forma.

$$
\tilde{N}=\beta^{2} E A\left(\frac{\partial u}{\partial x}-\frac{1}{2}\left(\frac{\partial w}{\partial x}\right)^{2}\right)
$$

De acordo com a Figura 3.3 e Equação (3.1.6) obtém-se as funções $\sin \theta$ e $\cos \theta \operatorname{como}$ segue.

$$
\sin \theta=\beta \frac{\partial w}{\partial x}-\frac{\beta^{3}}{2}\left(\frac{\partial w}{\partial x}\right)^{3}+O\left(\beta^{5}\right)
$$

$\mathrm{e}$

$$
\cos \theta=1-\frac{1}{2} \beta^{2}\left(\frac{\partial w}{\partial x}\right)^{2}+O\left(\beta^{4}\right)
$$

Para $\theta$ pequeno tem-se que $\sin \theta \cong \theta$, desta forma tem-se

$$
\theta=\beta \frac{\partial w}{\partial x}-\frac{\beta^{3}}{2}\left(\frac{\partial w}{\partial x}\right)^{3}+O\left(\beta^{5}\right)
$$

Da Equação (3.1.6), Equação (3.1.7) e Equação (3.1.10) obtém-se que

$$
\partial \tilde{N} / \partial x=O\left(\beta^{3}\right) \text {. }
$$

Para determinar a expressão da força axial $\tilde{N}$, considera-se a massa rígida $m_{R B}$ acoplada a uma pequena massa rotativa $m_{e}$ que está presa a viga em $x=L$ como mostra a Figura 3.4. 
Figura 3.4 - Massa concentrada $m_{R B}$ acoplada à massa rotativa desbalanceada $m_{e}$.

O somatório das forças resulta nas expressões como seguem

$$
\tilde{N} \cos \theta-\tilde{V} \sin \theta=\left[m_{R B} \vec{a}_{G}+m_{e} \vec{a}_{e}\right] \cdot \vec{i}
$$

e

$$
\tilde{N} \sin \theta+\tilde{V} \cos \theta=\left[m_{R B} \vec{a}_{G}+m_{e} \vec{a}_{e}\right] \cdot \vec{j}
$$

onde $\vec{a}_{G}$ e $\vec{a}_{e}$ são as acelerações do centro de massa de $m_{R B}$ e da massa rotativa e desbalanceada $m_{e}$, respectivamente.

Multiplicando a Equação (3.1.16) por $\cos \theta$ e a Equação (3.1.17) por $\sin \theta$ e somando os resultados, tem-se a equação para a força axial $\tilde{N}$.

$$
\begin{aligned}
& \left\{\begin{array}{l}
\tilde{N} \cos ^{2} \theta-\tilde{V} \sin \theta \cos \theta=\left(m_{R B} \vec{a}_{G}+m_{e} \vec{a}_{e}\right) \vec{i} \cos \theta \\
\tilde{N} \sin ^{2} \theta+\tilde{V} \cos \theta \sin \theta=\left(m_{R B} \vec{a}_{G}+m_{e} \vec{a}_{e}\right) \vec{j} \sin \theta
\end{array}+\right. \\
& \tilde{N}=\vec{i}\left(m_{R B} \vec{a}_{G}+m_{e} \vec{a}_{e}\right) \cos \theta+\vec{j}\left(m_{R B} \vec{a}_{G}+m_{e} \vec{a}_{e}\right) \sin \theta
\end{aligned}
$$

Assim, segue que $\vec{a}_{G}$ e $\vec{a}_{e}$ podem ser escritos em termos das coordenadas vetoriais como segue (Butkov, 1988, Arfken, 1995).

$$
\begin{aligned}
\vec{a}_{G}= & \vec{i} \frac{\partial^{2} \tilde{u}(\tilde{x}, \tilde{t})}{\partial \tilde{t}^{2}}+\vec{j} \frac{\partial^{2} \tilde{w}(\tilde{x}, \tilde{t})}{\partial \tilde{t}^{2}}+\frac{\partial^{2} \theta(\tilde{x}, \tilde{t})}{\partial \tilde{t}^{2}} \vec{k} \times\left[d_{1} \vec{b}_{1}+d_{2} \vec{b}_{2}\right] \\
& -\left(\frac{\partial \theta(\tilde{x}, \tilde{t})}{\partial \tilde{t}}\right)^{2}\left[d_{1} \vec{b}_{1}+d_{2} \vec{b}_{2}\right]
\end{aligned}
$$


Sendo que cada termo da Equação (3.1.19) é descrito como abaixo.

$\frac{\partial^{2} \tilde{u}(\tilde{x}, \tilde{t})}{\partial \tilde{t}^{2}} \vec{i}=$ aceleração na direção $i$.

$\frac{\partial^{2} \tilde{w}(\tilde{x}, \tilde{t})}{\partial \tilde{t}^{2}} \vec{j}=$ aceleração na direção $j$.

$\frac{\partial^{2} \theta(\tilde{x}, \tilde{t})}{\partial \tilde{t}^{2}} \vec{k} \times\left[d_{1} \vec{b}_{1}+d_{2} \vec{b}_{2}\right]=$ aceleração angular.

$\left(\frac{\partial \theta(\tilde{x}, \tilde{t})}{\partial \tilde{t}}\right)^{2}\left[d_{1} \vec{b}_{1}+d_{2} \vec{b}_{2}\right]=$ aceleração centrípeta.

$\mathrm{e}$

$$
\begin{aligned}
\vec{a}_{e}= & \vec{a}_{G}+\vec{k} \frac{\partial^{2} \theta(\tilde{x}, \tilde{t})}{\partial \tilde{t}^{2}} \times\left[P_{1} \vec{b}_{1}+P_{2} \vec{b}_{2}\right]-\left(\frac{\partial \theta(\tilde{x}, \tilde{t})}{\partial \tilde{t}}\right)^{2}\left[P_{1} \vec{b}_{1}+P_{2} \vec{b}_{2}\right] \\
& +\vec{k} \frac{d^{2} \phi(\tilde{t})}{d \tilde{t}^{2}} \times\left[\vec{b}_{1} e \cos \phi+\vec{b}_{2} e \sin \phi\right]-\left(\frac{d \phi}{d \tilde{t}}\right)^{2}\left[\vec{b}_{1} e \cos \phi+\vec{b}_{2} e \sin \phi\right]
\end{aligned}
$$

Cada termo da equação acima é descrito como segue.

$\frac{\partial^{2} \theta(\tilde{x}, \tilde{t})}{\partial \tilde{t}^{2}} \vec{k} \times\left[P_{1} \vec{b}_{1}+P_{2} \vec{b}_{2}\right]=$ aceleração angular do motor.

$\left(\frac{\partial \theta(\tilde{x}, \tilde{t})}{\partial \tilde{t}}\right)^{2}\left[P_{1} \vec{b}_{1}+P_{2} \vec{b}_{2}\right]=$ aceleração centrípeta do motor.

$\frac{d^{2} \phi(\tilde{t})}{d \tilde{t}^{2}} \vec{k} \times\left[\vec{b}_{1} e \cos \phi+\vec{b}_{2} e \sin \phi\right]=$ aceleração angular da massa $m_{e}$.

$\left(\frac{d \phi}{d \tilde{t}}\right)^{2}\left[\vec{b}_{1} e \cos \phi+\vec{b}_{2} e \sin \phi\right]=$ aceleração centrípeta da massa $m_{e}$.

Os termos $\vec{d}_{1}$ e $\vec{d}_{2}$ representam as distâncias do ponto $A$ até o ponto $G$ medido ao longo de $\vec{b}_{1}$ e $\vec{b}_{2}$ como mostra a Figura 3.4.

Combinando Equação (3.1.19) e Equação (3.1.20) com Equação (3.1.18) juntamente com Equação (3.1.6), Equação (3.1.12) e Equação (3.1.13), e definindo: 


$$
\begin{aligned}
& A_{1}=\frac{E \beta^{3}}{\rho L} \frac{\partial^{2} u(x, t)}{\partial t^{2}} ; A_{2}=\frac{E \beta^{2}}{\rho L} \frac{\partial^{2} w(x, t)}{\partial t^{2}} ; A_{3}=\frac{E \beta^{2}}{\rho L^{2}} \frac{\partial^{3} w(x, t)}{\partial t^{2} \partial x} ; A_{4}=-\frac{E \beta^{3}}{\rho L^{2}}\left(\frac{\partial^{2} w(x, t)}{\partial t \partial x}\right)^{2} ; \\
& A_{5}=\frac{E \beta}{\rho L^{2}} \frac{d^{2} \phi(t)}{d t^{2}} ; A_{6}=-\frac{E \beta}{\rho L^{2}}\left(\frac{d \phi}{d t}\right)^{2} ; \vec{r}_{1}=\left[d_{1} \vec{b}_{1}+d_{2} \vec{b}_{2}\right] ; \vec{r}_{2}=\left[P_{1} \vec{b}_{1}+P_{2} \vec{b}_{2}\right] ; \\
& \vec{r}_{3}=\left\lfloor\vec{b}_{1} e \cos \phi+\vec{b}_{2} e \sin \phi\right]
\end{aligned}
$$

reescreve-se a Equação (3.1.19) e Equação (3.1.20) como segue.

$$
\begin{aligned}
& \vec{a}_{G}=A_{1} \vec{i}+A_{2} \vec{j}+A_{3}\left\lfloor\vec{k} \times \vec{r}_{1}\right\rfloor+A_{4} \vec{r}_{1} \\
& \vec{a}_{e}=\vec{a}_{G}+A_{3}\left(\vec{k} \times \vec{r}_{2}\right)+A_{4} \vec{r}_{2}+A_{5}\left(\vec{k} \times \vec{r}_{3}\right)+A_{6} \vec{r}_{3}
\end{aligned}
$$

Substituindo as expressões acima na Equação (3.1.18) obtém-se a expressão para $\tilde{N}$.

$$
\begin{aligned}
& \tilde{N}=e R_{1} m_{e}\left\{\frac{d^{2} \phi(t)}{d t^{2}}\left[\beta \cos \phi \frac{\partial w}{\partial x}+\sin \phi\right]-\left(\frac{d \phi(t)}{d t^{2}}\right)^{2}\left[\cos \phi+\beta \sin \phi \frac{\partial w}{\partial x}\right]\right\} \\
& +R_{2} \frac{\partial^{3} w(x, t)}{\partial x \partial t^{2}}\left\{M d_{2}+m_{e} L P_{2}\right\}+O\left(\beta^{4}\right)
\end{aligned}
$$

onde $M=m_{R B}+m_{e}$ e por conveniência define-se as variáveis adimensionais.

$$
R_{1}=\frac{\beta E}{\rho L^{2}} ; R_{2}=\frac{\beta^{2} E}{\rho L} ;
$$

Substituindo a Equação (3.1.6), Equação (3.1.9), Equação (3.1.11) e Equação (3.1.12) utilizando a Equação (3.1.13) e Equação (3.1.21) na Equação (3.1.2) têm-se.

$$
\begin{aligned}
& \frac{\beta}{L} \frac{\partial}{\partial x}\left(\tilde{N} \frac{\partial w}{\partial x}\right)-\beta \alpha \frac{\partial^{4} w}{\partial x^{4}}+\beta^{3} \alpha\left\{\frac{3}{2} \frac{\partial^{2}}{\partial x^{2}}\left(\frac{\partial w}{\partial x}\right)^{2}+\frac{1}{2} \frac{\partial}{\partial x}\left[\frac{\partial^{3} w}{\partial x^{3}}\left(\frac{\partial w}{\partial x}\right)^{2}\right]\right\} \\
& -\beta^{2} E A \frac{\partial^{2} w}{\partial t^{2}}=0
\end{aligned}
$$

onde $\alpha=\frac{E I}{L^{3}}$.

A Equação (3.1.23) é a equação da viga com $\tilde{N}$ dado pela Equação (3.1.21). A equação do movimento para o motor é dado por (Kononenko, 1969).

$$
I_{e}\left[\frac{d^{2} \phi}{d t^{2}}+\left.\frac{\partial^{3} w(x, t)}{\partial t^{2} \partial x}\right|_{x=1}\right]=T-D
$$


Sendo que cada termo da Equação (3.1.24) é descrito como abaixo.

$I_{e} \frac{d^{2} \phi}{d t^{2}}=$ Momento de Inércia da massa rotativa;

$I_{e} \frac{\partial^{3} w(x, t)}{\partial t^{2} \partial x}=$ Termo de acoplamento do elemento infinitesimal da viga com o motor;

$T=$ Torque do motor;

$D=$ Atrito aerodinâmico.

$\mathrm{Na}$ seção que segue, calculam-se as condições de contorno do sistema vigamotor.

\subsection{Obtenção das Condições de Contorno}

A Equação (3.1.23) é uma equação diferencial parcial de quarta ordem, então esta equação terá quatro condições de contorno. As duas primeiras são as condições que determinam o engaste da viga, como segue. O desenvolvimento dos cálculos desta seção encontra-se no Apêndice B.

$$
\left\{\begin{array}{l}
w(0, t)=0 \\
\left.\frac{\partial w(0, t)}{\partial x}\right|_{x=0}=0
\end{array}\right.
$$

A equação que determina a terceira condição de contorno, devido à ação das forças no sistema, é escrita como segue.

$$
\tilde{N} \sin \theta+\tilde{V} \cos \theta=\left[m_{R B} \vec{a}_{G}+m_{e} \vec{a}_{e}\right] \cdot \vec{j}
$$

Substituindo a Equação (3.1.6), Equação (3.1.9), Equação (3.1.12), Equação (3.1.13), Equação (3.1.19), Equação (3.1.20) e Equação (3.1.21) na equação acima, têm-se.

$$
\begin{aligned}
& {\left[\frac{\beta E I}{L^{2}}\left\{\frac{\partial^{3} w}{\partial x^{3}}-\beta^{2} \frac{3}{2}\left(\frac{\partial w}{\partial x}\right)^{2}\right\}\left(1-\frac{\beta^{2}}{2}\left(\frac{\partial w}{\partial x}\right)^{2}\right)\right]-\left[e R _ { 1 } m _ { e } \left\{\frac{d^{2} \phi}{d t^{2}}\left(\beta \cos \phi \frac{\partial w}{\partial x}+\sin \phi\right)-\right.\right.} \\
& \left.\left.\left(\frac{d \phi}{d t}\right)^{2}\left[\cos \phi+\beta \sin \phi \frac{\partial w}{\partial x}\right]\right\}+R_{2} \frac{\partial^{3} w}{\partial x \partial t^{2}}\left\{M d_{2}+m_{e} L P_{2}\right\}\right] \beta \frac{\partial w}{\partial x}-\frac{\beta^{3}}{2}\left(\frac{\partial w}{\partial x}\right)^{3}= \\
& {\left[m_{R B} \vec{a}_{G}+m_{e} \vec{a}_{e}\right] \cdot j}
\end{aligned}
$$


Somando os momentos em torno do centro de massa $m_{R B}$ e da massa desbalanceada $m_{e}$, como mostrado na Figura 3.3 e Figura 3.4, tem-se a quarta condição de contorno em termos das variáveis angulares como segue.

$$
\tilde{V}(\tilde{L}, \tilde{t}) d_{1}-\tilde{N}(\tilde{L}, \tilde{t}) d_{2}-\tilde{M}(\tilde{L}, \tilde{t})=\left(I_{R B}+I_{e}\right) \frac{\partial^{2} \theta(\tilde{L}, \tilde{t})}{\partial \tilde{t}^{2}}+I_{e} \frac{d^{2} \phi}{d t^{2}}
$$

onde

$\frac{\partial^{2} \theta(\tilde{L}, \tilde{t})}{\partial \tilde{t}^{2}}=$ aceleração angular da viga;

e

$$
\frac{d^{2} \phi}{d t^{2}}=\text { aceleração angular do motor. }
$$

Substituindo (3.1.6), (3.1.9), (3.1.14) e (3.1.21) em (3.2.3) tem-se.

$$
\begin{aligned}
& \left(-\frac{\beta E I}{L^{2}}\left[\frac{\partial^{3} w}{\partial x^{3}}-\beta^{2} \frac{3}{2}\left(\frac{\partial w}{\partial x}\right)^{2}\right]\right) d_{1}-\left(e R _ { 1 } m _ { e } \left\{\frac{d^{2} \phi}{d t^{2}}\left[\beta \cos \phi \frac{\partial w}{\partial x}+\sin \phi\right]-\right.\right. \\
& \left.\left.\left(\frac{d \phi}{d t}\right)^{2}\left[\cos \phi+\beta \sin \phi \frac{\partial w}{\partial x}\right]\right\}+R_{2} \frac{\partial^{3} w}{\partial x \partial t^{2}}\left\{M d_{2}+m_{e} L P_{2}\right\}\right) d_{2}-\frac{E I}{L} \beta\left[\frac{\partial^{2} w}{\partial x^{2}}-\beta^{2} \frac{3}{2}\left(\frac{\partial w}{\partial x}\right)^{2}\right]= \\
& \left(I_{R B}+I_{e}\right) \frac{\partial^{2}}{\partial t^{2}}\left(\beta \frac{\partial w}{\partial x}-\frac{\beta^{3}}{2}\left(\frac{\partial w}{\partial x}\right)^{3}\right)+I_{e} \frac{d^{2} \phi}{d t^{2}}
\end{aligned}
$$

Na seção que segue a Equação (3.1.23) será discretizada utilizando o método dos modos assumidos

\subsection{Análise Numérica das Equações do Movimento}

Para a resolução do problema em discussão escolheu-se o método dos modos assumidos (Meirovitch, 1970). Tal método consiste na expansão da solução da Equação (3.1.23) em uma base completa no espaço de funções.

$$
w(x, t)=\sum_{i} q_{i}(t) \Psi_{i}(x)
$$


Onde $q_{i}(t)$ são as coordenadas generalizadas dependentes do tempo e os modos $\Psi_{i}(x)$ são os elementos da base. Uma escolha conveniente para a base é dado pelo conjunto de soluções do problema livre $(\phi=0)$. Tal base, cujos cálculos são detalhados no Apêndice A, é dada por (Nayfeh, 1973, Nayfeh e Mook, 1979).

$$
\Psi_{i}(x)=\cosh \left(\lambda_{i} x\right)-\cos \left(\lambda_{i} x\right)+\gamma\left(\sinh \left(\lambda_{i} x\right)-\sin \left(\lambda_{i} x\right)\right)
$$

onde

$$
\lambda_{i}=\sqrt{w} .
$$

$\mathrm{e}$

$$
\gamma=-\frac{\lambda_{i}\left(\sinh \left(\lambda_{i} x\right)\left\{\lambda_{i}^{2}+\lambda_{i}^{4} R_{1} C_{1}\right\}\right)+C_{1}\left(\cosh \left(\lambda_{i} x\right)-\cos \left(\lambda_{i} x\right)\right)}{\lambda_{i}\left(\cosh \left(\lambda_{i} x\right)-\cos \left(\lambda_{i} x\right)\right)\left\{\lambda_{i}^{4} R_{1} C_{1}+\lambda^{3}\left(\cosh \left(\lambda_{i} x\right)+\cos \left(\lambda_{i} x\right)\right)\right\}+C_{1}\left(\sinh \left(\lambda_{i} x\right)-\sin \left(\lambda_{i} x\right)\right)} .
$$

sendo $C_{1}=M / \rho L A$.

Substituindo a Equação (3.3.1) na Equação (3.1.23) obtém-se o sistema de equações diferenciais acoplados para os modos $q_{i}(t)$.

$$
\begin{aligned}
& \frac{\beta}{L} \frac{d}{d x}\left(\tilde{N} \sum_{i} \Psi_{i}^{\prime} q_{i}\right)-\beta \alpha\left(\sum_{i} \Psi_{i}^{\prime \prime \prime \prime} q_{i}\right)+\beta^{3} \alpha\left\{\frac{3}{2} \frac{d^{2}}{d x^{2}}\left[\left(\sum_{i} \Psi_{i}^{\prime} q_{i}\right)\left(\sum_{j} \Psi_{j}^{\prime} q_{j}\right)\right]+\right. \\
& \left.\frac{1}{2} \frac{d}{d x}\left[\left(\sum_{i} \Psi_{i}^{\prime \prime \prime} q_{i}\right)\left\{\left(\sum_{p} \Psi_{p}^{\prime} q_{p}\right)\left(\sum_{v} \Psi_{v}^{\prime} q_{v}\right)\right\}\right]\right\}-\beta^{2} E A\left(\sum_{i} \Psi_{i} \ddot{q}_{i}\right)=0
\end{aligned}
$$

Desenvolvendo as derivadas dos termos da Equação (3.3.2) obtemos:

Primeiro termo da Equação (3.3.2):

$$
\frac{\beta}{L} \frac{d}{d x}\left(\tilde{N} \sum_{i} \Psi_{i}^{\prime} q_{i}\right)=\frac{\beta}{L} \frac{d \tilde{N}}{d x}\left(\sum_{i} \Psi_{i}^{\prime} q_{i}\right)+\tilde{N}\left(\sum_{i} \Psi_{i}^{\prime \prime} q_{i}\right)
$$

onde

$$
\begin{aligned}
& \frac{d \tilde{N}}{d x}=e R_{1} m_{e}\left\{\frac{d^{2} \phi(t)}{d t^{2}}\left[\cos \phi \beta\left(\sum_{i} \Psi_{i}^{\prime \prime} q_{i}\right)\right]-\left(\frac{d \phi(t)}{d t^{2}}\right)^{2}\left[\sin \phi \beta\left(\sum_{i} \Psi_{i}^{\prime \prime} q_{i}\right)\right]\right\} \\
& +R_{2}\left(\sum_{i} \Psi_{i}^{\prime \prime} \ddot{q}_{i}\right)\left\{M d_{2}+m_{e} L P_{2}\right\}+\mathrm{O}\left(\beta^{3}\right)
\end{aligned}
$$


Desenvolvendo o terceiro termo da Equação (3.3.2), obtemos:

$$
\frac{3}{2} \frac{d^{2}}{d x^{2}}\left[\left(\sum_{i} \Psi_{i}^{\prime} q_{i}\right)\left(\sum_{j} \Psi_{j}^{\prime} q_{j}\right)\right]=\frac{3}{2} \sum_{i, j}\left(\Psi_{i}^{\prime \prime \prime} \Psi_{j}^{\prime}+2 \Psi_{i}^{\prime \prime} \Psi_{j}^{\prime \prime}+\Psi_{i}^{\prime} \Psi_{j}^{\prime \prime \prime}\right) q_{i} q_{j}
$$

Desenvolvendo o quarto termo da Equação (3.3.2) como mostrado abaixo:

$$
\begin{aligned}
& \frac{d}{d x}\left[\left(\sum_{i} \Psi_{i}^{\prime \prime \prime} q_{i}\right)\left\{\left(\sum_{p} \Psi_{p}^{\prime} q_{p}\right)\left(\sum_{v} \Psi_{v}^{\prime} q_{v}\right)\right\}\right]= \\
& \sum_{i, p, v}\left(\Psi_{i}^{\prime \prime \prime} \Psi_{p}^{\prime} \Psi_{v}^{\prime}+\Psi_{i}^{\prime \prime \prime} \Psi_{p}^{\prime \prime} \Psi_{v}^{\prime}+\Psi_{i}^{\prime \prime \prime} \Psi_{p}^{\prime} \Psi_{v}^{\prime \prime}\right) q_{i} q_{p} q_{v}
\end{aligned}
$$

Substituindo a Equação (3.3.2a), Equação (3.3.2b) e Equação (3.3.2c) na Equação (3.3.2), temos:

$$
\begin{aligned}
& \beta\left(\frac{d \tilde{N}}{d x} \sum_{i} \Psi_{i}^{\prime} q_{i}\right)+\beta \tilde{N}\left(\sum_{i} \Psi_{i}^{\prime \prime} q_{i}\right)+\beta \alpha\left(\sum_{i} \Psi_{i}^{\prime \prime \prime} q_{i}\right)- \\
& \beta^{3} \alpha\left\{\frac{3}{2} \sum_{i, j}\left(\Psi_{i}^{\prime \prime \prime} \Psi_{j}^{\prime}+2 \Psi_{i}^{\prime \prime} \Psi_{j}^{\prime \prime}+\Psi_{i}^{\prime} \Psi_{j}^{\prime \prime \prime}\right) q_{i} q_{j}+\right. \\
& \left.\sum_{i, p, v}\left(\Psi_{i}^{\prime \prime \prime '} \Psi_{p}^{\prime} \Psi_{v}^{\prime}+\Psi_{i}^{\prime \prime \prime} \Psi_{p}^{\prime \prime} \Psi_{v}^{\prime}+\Psi_{i}^{\prime \prime \prime} \Psi_{p}^{\prime} \Psi_{v}^{\prime \prime}\right) q_{i} q_{p} q_{v}\right\}- \\
& \beta^{2} E A\left(\sum_{i} \Psi_{i} \ddot{q}_{i}\right)=0
\end{aligned}
$$

Multiplicando a Equação (3.3.2d) por $\Psi_{z}(x)$ e integrando entre 0 e 1 ao longo de todo o comprimento da viga, e considerando o fato de que o conjunto $\left\{\Psi_{i}(x)\right\}$ forma uma base no espaço de funções, pois é solução de um problema de valor de contorno (Butkov, 1988, Arfken, 1995), obtém-se:

$$
\begin{aligned}
& \beta\left(\frac{d \tilde{N}}{d x} \sum_{i} \Psi_{i}^{\prime} q_{i} \Psi_{z}\right)+\beta\left(\tilde{N} \sum_{i} \Psi_{i}^{\prime \prime} q_{i} \Psi_{z}\right)+\beta \alpha\left(\sum_{i} \Psi_{i}^{\prime \prime \prime \prime} q_{i} \Psi_{z}\right)- \\
& \beta^{3} \alpha\left\{\frac{3}{2} \sum_{i, j}\left(\Psi_{i}^{\prime \prime \prime} \Psi_{j}^{\prime} \Psi_{z}+2 \Psi_{i}^{\prime \prime} \Psi_{j}^{\prime \prime} \Psi_{z}+\Psi_{i}^{\prime} \Psi_{j}^{\prime \prime \prime} \Psi_{z}\right) q_{i} q_{j}+\right. \\
& \left.\sum_{i, p, v}\left(\Psi_{i}^{\prime \prime \prime \prime} \Psi_{p}^{\prime} \Psi_{v}^{\prime} \Psi_{z}+\Psi_{i}^{\prime \prime \prime} \Psi_{p}^{\prime \prime} \Psi_{v}^{\prime} \Psi_{z}+\Psi_{i}^{\prime \prime \prime} \Psi_{p}^{\prime} \Psi_{v}^{\prime \prime} \Psi_{z}\right) q_{i} q_{p} q_{v}\right\}- \\
& \beta^{2} E A\left(\sum_{i} \Psi_{i} \ddot{q}_{i} \Psi_{z}\right)=0
\end{aligned}
$$


Integrando a Equação (3.3.2e) tem-se:

$$
\begin{aligned}
& \beta\left(\sum_{i} q_{i} \int_{0}^{1} \frac{d \tilde{N}}{d x} \Psi_{i}^{\prime} \Psi_{z} d x+\sum_{i} q_{i} \int_{0}^{1} \tilde{N} \Psi_{i}^{\prime \prime} q_{i} \Psi_{z} d x\right)+\beta \alpha \sum_{i} q_{i} \int_{0}^{1} \Psi_{i}^{\prime \prime \prime} \Psi_{z} d x- \\
& \beta^{3} \alpha\left\{\frac{3}{2} \sum_{i, j} q_{i} q_{j}\left(\int_{0}^{1} \Psi_{i}^{\prime \prime \prime} \Psi_{j}^{\prime} \Psi_{z} d x+2 \int_{0}^{1} \Psi_{i}^{\prime \prime} \Psi_{j}^{\prime \prime} \Psi_{z} d x+\int_{0}^{1} \Psi_{i}^{\prime} \Psi_{j}^{\prime \prime '} \Psi_{z} d x\right)+\right. \\
& \left.\sum_{i, p, v} q_{i} q_{p} q_{v}\left(\int_{0}^{1} \Psi_{i}^{\prime \prime \prime \prime} \Psi_{p}^{\prime} \Psi_{v}^{\prime} \Psi_{z} d x+\int_{0}^{1} \Psi_{i}^{\prime \prime \prime} \Psi_{p}^{\prime \prime} \Psi_{v}^{\prime} \Psi_{z} d x+\int_{0}^{1} \Psi_{i}^{\prime \prime \prime} \Psi_{p}^{\prime} \Psi_{v}^{\prime \prime} \Psi_{z} d x\right)\right\}- \\
& \beta^{2} E A \ddot{q}_{z}=0
\end{aligned}
$$

Desenvolvendo e organizando o termo da Equação (3.3.2f) dado por:

$$
\begin{aligned}
& \sum_{i} q_{i} \int_{0}^{1} \tilde{N} \Psi_{i}^{\prime \prime} q_{i} \Psi_{z} d x= \\
& e R_{1} m_{e}\left\{\frac{d^{2} \phi(t)}{d t^{2}} \cos \phi \beta \sum_{i} \sum_{c} q_{i} q_{c} \int_{0}^{1} \Psi_{i}^{\prime} \Psi_{c}^{\prime \prime} \Psi_{z} d x+\frac{d^{2} \phi(t)}{d t^{2}} \sin \phi \sum_{c} q_{c} \int_{0}^{1} \Psi_{c}^{\prime \prime} \Psi_{z} d x-\right. \\
& \left.\left(\frac{d \phi}{d t}\right)^{2} \cos \phi \sum_{c} q_{c} \int_{0}^{1} \Psi_{c}^{\prime \prime} \Psi_{z} d x+\left(\frac{d \phi}{d t}\right)^{2} \sin \phi \beta \sum_{i} \sum_{c} q_{i} q_{c} \int_{0}^{1} \Psi_{i}^{\prime} \Psi_{c}^{\prime \prime} \Psi_{z} d x\right\}+ \\
& +R_{2} \sum_{i} \sum_{c} \ddot{q}_{i} q_{c}\left\{M d_{2}+m_{e} L P_{2}\right\} \int_{0}^{1} \Psi_{i}^{\prime} \Psi_{c}^{\prime \prime} \Psi_{z} d x
\end{aligned}
$$

sendo

$\int_{0}^{1} \Psi_{i}^{\prime} \Psi_{c}^{\prime \prime} \Psi_{z} d x=G_{1}$

$\int_{0}^{1} \Psi_{c}^{\prime \prime} \Psi_{z} d x=G_{2}$

substituindo $G_{1}$ e $G_{2}$ na Equação (3.3.2g) como segue:

$$
\begin{aligned}
& e R_{1} m_{e}\left\{\frac{d^{2} \phi}{d t^{2}} \cos \phi \beta \sum_{i} \sum_{c} q_{i} q_{c}\left(G_{1}\right)+\frac{d^{2} \phi}{d t^{2}} \sin \phi \sum_{c} q_{c}\left(G_{2}\right)-\left(\frac{d \phi}{d t}\right)^{2} \cos \phi \sum_{c} q_{c}\left(G_{2}\right)+\right. \\
& \left.\left(\frac{d \phi}{d t}\right)^{2} \sin \phi \beta \sum_{i} \sum_{c} q_{i} q_{c}\left(G_{1}\right)\right\}+R_{2} \sum_{i} \sum_{c} \ddot{q}_{i} q_{c}\left\{M d_{2}+m_{e} L P_{2}\right\}\left(G_{1}\right)
\end{aligned}
$$


Desenvolvendo e organizando o termo da Equação (3.3.2f) dado por:

$\sum_{i} q_{i} \int_{0}^{1} \frac{d \tilde{N}}{d x} \Psi_{i}^{\prime} \Psi_{z} d x=$

$e R_{1} m_{e}\left\{\frac{d^{2} \phi}{d t^{2}} \cos \phi \beta \sum_{i} \sum_{c} q_{i} q_{c} \int_{0}^{1} \Psi_{i}^{\prime \prime} \Psi_{c}^{\prime} \Psi_{z} d x-\right.$

$\left.\left(\frac{d \phi}{d t}\right)^{2} \operatorname{sen} \phi \beta \sum_{i} \sum_{c} q_{i} q_{c} \int_{0}^{1} \Psi_{i}^{\prime \prime} \Psi_{c}^{\prime} \Psi_{z} d x\right\}+R_{2} \sum_{i} \sum_{c} \ddot{q}_{i} q_{c}\left\{M d_{2}+m_{e} L P_{2}\right\} \int_{0}^{1} \Psi_{i}^{\prime \prime} \Psi_{c}^{\prime} \Psi_{z} d x$

sendo

$\int_{0}^{1} \Psi_{i}^{\prime \prime} \Psi_{c}^{\prime} \Psi_{z} d x=G_{3}$

substituindo $G_{3}$ na Equação (3.3.2i) obtemos:

$$
\begin{aligned}
& e R_{1} m_{e}\left\{\frac{d^{2} \phi}{d t^{2}} \cos \phi \beta \sum_{i} \sum_{c} q_{i} q_{c}\left(G_{3}\right)-\left(\frac{d \phi}{d t}\right)^{2} \operatorname{sen} \phi \beta \sum_{i} \sum_{c} q_{i} q_{c}\left(G_{3}\right)\right\}+ \\
& R_{2} \sum_{i} \sum_{c} \ddot{q}_{i} q_{c}\left\{M d_{2}+m_{e} L P_{2}\right\}\left(G_{3}\right)
\end{aligned}
$$

Substituindo a Equação (3.3.2h) e a Equação (3.3.2j) na Equação (3.3.2f) temos:

$$
\begin{aligned}
& e R_{1} m_{e}\left\{\frac{d^{2} \phi}{d t^{2}} \cos \phi \beta^{2} \sum_{i} \sum_{c} q_{i} q_{c}\left(G_{3}\right)-\left(\frac{d \phi}{d t}\right)^{2} \operatorname{sen} \phi \beta^{2} \sum_{i} \sum_{c} q_{i} q_{c}\left(G_{3}\right)\right\}+ \\
& R_{2} \sum_{i} \sum_{c} \ddot{q}_{i} q_{c}\left\{M d_{2}+m_{e} L P_{2}\right\}\left(G_{3}\right)+e R_{1} m_{e}\left\{\frac{d^{2} \phi}{d t^{2}} \cos \phi \beta^{2} \sum_{i} \sum_{c} q_{i} q_{c}\left(G_{1}\right)+\right. \\
& \left.\frac{d^{2} \phi}{d t^{2}} \sin \phi \sum_{c} q_{c}\left(G_{2}\right)-\left(\frac{d \phi}{d t}\right)^{2} \cos \phi \sum_{c} q_{c}\left(G_{2}\right)+\left(\frac{d \phi}{d t}\right)^{2} \sin \phi \beta^{2} \sum_{i} \sum_{c} q_{i} q_{c}\left(G_{1}\right)\right\}+ \\
& R_{2} \sum_{i} \sum_{c} \ddot{q}_{i} q_{c}\left\{M d_{2}+m_{e} L P_{2}\right\}\left(G_{1}\right)+\beta \alpha \sum_{i} q_{i}\left(G_{4}\right)-\beta^{3} \alpha\left\{\frac{3}{2} \sum_{i, j} q_{i} q_{j}\left(G_{5}\right)+\sum_{i, p, v} q_{i} q_{p} q_{v}\left(G_{6}\right)\right\}- \\
& \beta^{2} E A \ddot{q}_{z}=0
\end{aligned}
$$

onde

$$
\begin{aligned}
G_{4} & =\int_{0}^{1} \Psi_{i}^{\prime \prime \prime} \Psi_{z} d x ; \quad G_{5}=\int_{0}^{1} \Psi_{i}^{\prime \prime \prime} \Psi_{j}^{\prime} \Psi_{z} d x+2 \int_{0}^{1} \Psi_{i}^{\prime \prime} \Psi_{j}^{\prime \prime} \Psi_{z} d x+\int_{0}^{1} \Psi_{i}^{\prime} \Psi_{j}^{\prime \prime \prime} \Psi_{z} d x ; \\
G_{6} & =\int_{0}^{1} \Psi_{i}^{\prime \prime \prime} \Psi_{p}^{\prime} \Psi_{v}^{\prime} \Psi_{z} d x+\int_{0}^{1} \Psi_{i}^{\prime \prime \prime} \Psi_{p}^{\prime \prime} \Psi_{v}^{\prime} \Psi_{z} d x+\int_{0}^{1} \Psi_{i}^{\prime \prime \prime} \Psi_{p}^{\prime} \Psi_{v}^{\prime \prime} \Psi_{z} d x .
\end{aligned}
$$


Finalmente tem-se equação da viga como mostrado abaixo:

$$
\begin{aligned}
& e R_{1} m_{e}\left\{\ddot{\phi} \cos \phi \beta^{2} \sum_{i, c} q_{i} q_{c}\left[G_{3}+G_{1}\right]-\dot{\phi}^{2} \operatorname{sen} \phi \beta^{2} \sum_{i, c} q_{i} q_{c}\left[G_{3}-G_{1}\right]+\right. \\
& \left.\sum_{c} q_{c}\left(G_{2}\right)\left[\ddot{\phi} \operatorname{sen} \phi-\dot{\phi}^{2} \cos \phi\right]\right\}+R_{2} \sum_{i, c} \ddot{q}_{i} q_{c}\left(M d_{2}+m_{e} L P_{2}\right)\left[G_{3}+G_{1}\right]+ \\
& \beta \alpha \sum_{i} q_{i}\left(G_{4}\right)-\beta^{3} \alpha\left\{\sum_{i, j} q_{i} q_{j}\left(G_{5}\right)+\sum_{i, p, v} q_{i} q_{p} q_{v}\left(G_{6}\right)\right\}-\beta^{2} E A \ddot{q}_{z}=0
\end{aligned}
$$

É importante salientar aqui que esta equação dita a dinâmica do sistema até ordem $\beta^{3}$, valendo para qualquer modo de vibração, exceto modos com energia muito alta, que violariam a condição de que o raio de curvatura da viga deva permanecer muito menor que o comprimento da mesma.

Substituindo a Equação (3.3.1) na Equação (3.1.24) do motor obtemos:

$$
I_{e}\left[\frac{d^{2} \phi}{d t^{2}}+\sum_{i} \Psi_{i}^{\prime} \ddot{q}_{i}\right]=T-D .
$$

No tópico que segue será apresentada a equação do sistema para o primeiro modo de vibrar do sistema descrito pela Equação (3.3.3).

\subsection{Análise do Primeiro Modo de Vibrar}

Equação da viga para o $1^{\circ}$ modo de vibrar é dado por:

$$
\begin{aligned}
& \ddot{q}\left(R_{2} q\left[M d_{2}+m_{e} L P_{2}\right]\left[G_{3}+G_{1}\right]-\beta^{2} E A\right)+q\left(\sigma G_{2} \ddot{\phi} \sin \phi-\dot{\phi}^{2} \sigma G_{2} \cos \phi+\beta \alpha G_{4}\right)+ \\
& q^{2}\left(\ddot{\phi} \sigma \beta^{2} \cos \phi\left[G_{3}+G_{1}\right]-\dot{\phi}^{2} \sigma \beta^{2} \sin \phi\left[G_{3}-G_{1}\right]-\beta^{3} \alpha G_{5}\right)-q^{3} \beta^{3} \alpha G_{6}=0 \\
& \text { onde } \sigma=\frac{e R_{1} m_{e}}{L}
\end{aligned}
$$

Equação do motor.

$$
I_{e} \ddot{\phi}+I_{e} \ddot{q} \Psi^{\prime}=T-D
$$

De forma a validar o modelo, apresenta-se uma comparação da Equação (3.4.1) com o sistema não ideal de Duffing encontrado na literatura. 


\subsubsection{Comparação com o Modelo de Duffing}

Reescreve-se a Equação (3.4.1) como segue.

$\ddot{q}(p q-\delta)+\eta q+\kappa q^{2}-\chi q^{3}=F_{1}$

onde $F_{1}$ é uma força externa (adicionando um shaker ao sistema) que não depende de $q$ (considera-se neste problema $F_{1}=0$ ) e $p, \delta, \eta, \kappa, \chi$ são funções obtidas diretamente da Equação (3.4.1).

O sistema não ideal e não amortecido de Duffing, na forma adimensional, é dado por (Zukovic, 2006 e Palacios, 2003).

$$
\ddot{q}+\omega^{2} q+p q^{3}=F_{2}
$$

Onde $F_{2}, \varpi$, e $p$ não dependem de $q$.

Comparando-se o lado esquerdo da Equação (3.4.3) com a Equação (3.4.4) observa-se que na Equação (3.4.3) os termos $\ddot{q}(p q)$ e $\kappa q^{2}$ não aparecem no modelo de Duffing, devido às seguintes considerações feitas na presente modelagem:

- A curvatura da viga e a sua deformação axial;

- O termo $\kappa q^{2}$ aparece, pois se considerou na expansão da Equação (3.1.5) até termos da ordem de $\beta^{3}$.

- O termo $\ddot{q}(p q)$ aparece, pois na Equação (3.1.21) se considerou termos até a ordem de $\beta^{3}$.

Desta forma pode-se afirmar que a Equação (3.4.4) está contida na Equação (3.4.3). Porém, os termos adicionais que aparecem na Equação (3.4.3) são justificados de acordo com as considerações feitas na modelagem apresentadas acima.

Na seção que segue, escreve-se a Equação (3.4.1) e Equação (3.4.2) na forma espaço de estado. 


\subsubsection{Sistema na Forma Espaço de Estado}

Reescrevendo a Equação (3.4.1).

$$
\ddot{q}\left(\alpha_{1}\right)+\ddot{\phi}\left(\alpha_{2}\right)-B+\ddot{\phi}\left(\alpha_{3}\right)-B_{1}-B_{2}=0
$$

onde

$$
\begin{aligned}
& \alpha_{1}=R_{2} q\left[M d_{2}+m_{e} L P_{2}\right]\left[G_{3}+G_{1}\right]-\beta^{2} E A ; \\
& \alpha_{2}=q \sigma G_{2} \sin \phi ; \\
& \alpha_{3}=q^{2} \sigma \beta^{2} \cos \phi\left[G_{3}+G_{1}\right] ; \\
& B=q\left(\dot{\phi}^{2} \sigma G_{2} \cos \phi+\beta \alpha G_{4}\right) ; \\
& B_{1}=q^{2}\left(\dot{\phi}^{2} \sigma \beta^{2} \sin \phi\left[G_{3}-G_{1}\right]-\beta^{3} \alpha G_{5}\right) ; \\
& B_{2}=q^{3} \beta^{3} \alpha G_{6} \\
& B_{3}=B+B_{1}+B_{2}
\end{aligned}
$$

Sendo que a Equação (3.4.5) pode ser escrita como segue, juntamente com a equação do motor.

$$
\begin{aligned}
& \ddot{q}\left(\alpha_{1}\right)+\ddot{\phi}\left(\alpha_{2}+\alpha_{3}\right)=B_{3} \\
& \ddot{q}\left(I_{e} \Psi^{\prime}\right)+\ddot{\phi}\left(I_{e}\right)=T-D
\end{aligned}
$$

Para introduzir a Equação (3.4.2) na Equação (3.4.5) deve-se escrever o sistema na forma matricial dado por.

$$
\left(\begin{array}{cc}
\alpha_{1} & \alpha_{2}+\alpha_{3} \\
I_{e} \Psi^{\prime} & I_{e}
\end{array}\right)\left(\begin{array}{l}
\ddot{q} \\
\ddot{\phi}
\end{array}\right)=\left(\begin{array}{c}
B_{3} \\
T-D
\end{array}\right)
$$

Organizando os termos da Equação (3.4.8) tem-se

$$
\left(\begin{array}{c}
\ddot{q} \\
\ddot{\phi}
\end{array}\right)=\left(\begin{array}{cc}
\alpha_{1} & \alpha_{2}+\alpha_{3} \\
I_{e} \Psi^{\prime} & I_{e}
\end{array}\right)^{-1}\left(\begin{array}{c}
B_{3} \\
T-D
\end{array}\right)
$$

Calcula-se a matriz inversa da Equação (3.4.9) obtém-se

$$
\left(\begin{array}{c}
\ddot{q} \\
\ddot{\phi}
\end{array}\right)=\left(\begin{array}{cc}
\frac{1}{\alpha_{1}-\left(\alpha_{2}+\alpha_{3}\right) \Psi^{\prime}} & \frac{-\left(\alpha_{2}+\alpha_{3}\right)}{I_{e} \alpha_{1}-I_{e}\left(\alpha_{2}+\alpha_{3}\right) \Psi^{\prime}} \\
\frac{-\Psi^{\prime}}{\alpha_{1}-\left(\alpha_{2}+\alpha_{3}\right) \Psi^{\prime}} & \frac{\alpha_{1}}{I_{e} \alpha_{1}-I_{e}\left(\alpha_{2}+\alpha_{3}\right) \Psi^{\prime}}
\end{array}\right)\left(\begin{array}{c}
B_{3} \\
T-D
\end{array}\right)
$$


Desenvolve-se o produto das matrizes da Equação (3.4.10) de forma a obter o sistema dado por.

$$
\left(\begin{array}{c}
\ddot{q} \\
\ddot{\phi}
\end{array}\right)=\frac{1}{\Delta}\left(\begin{array}{l}
B_{3}-\frac{\left(\alpha_{2}+\alpha_{3}\right)(T-D)}{I_{e}} \\
-B_{3}\left(\Psi^{\prime}\right)+\frac{\alpha_{1}(T-D)}{I_{e}}
\end{array}\right)
$$

onde

$$
\Delta=\alpha_{1}-\left(\alpha_{2}+\alpha_{3}\right) \Psi^{\prime}
$$

As variáveis de estado são escolhidas como abaixo.

$$
\begin{aligned}
& x_{1}=q ; x_{2}=\dot{q} ; \\
& x_{3}=\phi ; x_{4}=\dot{\phi}
\end{aligned}
$$

Substituem-se as variáveis de estado na Equação (3.4.11) como segue.

$$
\begin{aligned}
& \dot{x}_{1}=x_{2} \\
& \dot{x}_{2}=\frac{1}{\Delta}\left(B_{3}-\frac{\left(\alpha_{2}+\alpha_{3}\right)(T-D)}{I_{e}}\right) \\
& \dot{x}_{3}=x_{4} \\
& \dot{x}_{4}=\frac{1}{\Delta}\left(-B_{3}\left(\Psi^{\prime}\right)+\frac{\alpha_{1}(T-D)}{I_{e}}\right)
\end{aligned}
$$

Finalmente, as constantes da Equação (3.4.6) são substituídas na Equação (3.4.12), de forma a obter a equação do movimento do sistema representado na Figura 3.4, como segue.

$$
\begin{aligned}
\dot{x}_{1}= & x_{2} ; \\
\dot{x}_{2}= & \frac{1}{\left(R_{2} x_{1}[H]\left[G_{3}+G_{1}\right]-S\right)-\left(x_{1} Q \sin \left(x_{3}\right)+x_{1}^{2} Z \cos \left(x_{3}\right)\right) \Psi^{\prime}}\left[\left(x_{1}\left(x_{4}^{2} U \cos \left(x_{3}\right)+V\right)+\right.\right. \\
& \left.\left.x_{1}^{2}\left(x_{4}^{2} W \sin \left(x_{3}\right)-K\right)+x_{1}^{3} J\right)-\left(\frac{x_{1} Q \sin \left(x_{3}\right)+x_{1}^{2} Z \cos \left(x_{3}\right)}{I_{e}}\right)(T-D)\right] ; \\
\dot{x}_{3}= & x_{4} ; \\
\dot{x}_{4}= & \frac{-1}{\left(R_{2} x_{1}[H]\left[G_{3}+G_{1}\right]-S\right)-\left(x_{1} Q \sin \left(x_{3}\right)+x_{1}^{2} Z \cos \left(x_{3}\right)\right) \Psi^{\prime}}\left[\left(x_{1}\left(x_{4}^{2} U \cos \left(x_{3}\right)+V\right)+\right.\right. \\
& \left.\left.x_{1}^{2}\left(x_{4}^{2} W \sin \left(x_{3}\right)-K\right)+x_{1}^{3} J\right)\left(\Psi^{\prime}\right)+\left(\frac{\left(R_{2} x_{1}[H]\left[G_{3}+G_{1}\right]-S\right)(T-D)}{I_{e}}\right)\right]
\end{aligned}
$$


onde

$H=\left[M d_{2}+m_{e} L P_{2}\right]$;

$S=\beta^{2} E A$;

$U=\sigma G_{2}$

$V=\beta \alpha G_{4}$;

$W=\sigma \beta^{2}\left[G_{3}-G_{1}\right]$

$K=\beta^{3} \alpha G_{5}$;

$J=\beta^{3} \alpha G_{6} ;$

$Q=\sigma G_{2}$;

$Z=\sigma \beta^{2}\left[G_{3}+G_{1}\right]$.

No capítulo seguinte serão feitas as simulações numéricas do sistema representado pela Equação (3.4.13). 


\section{Capítulo 4}

\section{Resultados de Simulações Numéricas}

Os resultados apresentados neste capítulo baseiam-se nas simulações numéricas do sistema de equações descritas pela Equação (3.4.13) apresentada no Capítulo 3.

\subsection{Análise Dinâmica do Sistema: Rota para o Caos - Caso Ideal}

A análise dinâmica do sistema consiste em verificar como o sistema se comporta quando o parâmetro de controle é variado. O parâmetro de controle escolhido é o torque do motor $T(N \cdot m)$, pois é o torque fornecido pelo motor que controla a ocorrência de diferentes modos de oscilação do sistema.

Uma vez escolhido o valor do torque do motor que será variado, pretende-se observar, através de resultados de simulação numérica, para quais valores do parâmetro de controle o sistema perde estabilidade até alcançar ou não um regime caótico. Essas análises serão realizadas através da construção dos seguintes gráficos: Histórico no tempo, Plano de fase, FFT e expoentes de Lyapunov (Andrade, 2003) e o efeito Sommerfeld.

O programa utilizado nas simulações numéricas foi o MATLAB ${ }^{\circledR}$ 8.0. Os valores dos parâmetros usados nas simulações são mostrados na tabela como segue.

Tabela 4.1 - Parâmetros do sistema.

\begin{tabular}{|lllll|ll|}
\hline$M=0.5 \mathrm{~kg}$ & pg. 27 & $\alpha=12.8 \mathrm{~N} \cdot m$ & pg. 27 & $G 5=-0.0348$ & pg. 33 \\
\hline$m_{e}=3 \cdot 10^{-2} \mathrm{~kg}$ & pg. 25 & $C_{1}=3.6$ & pg. 30 & $G 6=-0.5153$ & pg. 33 \\
\hline$\rho \cdot A=0.89 \mathrm{~kg} / m$ & & $\sigma=7.5 \cdot 10^{5}$ & pg. 34 & $R_{1}=7.5 \cdot 10^{8}$ & pg. 27 \\
\hline$E \cdot I=1.01 \mathrm{Nm}^{2}$ & & $d_{2}=3 \cdot 10^{-2}$ & pg. 25 & $R_{2}=2 \cdot 10^{6}$ & pg. 27 \\
\hline$L=0.4 m$ & pg. 20 & $G 1=G 3=0.1697$ & pg. 32 & $\beta=3 \cdot 10^{-3}$ & pg. 23 \\
\hline$P_{2}=1 \cdot 10^{-2} m$ & pg. 25 & $G 2=0.1995$ & pg. 32 & & \\
\hline$r=4 \cdot 10^{-2} m$ & pg. 56 & $G 4=-0.2535$ & pg. 33 & & \\
\hline
\end{tabular}

A seguir são apresentados os resultados de simulação numérica. 
O histórico no tempo, plano de fase e FFT para $T=0.5$ são apresentados na Figura 4.1 como abaixo.

(a)

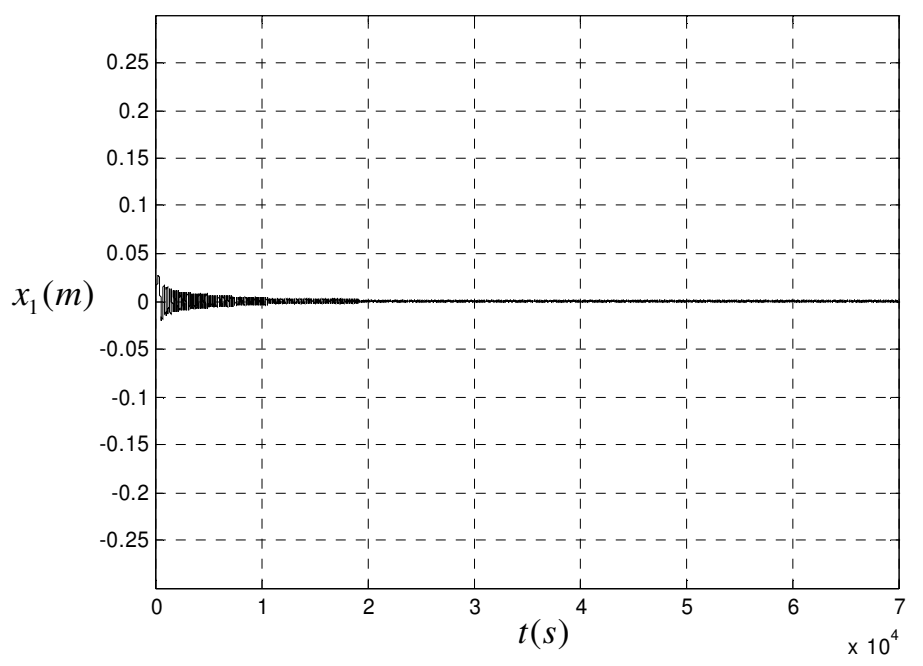

(b)

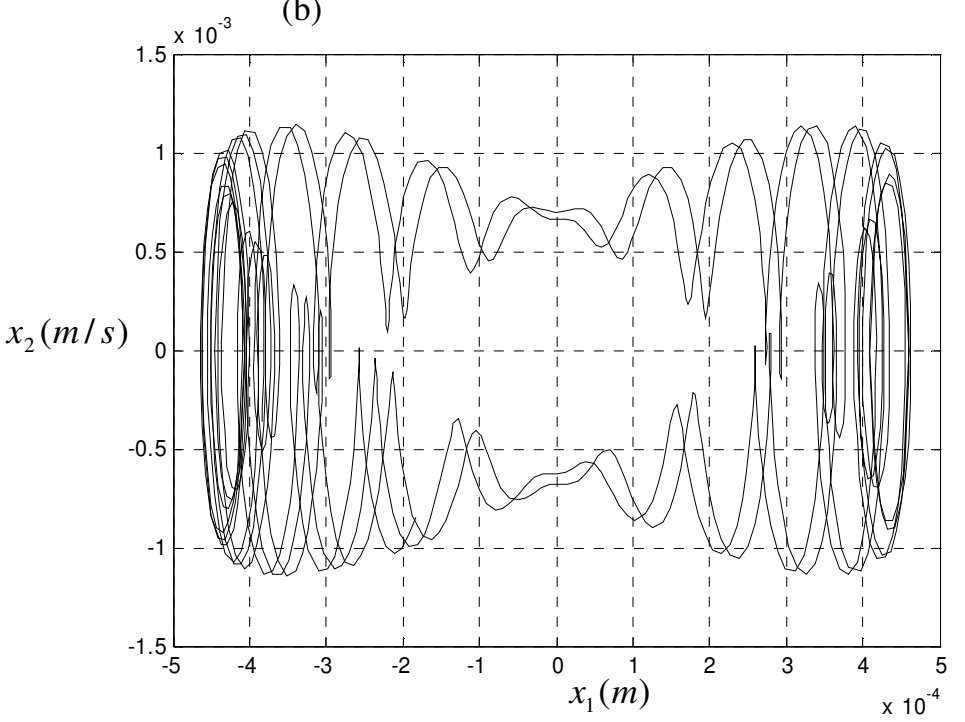

(c)

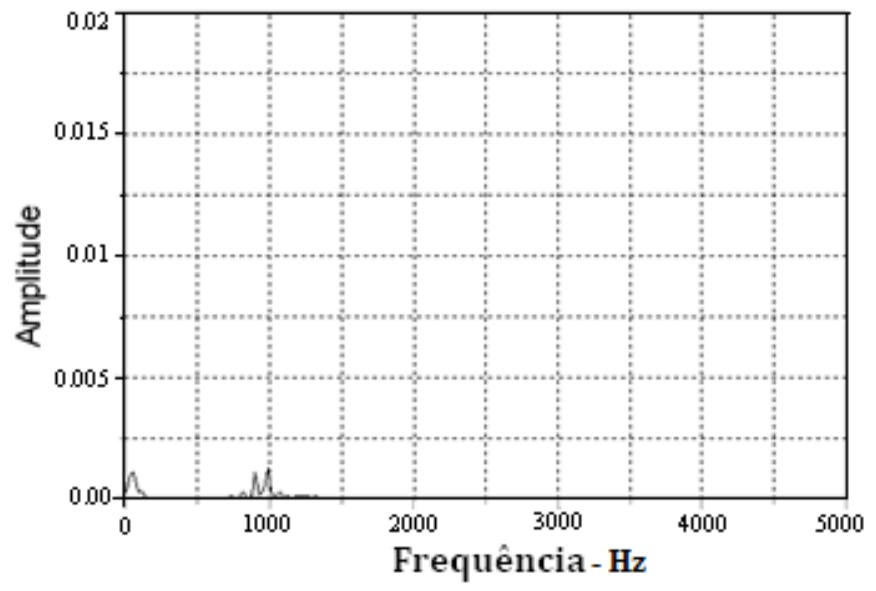

Figura 4.1 - (a) Histórico no tempo. (b) Plano de fase. (c) FFT. 
A Figura 4.1 mostra que o sistema tem um comportamento regular, pois o sistema apresenta na Figura 4.1(a) um histórico no tempo com oscilações de baixa amplitude em torno do ponto zero. Na Figura 4.1(b) observa-se um plano de fase com pequenas oscilações também ao redor do ponto zero e na Figura 4.1(c) a FFT mostra três pequenos picos, o que pode caracterizar que o sistema é periódico e de períodos três.

A análise que caracteriza o comportamento caótico ou não do sistema, para este parâmetro, é feita através do cálculo dos expoentes de Lyapunov como segue.
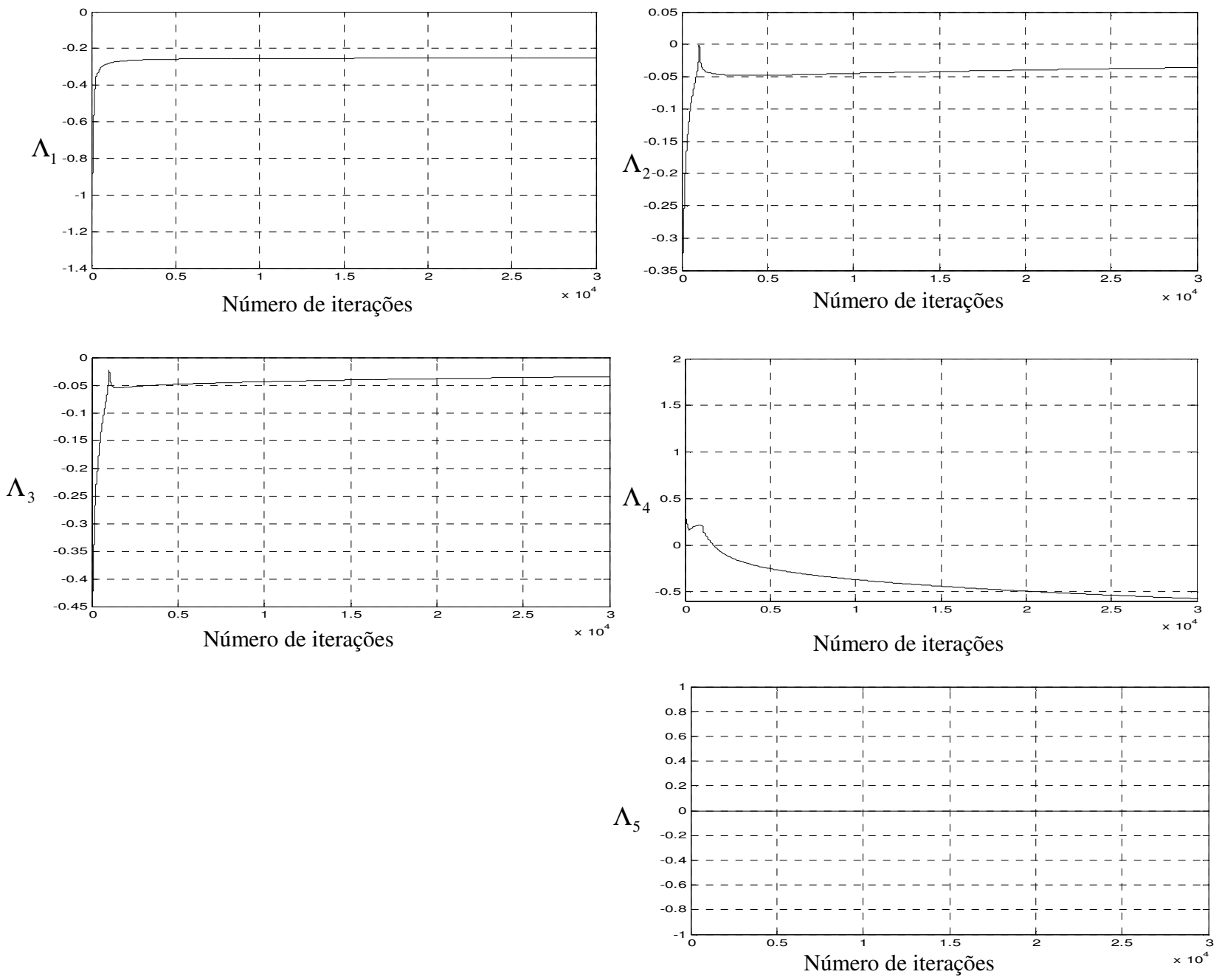

Figura 4.2 - Evolução dos expoentes de Lyapunov.

A Figura 4.2 mostra o comportamento dos expoentes de Lyapunov em função do número de iterações. Para o torque $T=0.5$ os valores dos expoentes de Lyapunov são: 
Tabela 4.2 - Expoentes de Lyapunov para o torque do motor $T=0.5$.

\begin{tabular}{|l|l|}
\hline $\begin{array}{c}\text { Expoentes de } \\
\text { Lyapunov }(\Lambda)\end{array}$ & Valores \\
\hline$\Lambda_{1}$ & -0.2528 \\
\hline$\Lambda_{2}$ & -0.0359 \\
\hline$\Lambda_{3}$ & -0.0345 \\
\hline$\Lambda_{4}$ & -0.5719 \\
\hline$\Lambda_{5}$ & 0 \\
\hline
\end{tabular}

Analisando a Tabela 4.2 pode-se afirmar que, para o torque $T=0.5$, o sistema apresenta um comportamento regular, pois os expoentes de Lyapunov são todos não positivos.

$\mathrm{Na}$ análise seguinte, apresenta-se o comportamento do sistema quando o valor do torque aumenta para $T=1.5$, como mostrado na Figura 4.3.

O gráfico histórico no tempo, plano de fase e FFT são mostrados como segue.

(a)

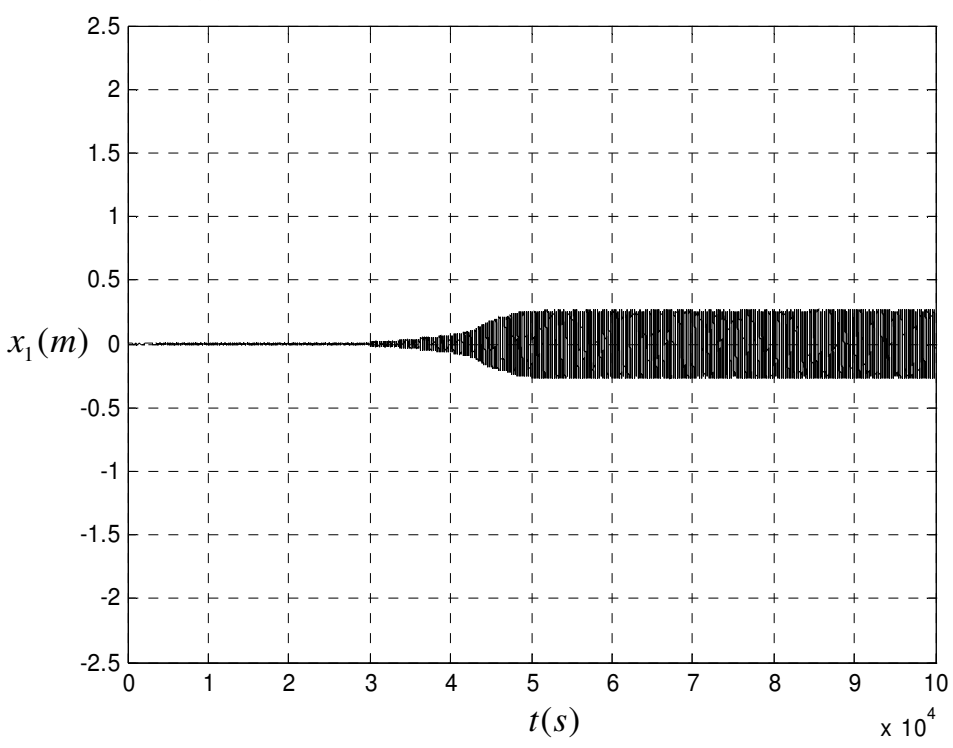


(b)

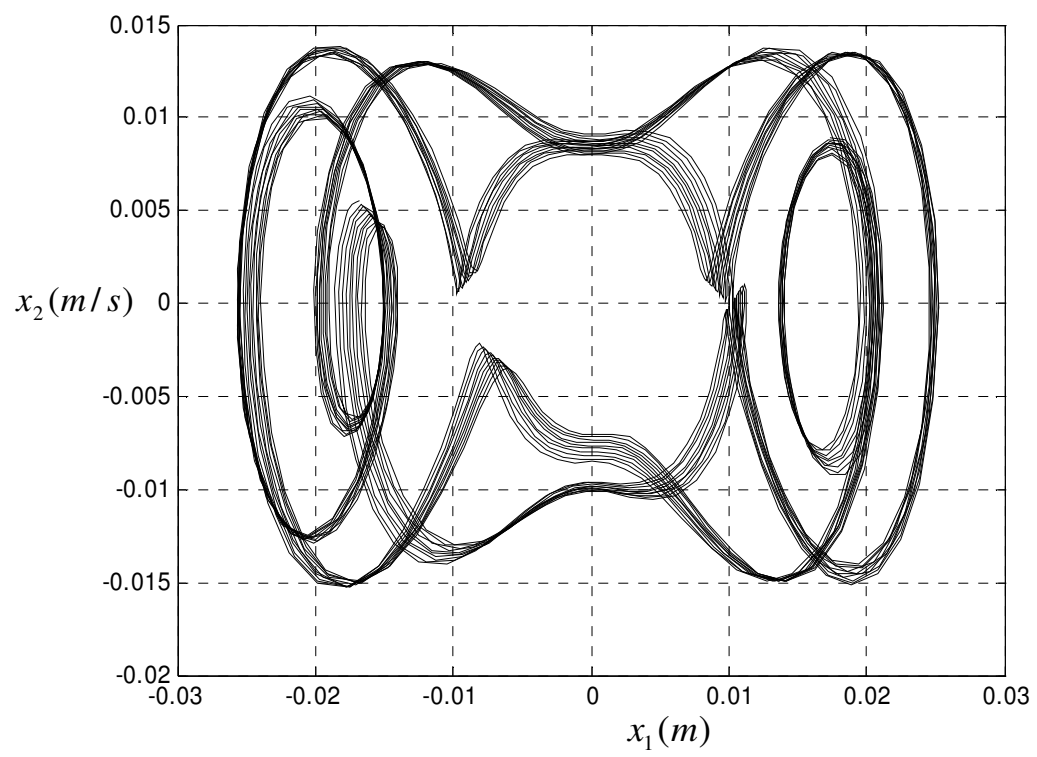

(c)

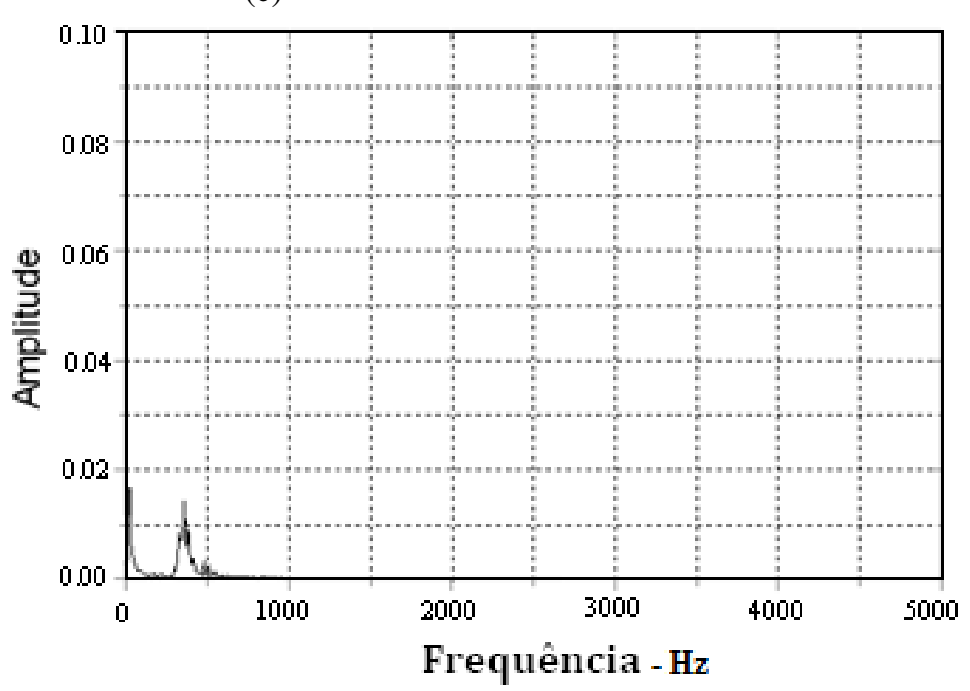

Figura 4.3 - (a) Histórico no tempo. (b) Plano de fase. (c) FFT.

A Figura 4.3 apresenta uma pequena alteração no movimento, porém ainda com características de um comportamento estável, pois na Figura 4.3(a) observa-se que o histórico no tempo apresentou um pequeno aumento de amplitude. Na Figura 4.3(b) o plano de fase apresentou um aumento nas oscilações, mas continua oscilando ao redor do ponto zero e na Figura 4.3(c) a FFT mostra dois picos bem definidos, o que pode caracterizar que o sistema é periódico e de períodos dois. 
$\mathrm{Na}$ análise seguinte, os expoentes de Lyapunov são calculados para confirmar a estabilidade do sistema.
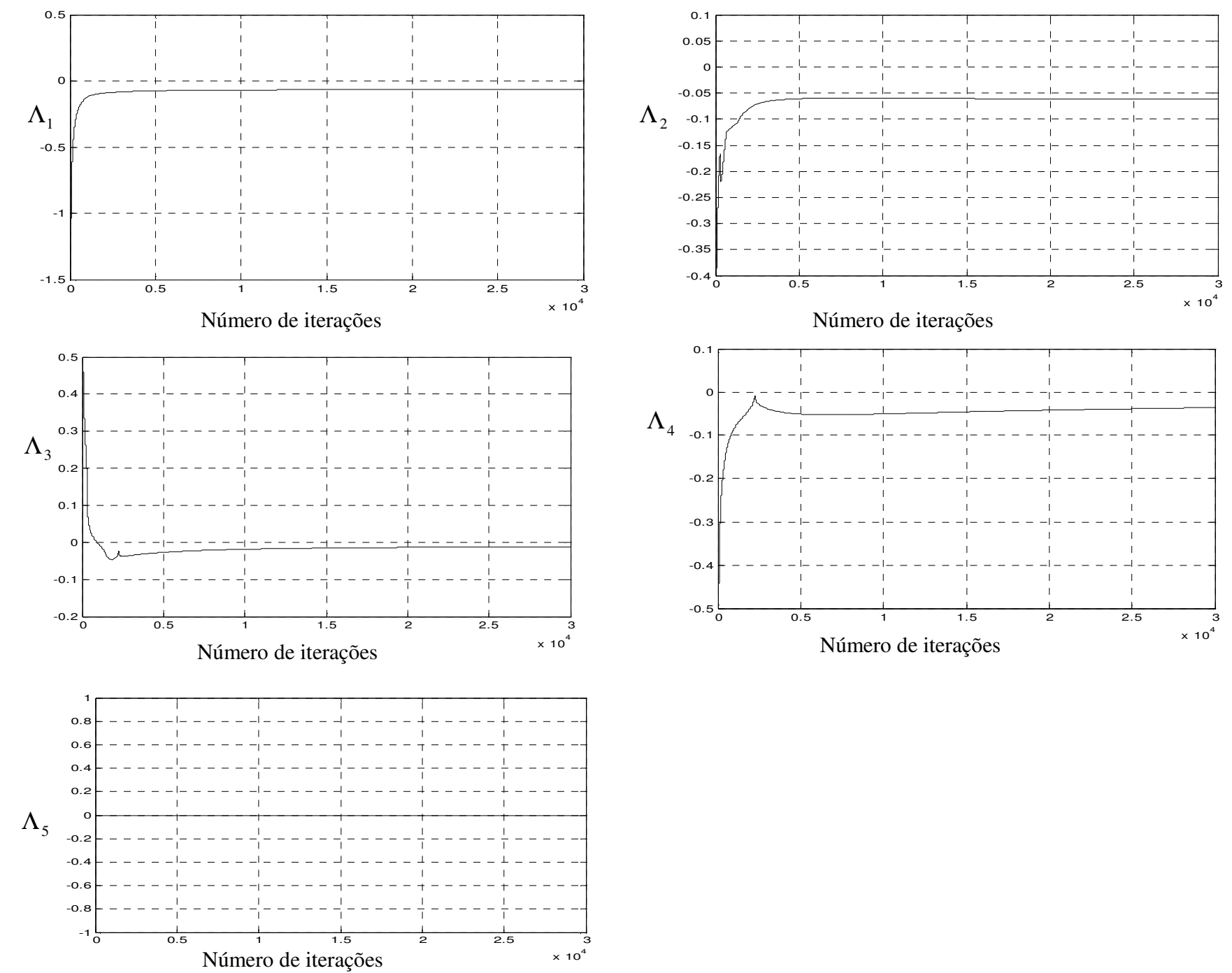

Figura 4.4 - Evolução dos expoentes de Lyapunov.

Para $T=1.5$, observa-se na Figura 4.4 que os valores dos expoentes de Lyapunov são:

Tabela 4.3 - Expoentes de Lyapunov para o torque do motor $T=1.5$.

\begin{tabular}{|l|l|}
\hline $\begin{array}{c}\text { Expoentes de } \\
\text { Lyapunov }(\Lambda)\end{array}$ & Valores \\
\hline$\Lambda_{1}$ & -0.0639 \\
\hline$\Lambda_{2}$ & -0.0623 \\
\hline$\Lambda_{3}$ & -0.0117 \\
\hline$\Lambda_{4}$ & -0.0359 \\
\hline$\Lambda_{5}$ & 0 \\
\hline
\end{tabular}


Desta forma, pode-se afirmar que o sistema apresenta um comportamento regular, pois todos os expoentes de Lyapunov são não positivos.

O comportamento dinâmico do sistema, quando o valor do torque aumenta para $T=2.5$, é apresentado na Figura 4.5.

Os gráficos, (a) histórico no tempo, (b) plano de fase e (c) FFT são mostrados como abaixo.
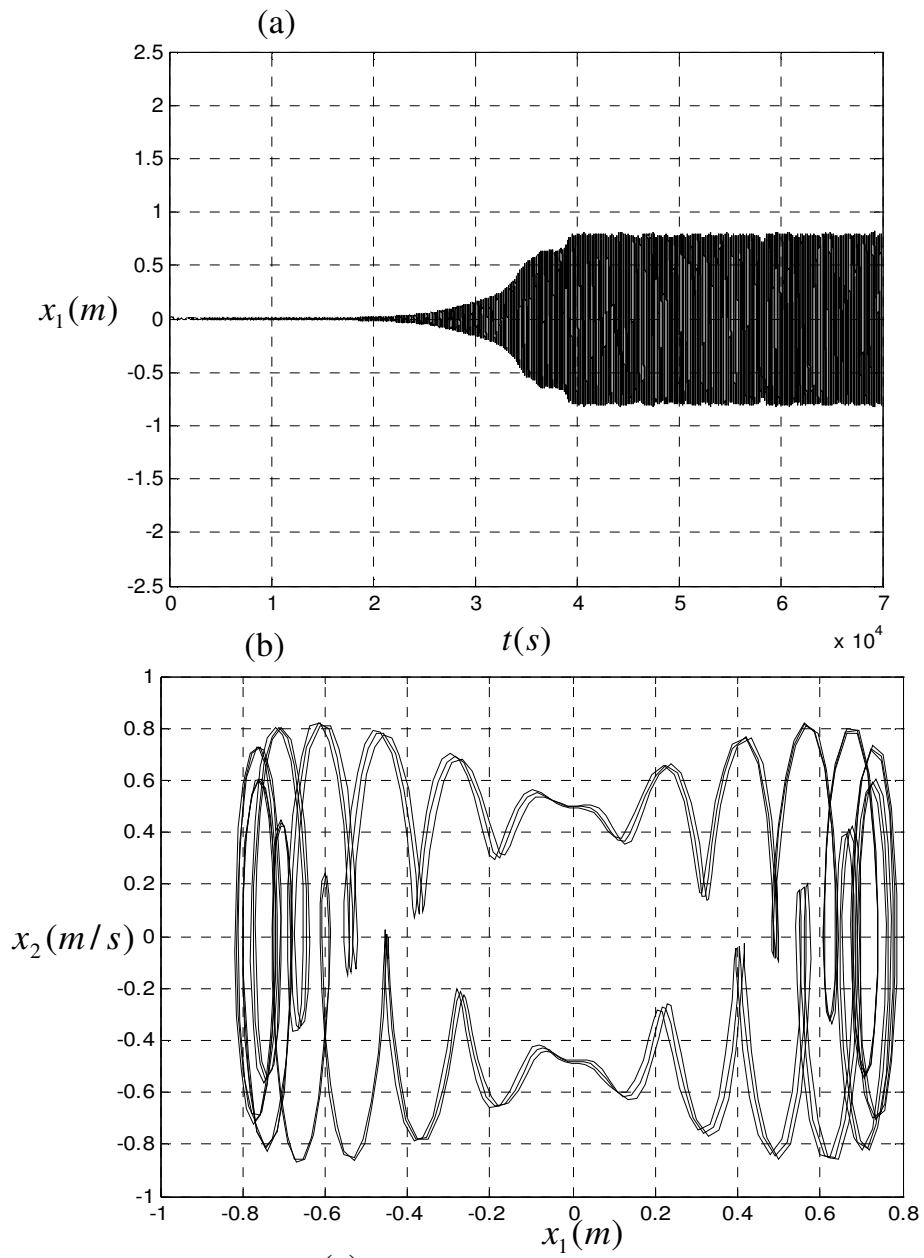

(c)

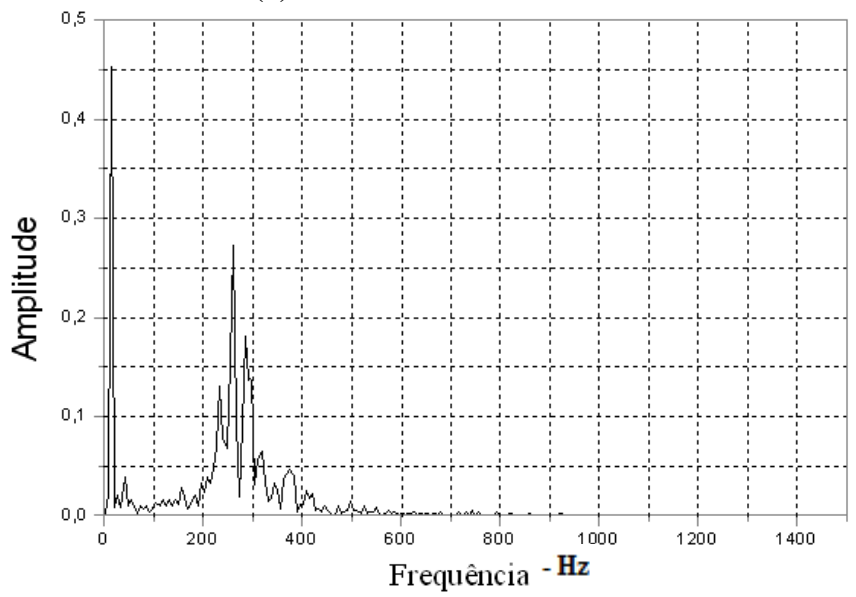

Figura 4.5 - (a) Histórico no tempo. (b) Plano de fase. (c) FFT. 
A Figura 4.5 mostra que o sistema apresenta muitas oscilações, podendo ou não ser característica de um comportamento caótico. A Figura 4.5(a) mostra que o aumento de amplitude nas oscilações do histórico no tempo continua, porém não mais próximo do ponto zero. O mesmo acontece na Figura 4.5(b), onde se observa que as amplitudes nas oscilações do ciclo limite tiveram um aumento significativo. Esse aumento na amplitude também é observado na Figura 4.5(c), onde a FFT apresentou um aumento no número de picos. Sendo assim, calculam-se os expoentes de Lyapunov para determinar se o sistema apresenta um comportamento caótico.

A evolução dos expoentes de Lyapunov é apresentada na Figura 4.6 como segue.
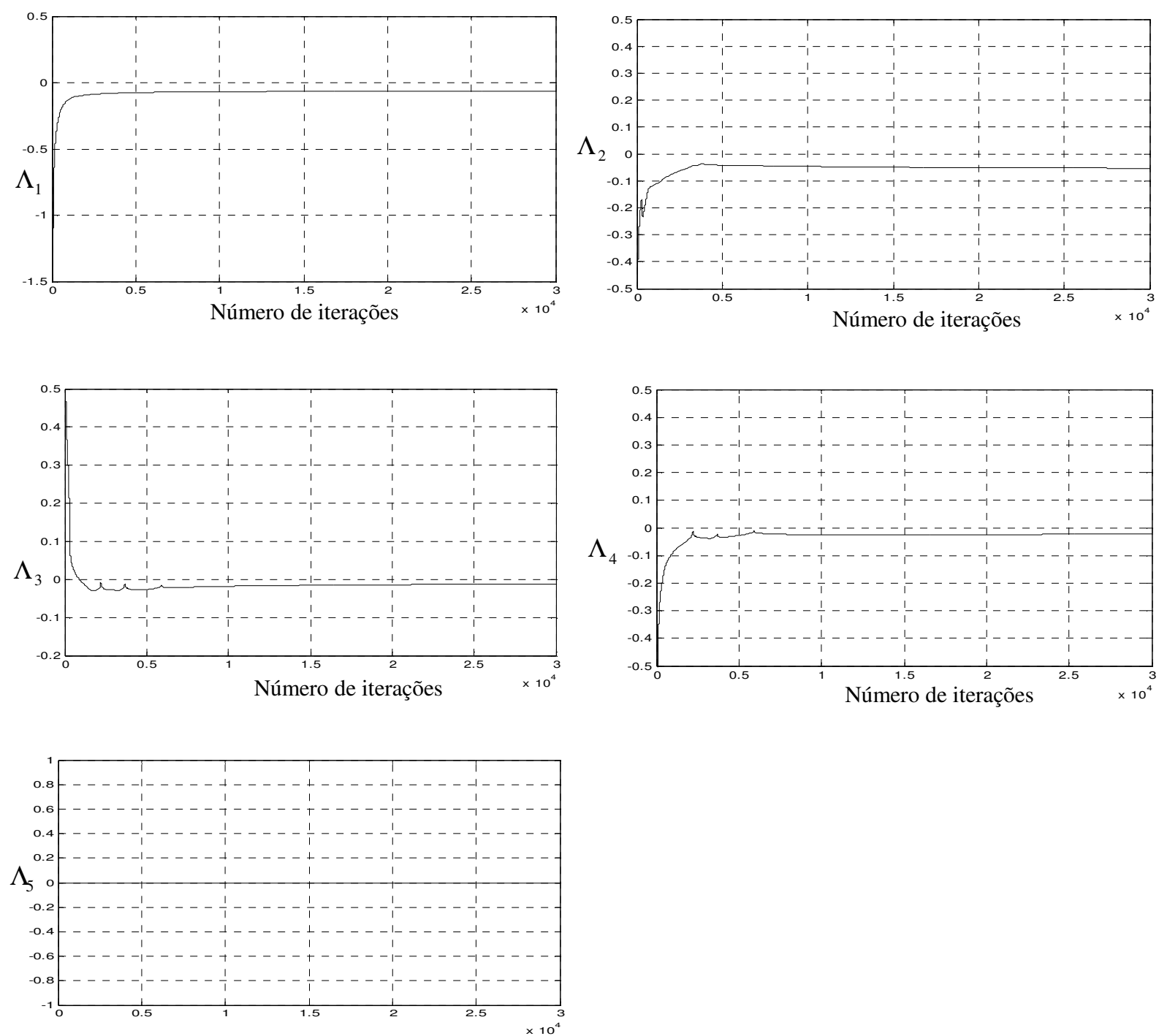

Número de iterações

Figura 4.6 - Evolução dos expoentes de Lyapunov. 
Para $T=2.5$ os valores dos expoentes de Lyapunov são:

Tabela 4.4 - Expoentes de Lyapunov para o torque do motor $T=2.5$.

\begin{tabular}{|l|l|}
\hline $\begin{array}{c}\text { Expoentes de } \\
\text { Lyapunov }(\Lambda)\end{array}$ & Valores \\
\hline$\Lambda_{1}$ & -0.0637 \\
\hline$\Lambda_{2}$ & -0.0529 \\
\hline$\Lambda_{3}$ & -0.0124 \\
\hline$\Lambda_{4}$ & -0.0219 \\
\hline$\Lambda_{5}$ & 0 \\
\hline
\end{tabular}

De acordo com os valores dos expoentes, conclui-se que o sistema apresenta um comportamento regular devido a todos os expoentes de Lyapunov serem não positivos.

$\mathrm{Na}$ análise que segue, o valor do torque é aumentado para $T=4.5$.

O histórico no tempo, plano de fase e FFT são apresentados na Figura 4.7 como segue.

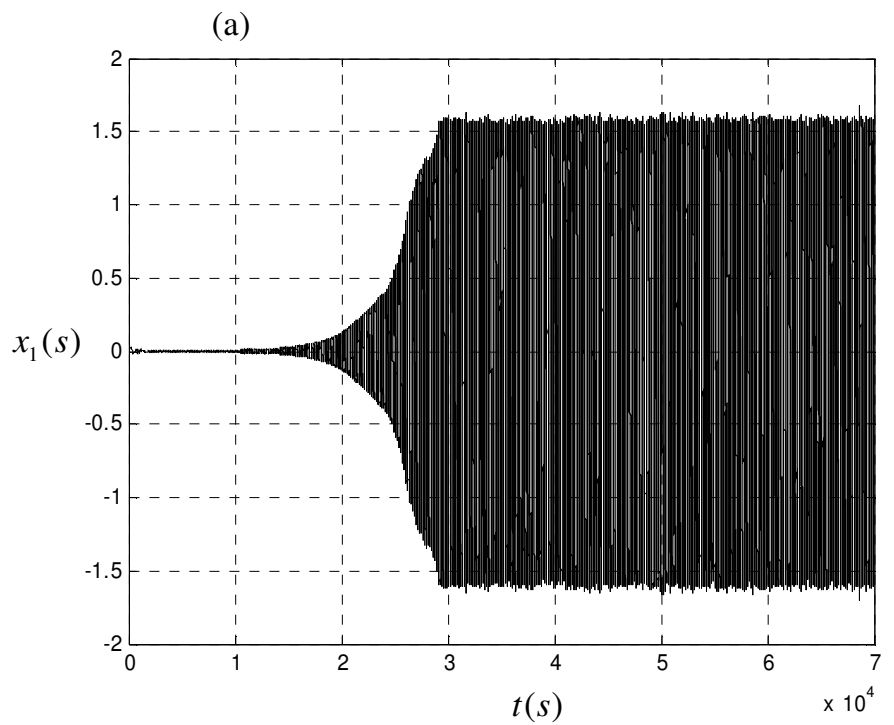


(b)

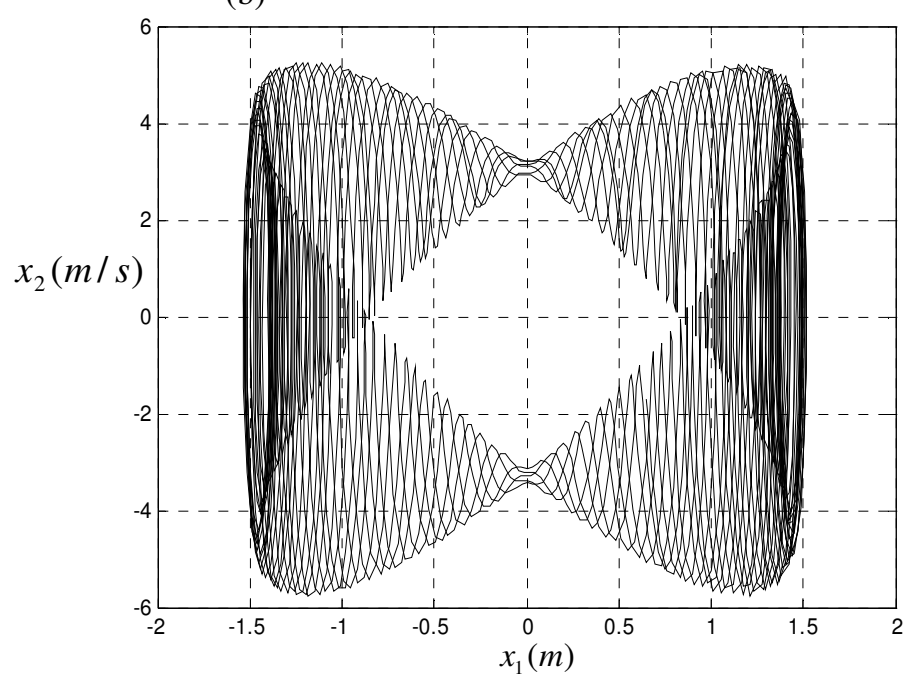

(c)

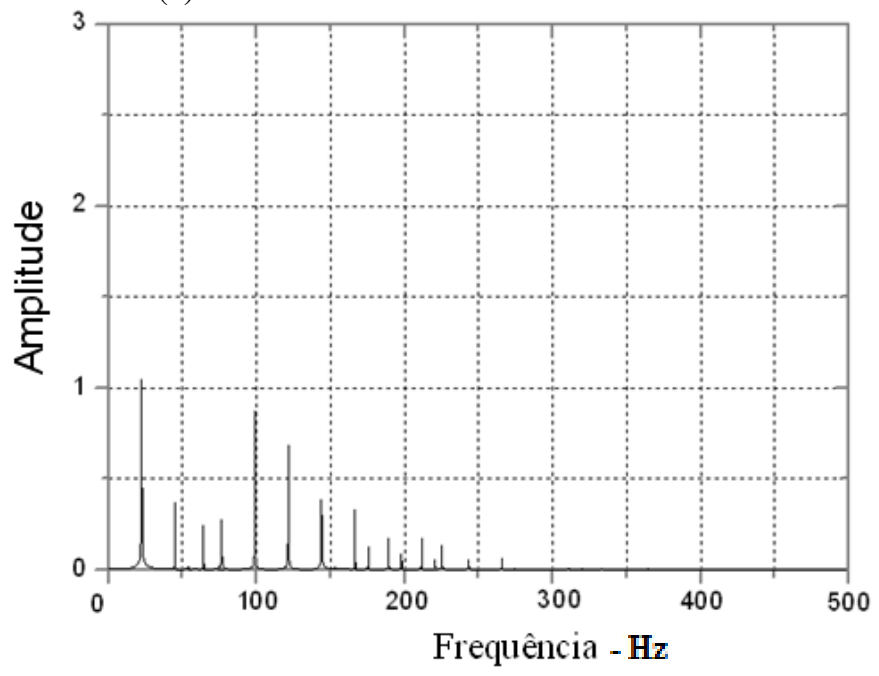

Figura 4.7 - (a) Histórico no tempo. (b) Plano de fase. (c) FFT.

Na Figura 4.7 nada pode ser afirmado sobre a estabilidade do sistema, pois de acordo com a análise da Figura 4.7(a) o histórico no tempo se mostra muito oscilante e muito irregular. Observa-se o mesmo efeito na Figura 4.7(b), onde o ciclo limite apresenta muitas oscilações, sendo assim, não se pode afirmar que exista um movimento caótico, pois o sistema também pode apresentar múltiplos períodos. Esta possível periodicidade deixa de existir quando se observa a FFT da Figura 4.7(c), pois os picos não apresentam essa característica.

$\mathrm{Na}$ análise seguinte, os expoentes de Lyapunov são calculados para analisar a estabilidade do sistema. 
A evolução dos expoentes de Lyapunov é apresentada na Figura 4.8 como segue.

$\Lambda_{1}$
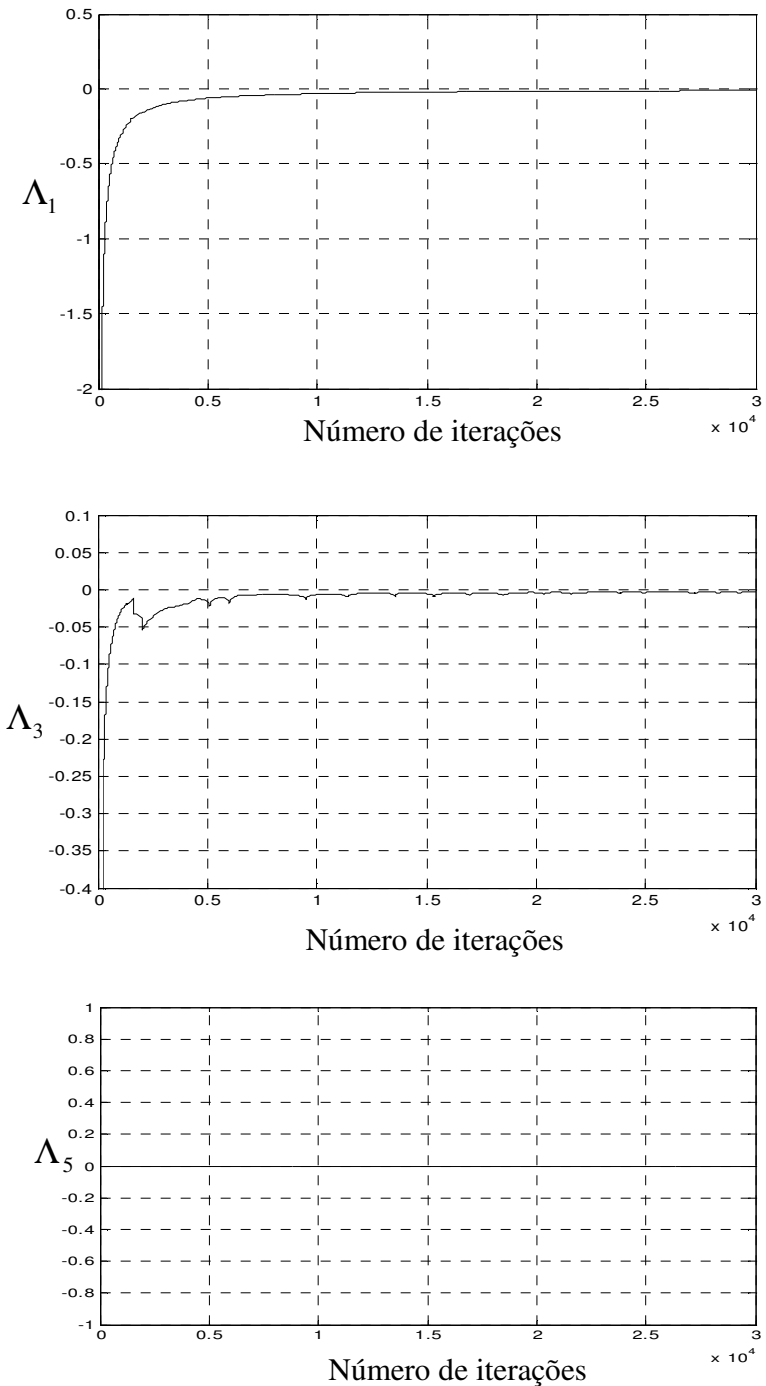

$\Lambda_{2}$
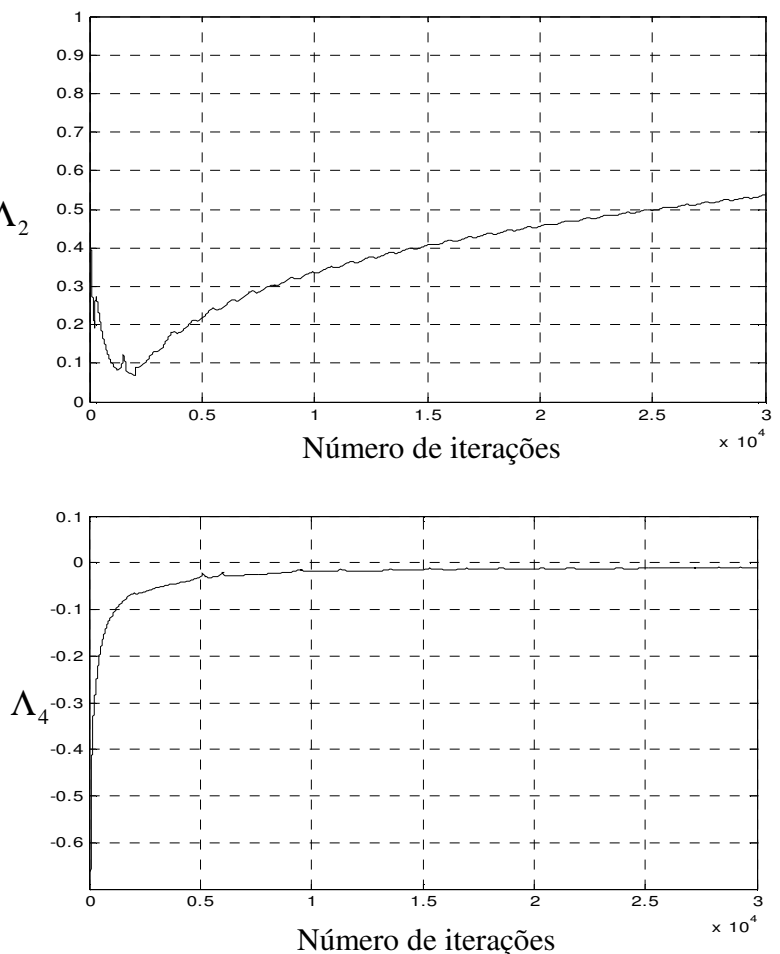

Figura 4.8 - Evolução dos expoentes de Lyapunov.

Para $T=4.5$, observa-se na Figura 4.8 que os valores dos expoentes de Lyapunov são:

Tabela 4.5 - Expoentes de Lyapunov para o torque do motor $T=4.5$.

\begin{tabular}{|l|l|}
\hline $\begin{array}{c}\text { Expoentes de } \\
\text { Lyapunov }(\Lambda)\end{array}$ & Valores \\
\hline$\Lambda_{1}$ & -0.0103 \\
\hline$\Lambda_{2}$ & +0.5360 \\
\hline$\Lambda_{3}$ & -0.0305 \\
\hline$\Lambda_{4}$ & -0.0108 \\
\hline$\Lambda_{5}$ & 0 \\
\hline
\end{tabular}


Desta forma, pode-se afirmar que o sistema deixou a condição de regularidade no movimento e começou a apresentar um comportamento caótico, pois um dos expoentes de Lyapunov mostrou-se positivo.

Analisa-se o comportamento do sistema quando o valor do torque aumenta para $T=6.5$, como segue.

O histórico no tempo, plano de fase e FFT são mostrados na Figura 4.9 como segue.

(a)

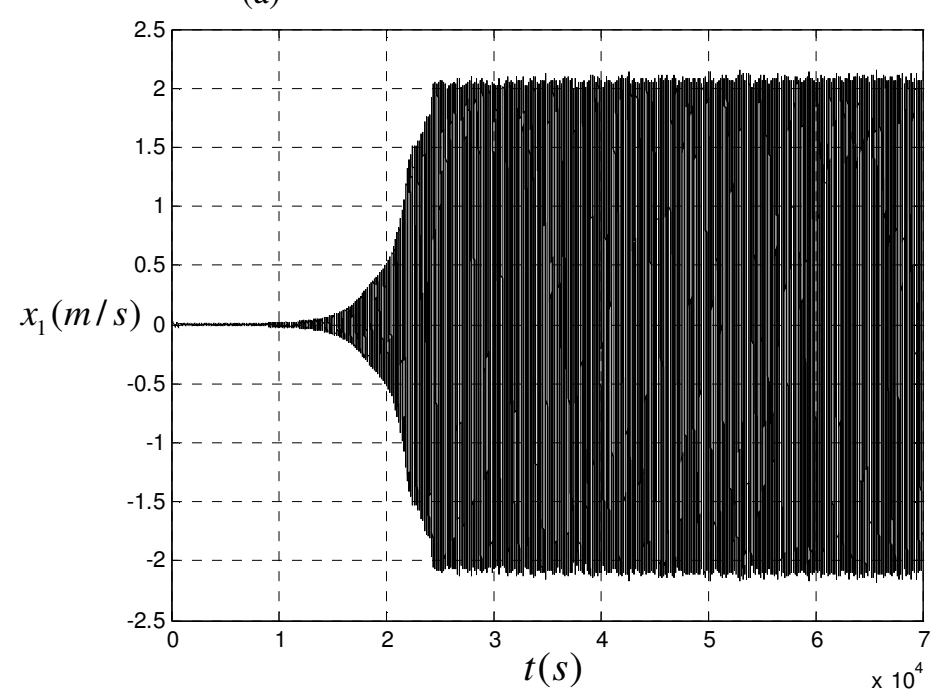

(b)

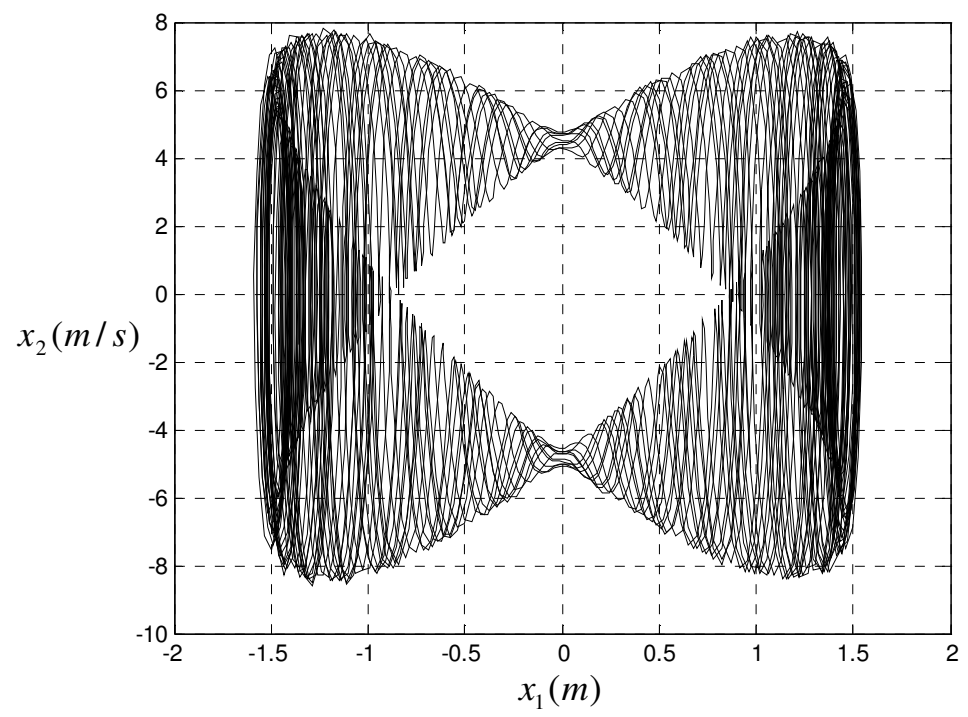


(c)

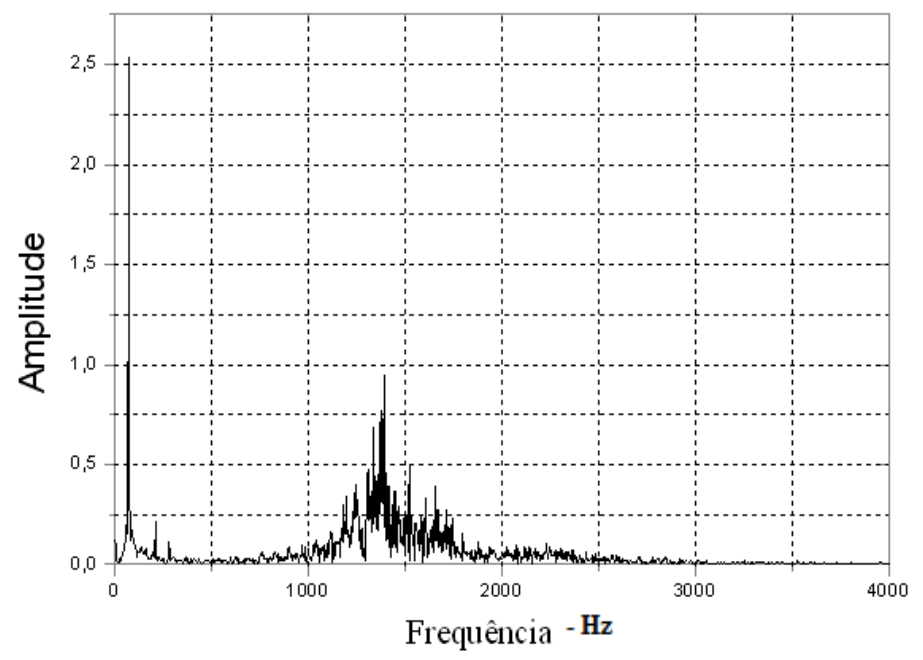

Figura 4.9 - (a) Histórico no tempo. (b) Plano de fase. (c) FFT.

Na Figura 4.9 podemos afirmar que o comportamento caótico permanece no sistema. A Figura 4.9(a) apresenta um histórico no tempo com amplitudes e oscilações mais elevadas. Observa-se que o ciclo limite da Figura 4.9(b) acompanha esse aumento nas oscilações do sistema. A FFT da Figura 4.9(c) apresenta diversos picos, não sendo possível observar nenhuma característica de periodicidade do sistema.

A evolução dos expoentes de Lyapunov é apresentada na Figura 4.10 como segue.
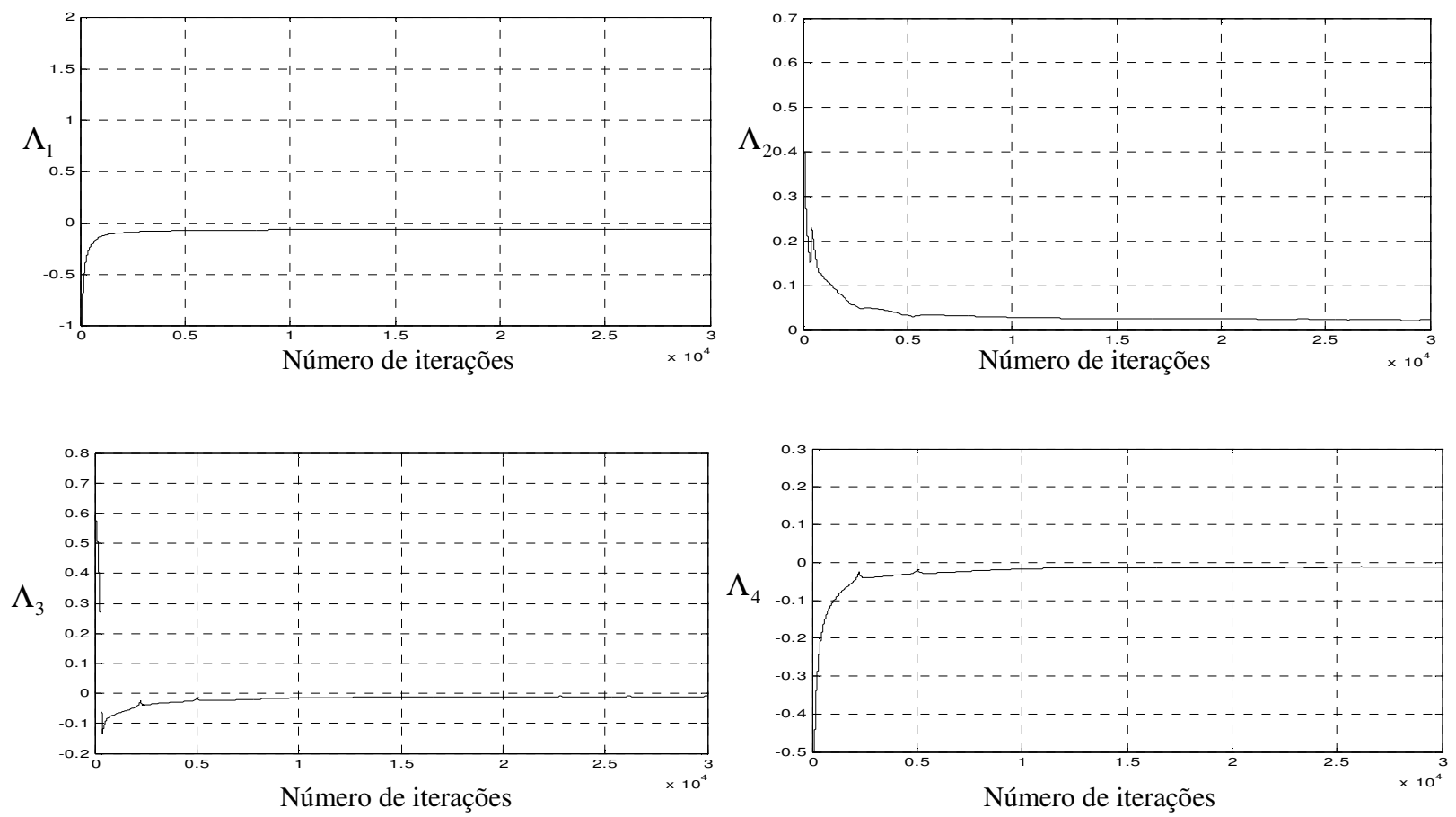


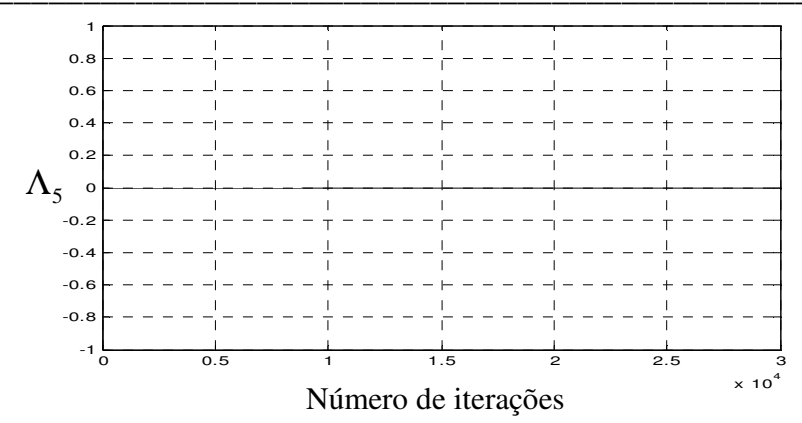

Figura 4.10 - Evolução dos expoentes de Lyapunov.

Para $T=6.5$ os valores dos expoentes de Lyapunov são:

Tabela 4.6 - Expoentes de Lyapunov para o torque do motor $T=6.5$.

\begin{tabular}{|l|l|}
\hline $\begin{array}{c}\text { Expoentes de } \\
\text { Lyapunov }(\Lambda)\end{array}$ & Valores \\
\hline$\Lambda_{1}$ & -0.0638 \\
\hline$\Lambda_{2}$ & +0.0227 \\
\hline$\Lambda_{3}$ & -0.0098 \\
\hline$\Lambda_{4}$ & -0.0119 \\
\hline$\Lambda_{5}$ & 0 \\
\hline
\end{tabular}

De acordo com os valores dos expoentes apresentados na Tabela 5, pode-se concluir que o sistema apresenta um comportamento caótico, pois pelo menos um expoente é positivo.

A tabela abaixo ilustra a evolução dos expoentes de Lyapunov em relação a cada variação do torque do motor mostrando apenas os sinais de cada expoente.

Tabela 4.7 - Sinais dos expoentes de Lyapunov para cada valor do torque do motor.

\begin{tabular}{|l|c|c|c|c|}
\hline \multirow{2}{*}{ Torque $(T)$} & \multicolumn{4}{|c|}{ Sinal dos expoentes de Lyapunov $(\Lambda)$} \\
\cline { 2 - 5 } & $\Lambda_{1}$ & $\Lambda_{2}$ & $\Lambda_{3}$ & $\Lambda_{4}$ \\
\hline 0.5 & - & - & - & - \\
\hline 1.5 & - & - & - & - \\
\hline 2.5 & - & - & - & - \\
\hline 4.5 & - & + & - & - \\
\hline 6.5 & - & + & - & - \\
\hline
\end{tabular}


A análise do sistema para verificar a presença do efeito Sommerfeld é apresentada no tópico que segue.

\subsection{Efeito Sommerfeld - Caso Não Ideal}

Sendo o sistema analisado um sistema não ideal, busca-se observar que a resposta do sistema influencia a fonte de energia, que por sua vez, é responsável pelo movimento da estrutura. Sendo assim, podemos afirmar que existe uma interação entre a fonte de excitação e o movimento da estrutura, sendo assim o torque do motor $(T)$ não será mais um valor fixo, como mostrado na seção anterior, e sim uma função do primeiro grau, já que a relação entre a tensão do motor $\left(T_{E}\right)$ x número de rotação do motor sem o desbalanceamento é linear.

$$
T=T_{E}-x_{4} .
$$

O gráfico apresentado a seguir, mostra que o sistema apresenta o efeito Sommerfeld.

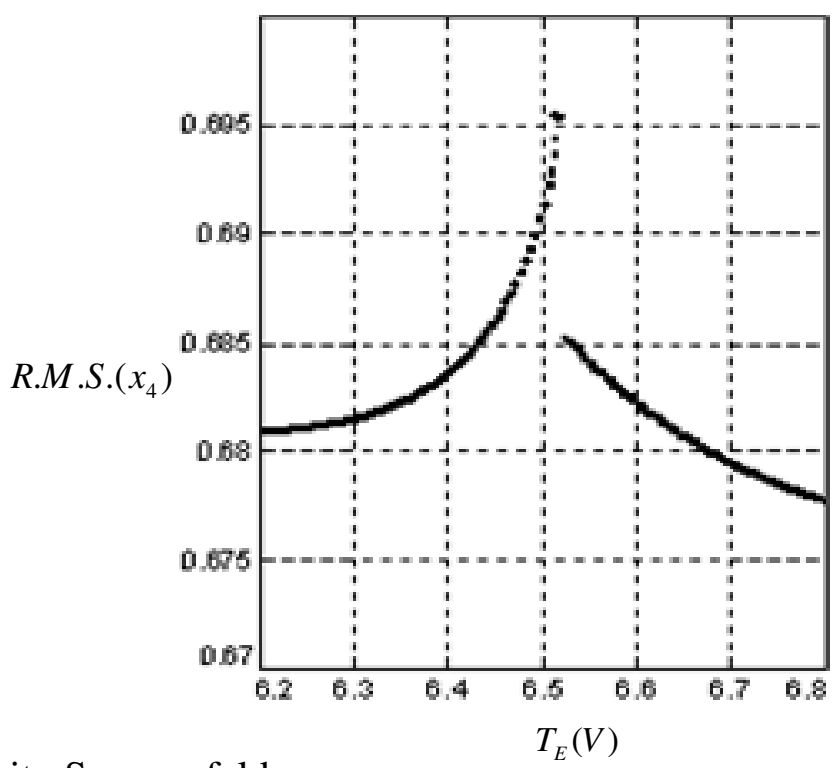

Figura 4.11 - Efeito Sommerfeld.

Analisando-se o gráfico apresentado na Figura 4.11, observa-se que à medida que a tensão elétrica aumenta, a amplitude cresce consideravelmente. Observa-se ainda, que um pequeno acréscimo na tensão elétrica do motor $(T=6.51 \mathrm{~V})$ causa uma variação brusca no movimento, ocasionando uma significativa diminuição na amplitude de vibração do sistema. Sendo que essa diminuição brusca de amplitude deve-se exclusivamente ao desbalanceamento da massa que foi acoplado ao motor. Desta forma, conclui-se que o sistema apresentou o efeito Sommerfeld. 


\section{Capítulo 5}

\section{Ensaio Experimental}

Este capítulo apresenta todo procedimento experimental referente ao modelo do sistema apresentado no Capítulo 3, que consiste numa viga engastada com um motor desbalanceado na extremidade oposta ao engaste. O objetivo do experimento é analisar o comportamento dinâmico do sistema ao se alterar o parâmetro de controle, que neste caso, corresponde a tensão $T_{E}$ do motor.

Os ensaios experimentais foram divididos em duas análises: caso ideal e caso não ideal. Para o caso ideal considerou-se uma tensão fixa para cada aquisição dos dados e os gráficos apresentados e analisados são: Histórico no tempo, plano de fase reconstruído (Simoni, 2008), expoente de Lyapunov (Simoni, 2008) e FFT. Para o caso não ideal, pretende-se observar o efeito Sommerfeld e obter os gráficos que ilustram o efeito de variação da amplitude de vibração da viga em relação a variação da tensão do motor. A tensão fornecida ao motor foi escolhida como variável que controla a ocorrência de diferentes modos de oscilação do sistema.

No Apêndice E é apresentado um exemplo clássico encontrado na literatura do procedimento para a reconstrução do espaço de fase do atrator de Rössler (Alligood, 1996) e (Ferrara, 1995).

\subsection{Aparato Experimental}

Para a realização do experimento, foi utilizada uma viga de aço, cujas dimensões são: $400 \mathrm{~mm}$ de comprimento, $35 \mathrm{~mm}$ de largura e $1 \mathrm{~mm}$ de espessura. $\mathrm{O}$ motor utilizado nos ensaios foi ligado a uma fonte de potência, o que permite a variação da tensão do motor. $\mathrm{O}$ modelo do motor, assim como os seus parâmetros físicos, encontram-se detalhados no Apêndice D.

Para a aquisição dos dados nos ensaios experimentais, foi utilizado o sistema de aquisição e análise de dados Tektronics.2630 Fourier Analyzer com frequência de aquisição de $2 \mathrm{kHz}, 4096$ pontos em cada janela de tempo; além de dados no domínio do tempo, o autoespectro da aceleração foi calculado usando-se janela hanning e 10 médias lineares com trigger manual. Para realizar medidas de vibração do sistema, foi utilizado um acelerômetro 
PCB piezotronics modelo 333B $\mathrm{ICP}^{\circledR}$ de sensibilidade $100 \mathrm{mV} / \mathrm{g}$. Os parâmetros físicos do experimento são apresentados abaixo.

Tabela 5.1 - Parâmetros físicos do experimento.

\begin{tabular}{|l|l|}
\hline $\begin{array}{l}\text { Massa (Viga+motor } \\
\text { desbalanceado) }\end{array}$ & $M=0.5 \mathrm{~kg}$ \\
\hline Densidade linear da viga & $\rho \cdot A=0.4 \mathrm{~kg} / \mathrm{m}$ \\
\hline Rigidez & $E \cdot I=1.01 \mathrm{Nm}^{2}$ \\
\hline Comprimento da viga & $L=0.4 \mathrm{~m}$ \\
\hline Raio do disco desbalanceado & $r=4 \cdot 10^{-2} \mathrm{~m}$ \\
\hline
\end{tabular}

O esquema da montagem experimental é apresentado como segue.

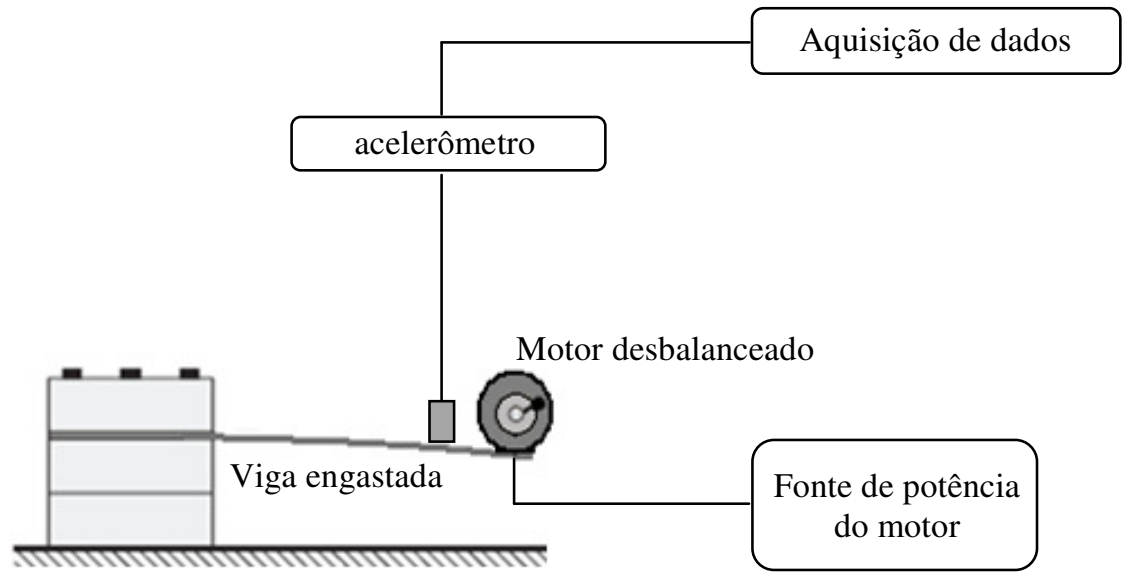

Figura 5.1 - Esquema da montagem experimental do sistema descrito no Capítulo 3.

As fotos do aparato experimental são mostradas como segue.

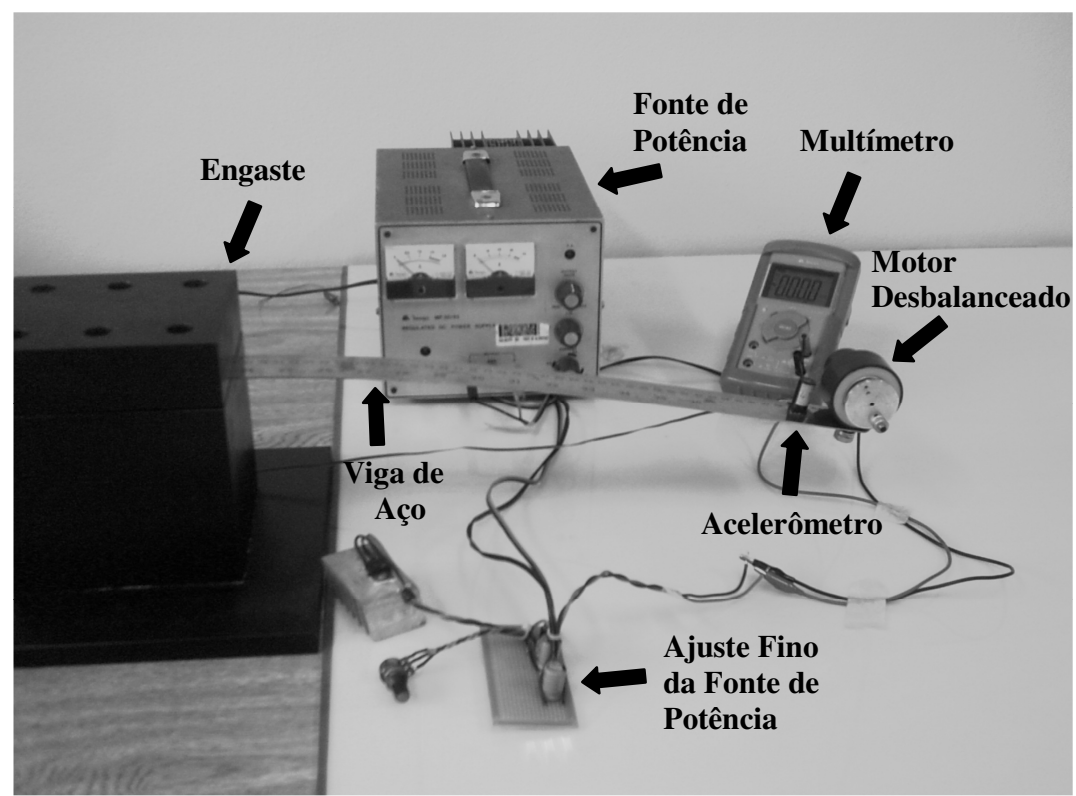




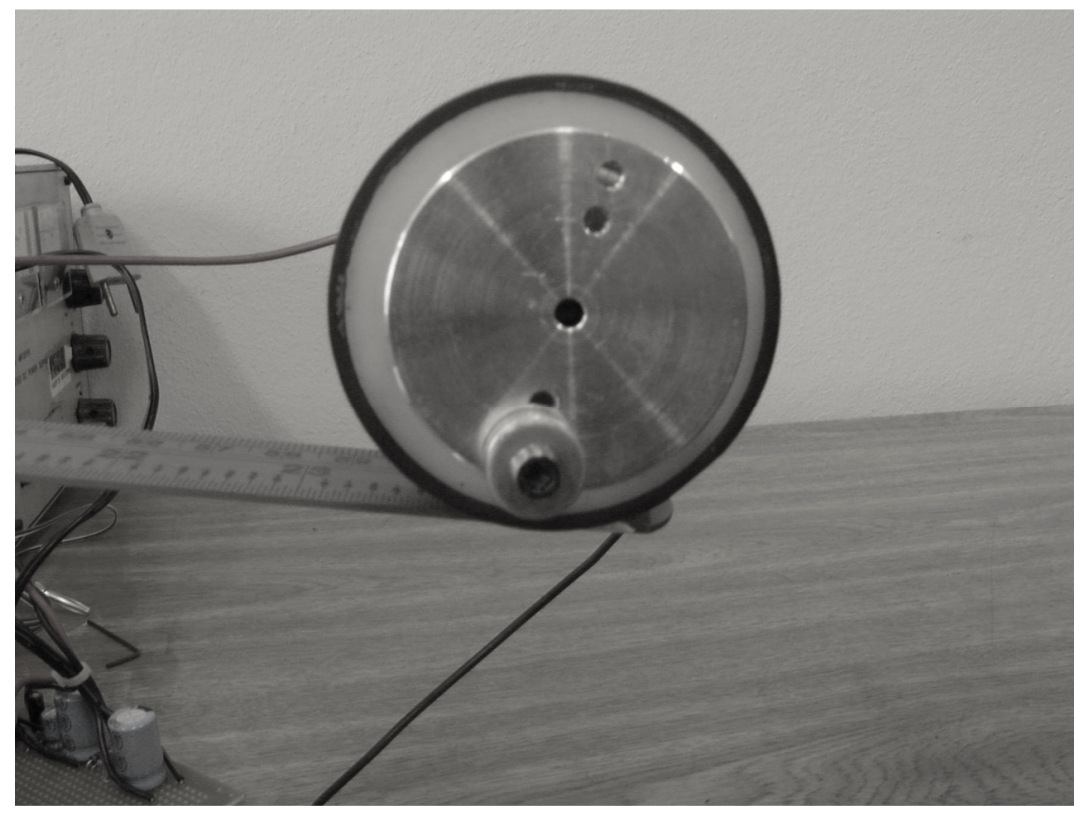

Figura 5.2 - Fotos do experimento.

Na seção que segue, são apresentados os resultados experimentais, juntamente com a discussão dos resultados.

\subsection{Resultados Experimentais}

\subsubsection{Caso Ideal}

Os resultados experimentais apresentados descrevem a dinâmica do sistema considerado como sendo uma aproximação de um sistema ideal, ou seja, escolhe-se uma tensão fixa para o motor e, em seguida, liga-se o sistema até que o mesmo entre em regime permanente, por fim inicia-se a aquisição dos dados experimentais. As tensões utilizadas nas análises foram: $T_{E}=0.5 \mathrm{~V}, 1.5 \mathrm{~V}, 4.5 \mathrm{~V}$ e $6.5 \mathrm{~V}$. 
O histórico no tempo, plano de fase reconstruído e FFT para $T_{E}=0.5 \mathrm{~V}$ são apresentados na Fig. 5.3.

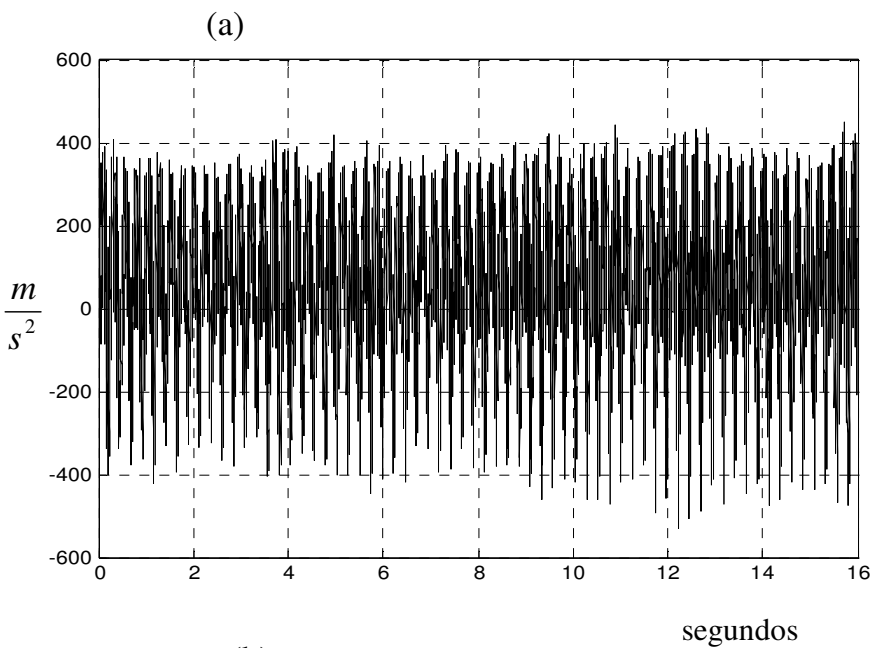

(b)

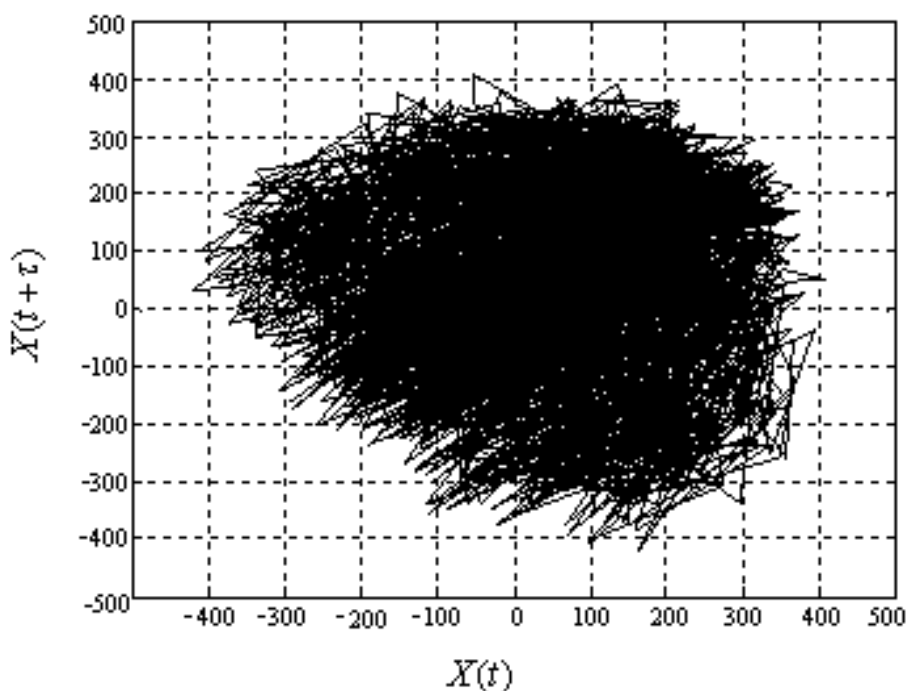

(c)

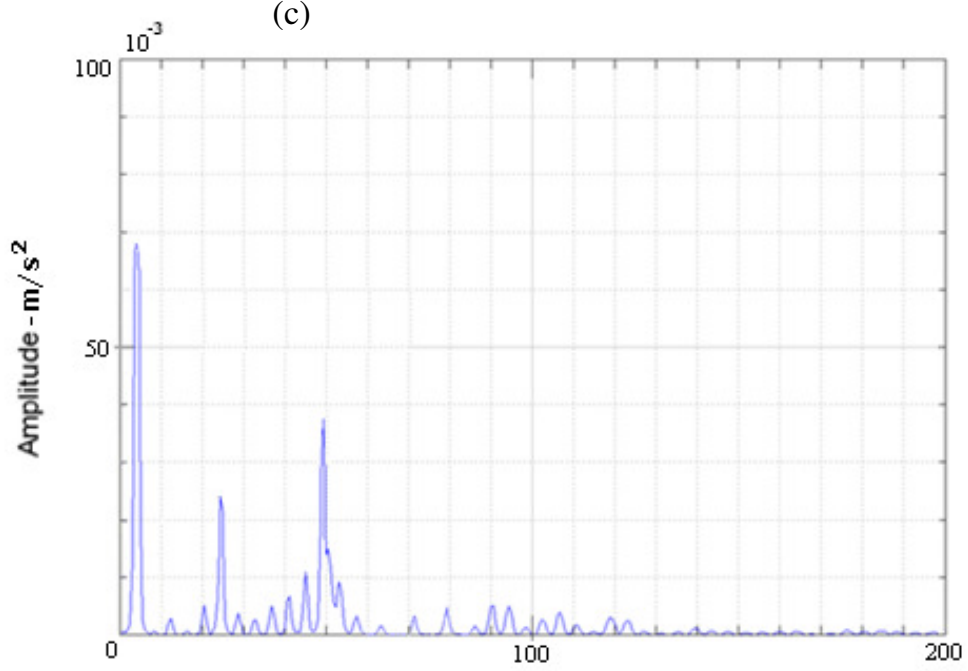

Frequência - $\mathbf{H z}$

Figura $5.3-T_{E}=0.5 \mathrm{~V}$. (a) Histórico no tempo. (b) Plano de fase reconstruído, sendo $t$ a série temporal e $\tau=123$ a defasagem. (c) FFT. 
A Fig. 5.3(a) mostra um histórico no tempo com oscilações regulares, o que indica que o sistema está em regime permanente. Na Fig. 5.3(b) observa-se que o plano de fase reconstruído descreve uma órbita fechada que corresponde um ciclo limite, observa-se também a presença de ruído no sinal obtido, não comprometendo de forma significativa a qualidade do resultado. Na Fig. 5.3(c) a FFT apresenta três pequenos picos em destaque, o que pode caracterizar que o sistema é periódico e de período três.

A análise que caracteriza o comportamento caótico do sistema, para este valor de tensão, é feita através do cálculo do maior expoente de Lyapunov como segue.

Tabela 5.2 - Maior valor do expoente de Lyapunov para a tensão do motor $T_{E}=0.5 \mathrm{~V}$.

\begin{tabular}{|c|c|}
\hline Maior Expoente de Lyapunov $(\Lambda)$ & Valor \\
\hline$\Lambda_{1}$ & -0.4242 \\
\hline
\end{tabular}

Analisando a Tabela 5.2 pode-se afirmar que, para a tensão $T_{E}=0.5 \mathrm{~V}$, o sistema apresenta um comportamento regular, pois o expoente de Lyapunov tem sinal negativo.

$\mathrm{Na}$ análise seguinte, apresenta-se o comportamento do sistema quando o valor da tensão aumenta para $T_{E}=1.5 \mathrm{~V}$, como mostrado na Fig. 5.4.

O gráfico do histórico no tempo, plano de fase reconstruído e FFT são mostrados como segue.

(a)

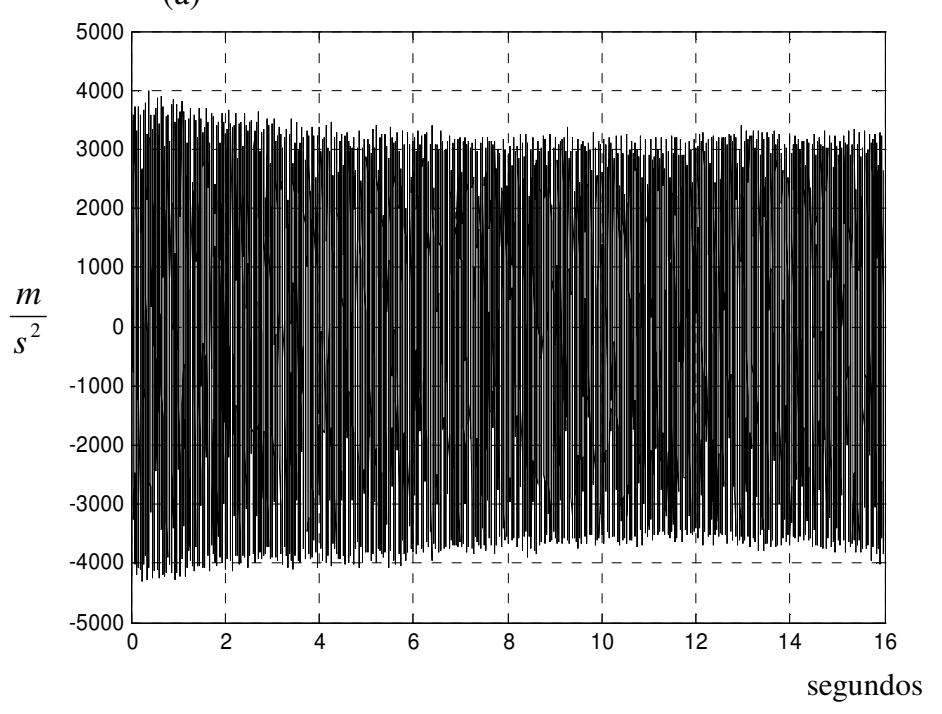


(b)

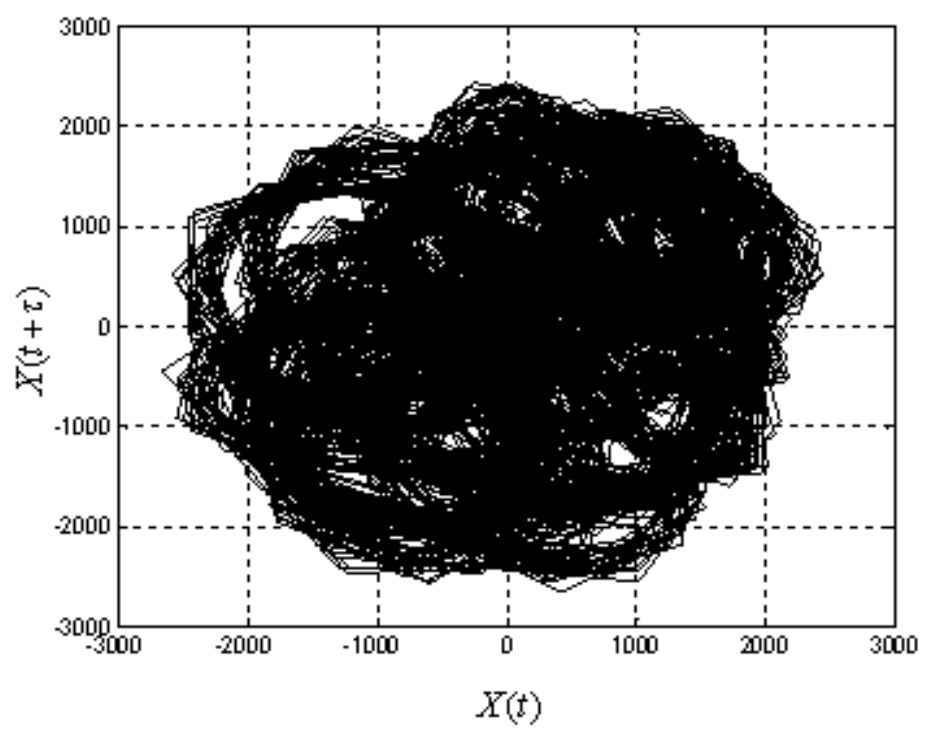

(c)

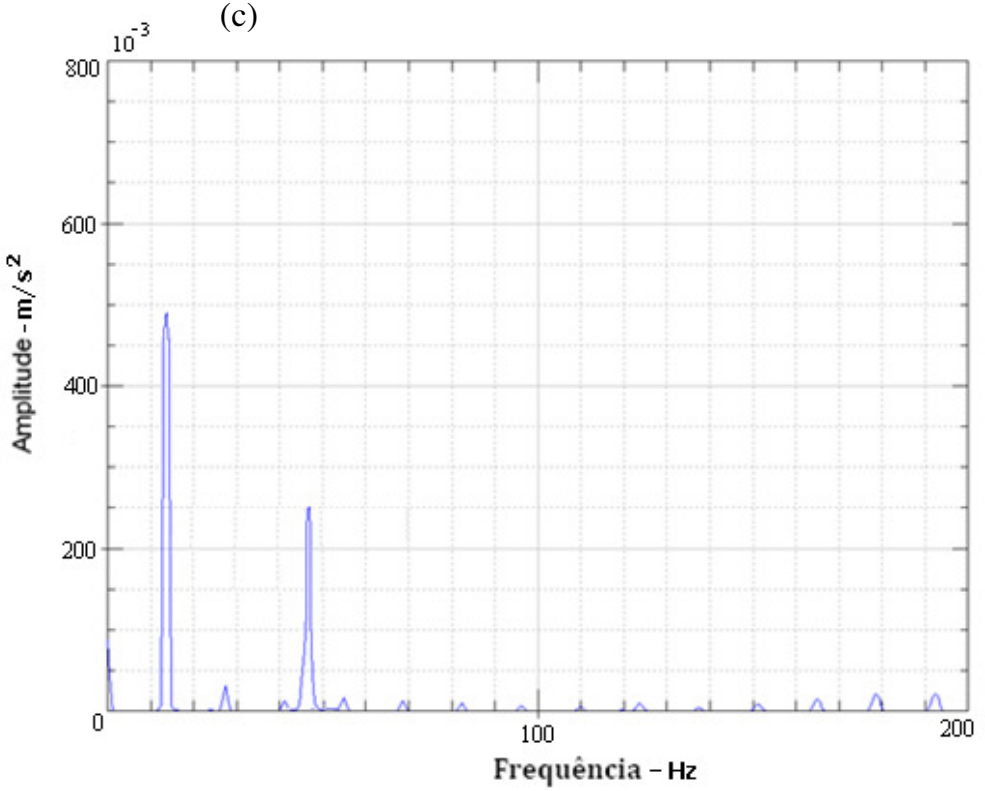

Figura $5.4-T_{E}=1.5 \mathrm{~V}$. (a) Histórico no tempo. (b) Plano de fase reconstruído, sendo $t$ a série temporal e $\tau=32$ a defasagem. (c) FFT.

Na Fig. 5.4 (a) observa-se que o histórico no tempo apresentou um aumento de amplitude. Na Fig. 5.4(b) observa-se um aumento de amplitude no plano de fase reconstruído devido ao aumento na tensão do motor e o plano de fase apresenta um ciclo limite. Na Fig. 5.4(c) a FFT mostra dois picos bem definidos, o que pode caracterizar que o sistema é periódico e de períodos dois.

Para verificar a presença de caos no sistema calcula-se o maior expoente de Lyapunov como mostrado na Tabela 5.3 
Tabela 5.3 - Maior valor do expoente de Lyapunov para a tensão do motor $T_{E}=1.5 \mathrm{~V}$.

\begin{tabular}{|c|c|}
\hline Maior Expoente de Lyapunov $(\Lambda)$ & Valor \\
\hline$\Lambda_{2}$ & -0.2140 \\
\hline
\end{tabular}

Desta forma, pode-se afirmar que o sistema apresenta um comportamento regular, pois o maior expoente de Lyapunov é negativo.

$\mathrm{Na}$ análise que segue, o valor da tensão é aumentado para $T_{E}=4.5 \mathrm{~V}$.

O histórico no tempo, plano de fase e FFT são apresentados na Fig. 5.5 como segue.

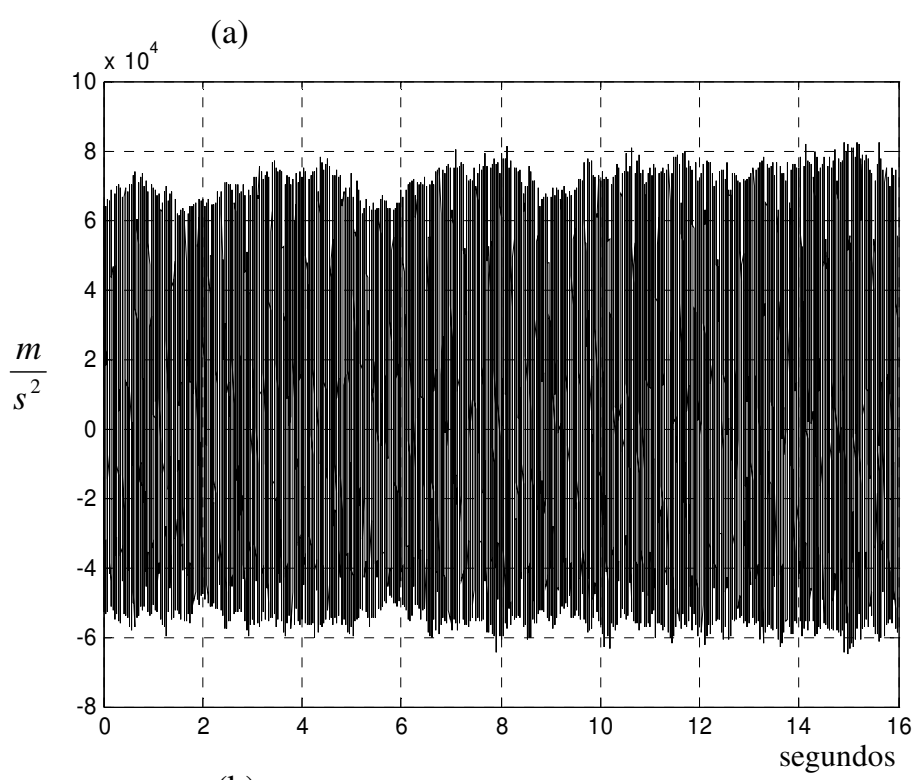

(b)

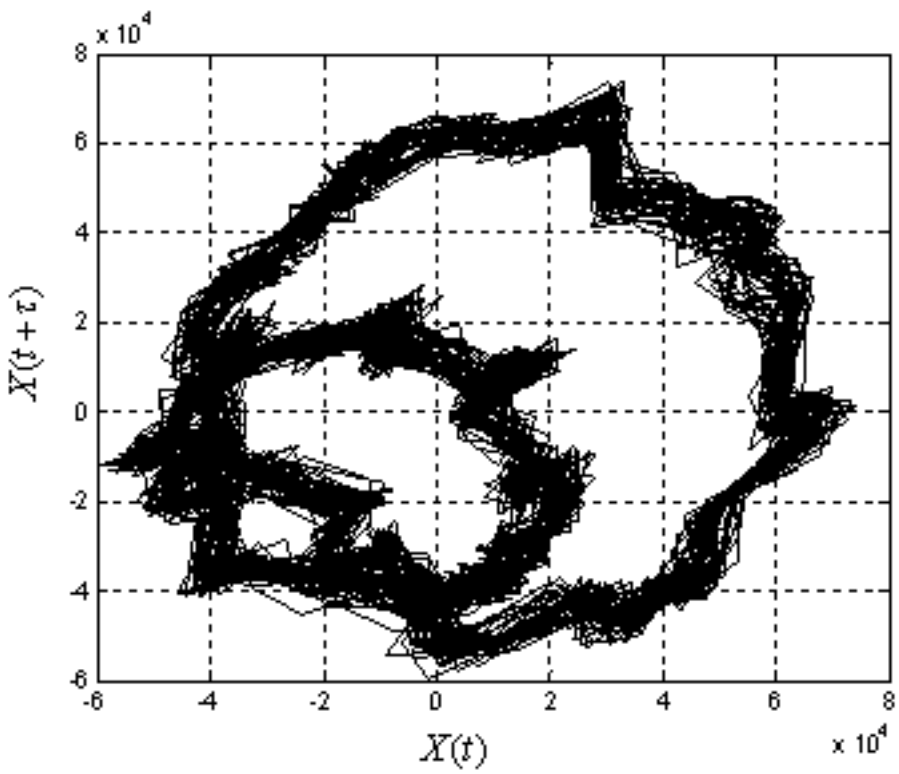


(c)

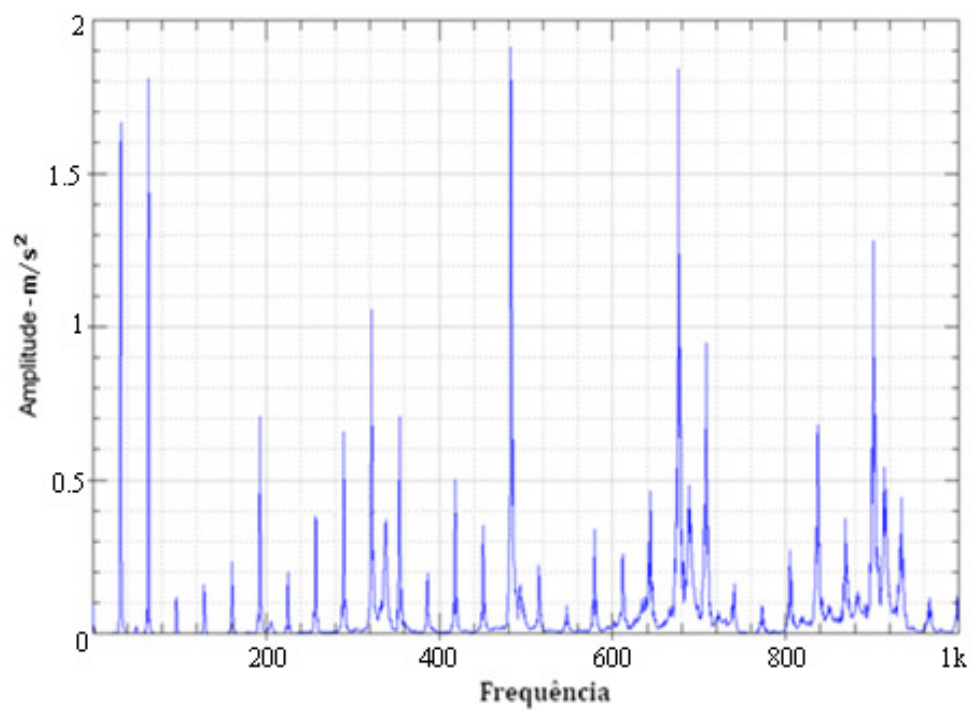

Figura $5.5-T_{E}=4.5 \mathrm{~V}$. (a) Histórico no tempo. (b) Plano de fase reconstruído, sendo $t$ a série temporal e $\tau=15$ a defasagem. (c) FFT.

Na Fig. 5.5 nada pode ser afirmado sobre a estabilidade do sistema, pois de acordo com a análise da Fig. 5.5(a) o histórico no tempo mostra oscilações irregulares. Na Fig. 5.5(b), observa-se mais um aumento na amplitude e nas oscilações no ciclo limite do plano de fase reconstruído. A possível periodicidade do sistema deixa de existir quando se observa a FFT da Fig. 5.5(c), pois os picos não apresentam nenhuma característica de periodicidade, estando a energia distribuída em uma banda mais larga de frequência.

$\mathrm{Na}$ análise seguinte, o maior expoente de Lyapunov é calculado para verificar se o sistema apresenta um comportamento caótico, como mostrado na Tabela 5.4.

Tabela 5.4 - Maior valor do expoente de Lyapunov para a tensão do motor $T_{E}=4.5 \mathrm{~V}$.

\begin{tabular}{|c|c|}
\hline Maior Expoente de Lyapunov $(\Lambda)$ & Valor \\
\hline$\Lambda_{3}$ & +0.272 \\
\hline
\end{tabular}

Desta forma, pode-se afirmar que o sistema deixou a condição de regularidade no movimento e começou a apresentar um comportamento caótico, pois o expoente de Lyapunov se mostrou positivo.

Analisa-se o comportamento do sistema quando o valor da tensão do motor aumenta para $T_{E}=6.5 \mathrm{~V}$, como segue. 
O histórico no tempo, plano de fase e FFT são mostrados na Fig. 5.6 como segue.

(a)
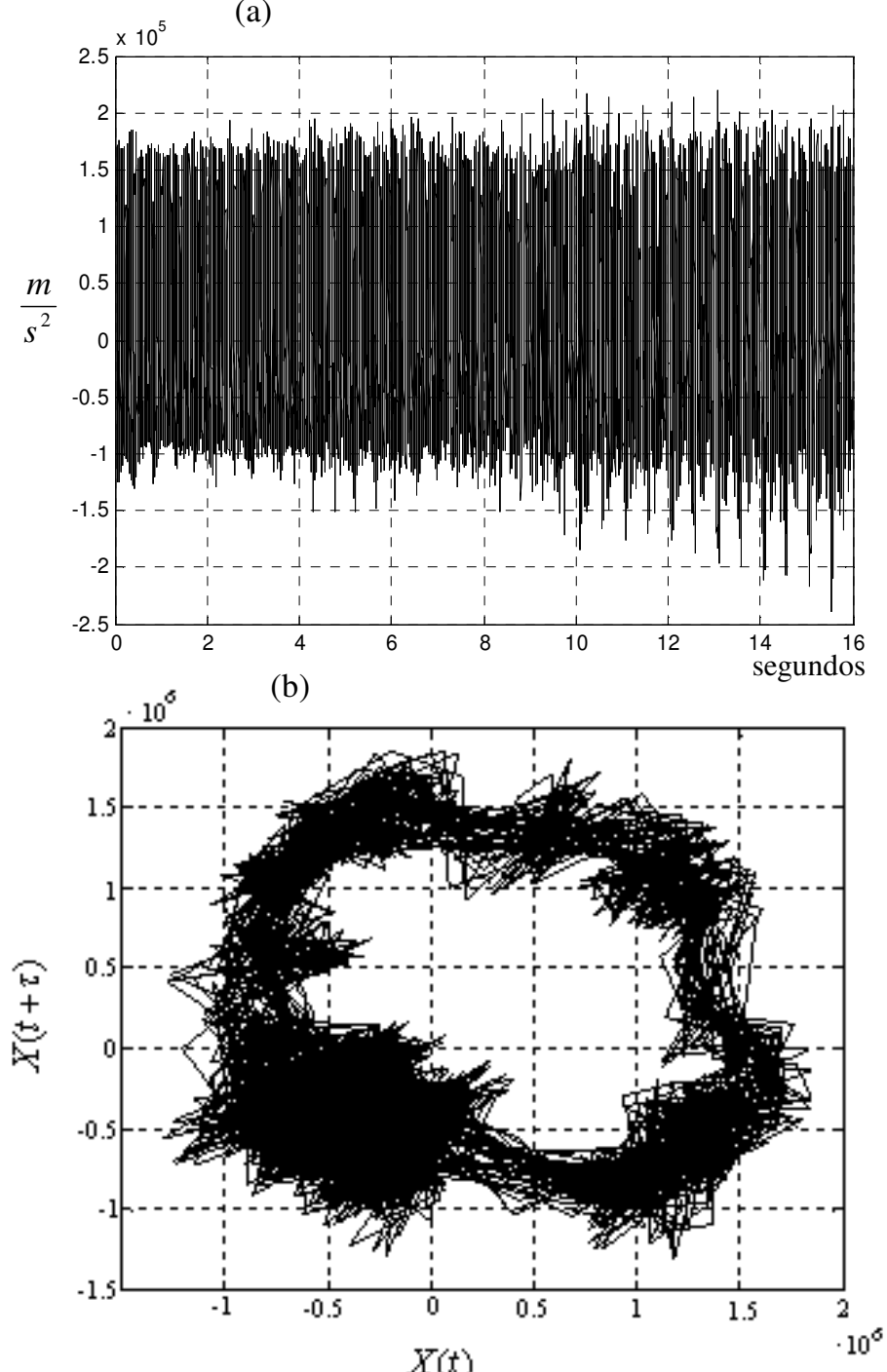

(c)

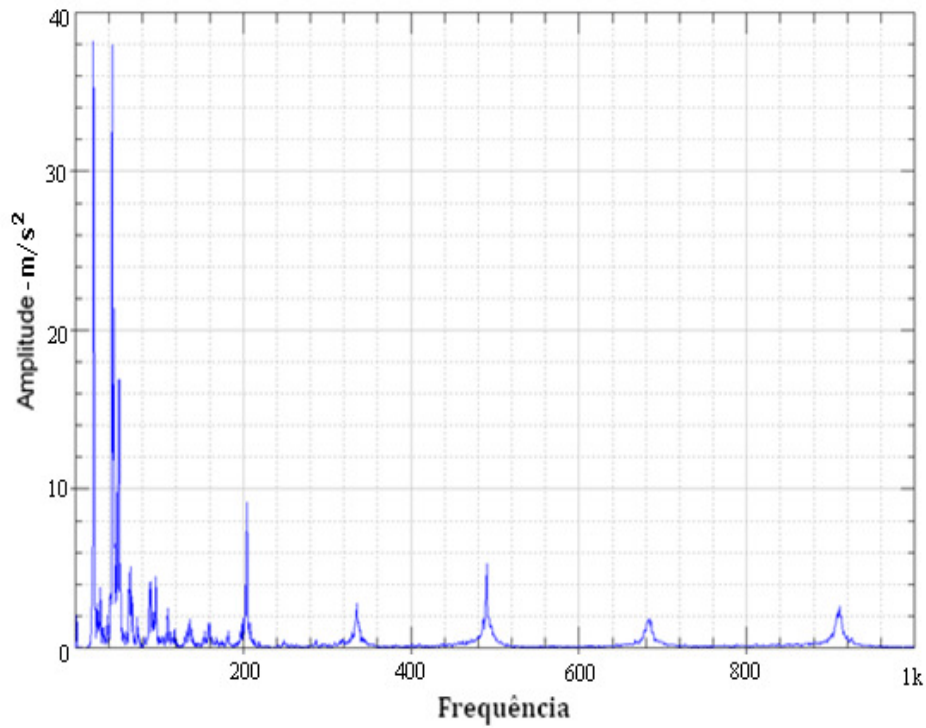

Figura 5.6 - $T_{E}=6.5 \mathrm{~V}$. (a) Histórico no tempo. (b) Plano de fase reconstruído, sendo $t$ a série temporal e $\tau=19$ a defasagem. (c) FFT. 
A Fig. 5.6(a) apresenta um histórico no tempo com amplitudes e oscilações irregulares mais elevadas. Observa-se uma mudança e novamente um aumento no ciclo limite da Fig. 5.6(b). Essa mudança na forma do atrator sugere que o sistema passa por bifurcações (Simoni 2008). A FFT da Fig. 5.6(c) apresenta diversos picos, não sendo possível observar nenhuma característica de periodicidade do sistema. Novamente, observa-se que a energia está distribuída em bandas mais larga de frequência.

O cálculo do maior expoente de Lyapunov é apresentado na Tabela 5.5 como segue.

Tabela 5.5 - Maior valor do expoente de Lyapunov para a tensão do motor $T_{E}=6.5 \mathrm{~V}$.

\begin{tabular}{|c|c|}
\hline Maior Expoente de Lyapunov $(\Lambda)$ & Valor \\
\hline$\Lambda_{4}$ & +0.338 \\
\hline
\end{tabular}

De acordo com os valores dos expoentes apresentados na Tabela 5.5, pode-se concluir que o sistema apresenta um comportamento caótico, o expoente de Lyapunov é positivo.

A análise do sistema para verificar a presença do efeito Sommerfeld é apresentada no tópico que segue.

\subsubsection{Caso Não Ideal - Salto Experimental}

Usando o mesmo aparato experimental, na tentativa de observar o efeito Sommerfeld, variou-se a tensão do motor desbalanceado.

Para observar o fenômeno do salto, variou-se lentamente (aumentando e depois diminuindo) a tensão do motor, por meio da fonte de tensão em que o mesmo se encontra ligado.

Para a aquisição dos dados do experimento e obtenção da curva que ilustra o efeito Sommerfeld, escolheu-se uma faixa de tensão do motor de $T_{E}=0.5 \rightarrow 14 \mathrm{~V}$ com um passo de $1 V$. A cada variação do passo escolhido para a tensão, um vetor de dados foi obtido através da utilização do acelerômetro conectado à viga, que compõe o sistema representado na Fig. 5.1. Este conjunto de vetores registra a amplitude das oscilações, que ocorrem devido à variação na tensão do motor desbalanceado.

Com o objetivo de observar o efeito Sommerfeld, utilizaram-se os vetores de dados obtidos a partir do experimento acima descrito. 
A Fig. 5.7 ilustra a amplitude de vibração em função da variação da tensão do motor desbalanceado, como segue.

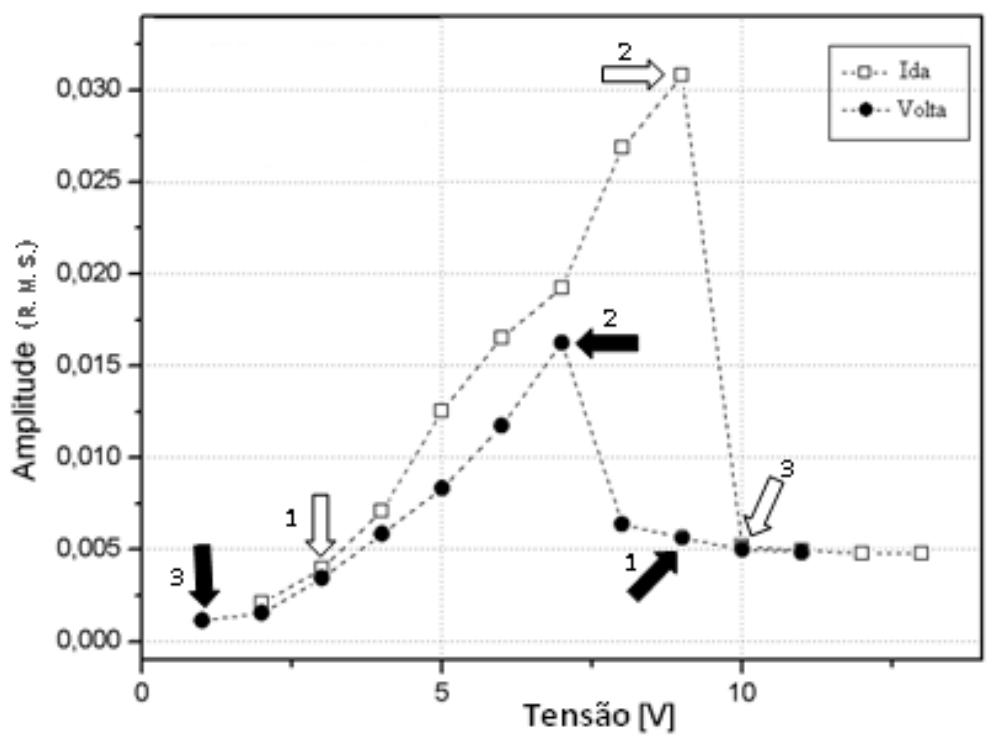

Figura 5.7 - Efeito Sommerfeld para o aumento (Ida) e redução (Volta) da Tensão do motor desbalanceado.

Observa-se na Fig. 5.7 que com o aumento da tensão no motor (Ida) a amplitude do sistema aumenta. Isto ocorre por consequiência do desbalanceamento do motor. No entanto, a amplitude diminui bruscamente numa faixa de tensão entre 9 a 10V. Este fenômeno evidencia o aparecimento do efeito Sommerfeld, ou seja, ocorreu um salto no sistema, fazendo com que o valor da amplitude de vibração da viga diminua de forma muita rápida. Observa-se também que com a diminuição da tensão no motor desbalanceado (Volta) o sistema torna a saltar numa faixa entre 6 a $8 \mathrm{~V}$, acompanhado do aumento na amplitude de vibração da viga.

A análise que segue está relacionada com os pontos destacados na Fig. 5.7, indicados através das "setas vazias" e "setas cheias", onde se apresenta a evolução da amplitude de vibração do sistema em relação ao tempo antes e depois do salto ocorrido no sistema. A Figura 5.8 ilustra esta análise. 
(a) Ida

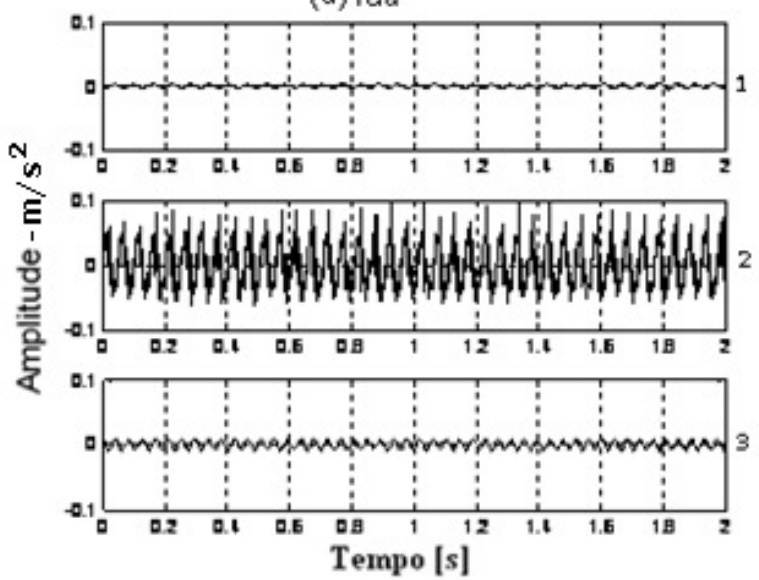

(b) Volta
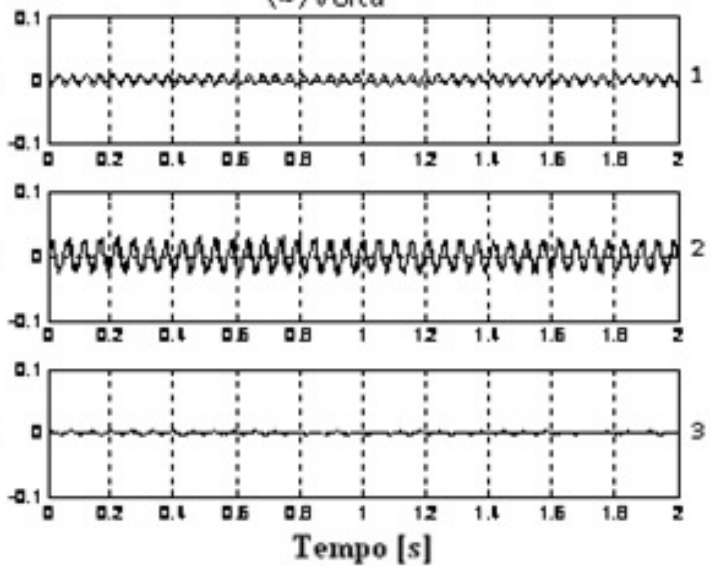

Figura 5.8 - (a) Variação da amplitude em função do tempo para o aumento da voltagem (Ida). (b) Amplitude versus Tempo para a redução da voltagem (Volta).

Observa-se na Fig. 5.8(a) que o gráfico 1 apresenta uma baixa amplitude de vibração, isso pode ser comprovado ao observar a "seta vazia" representada pelo $\mathrm{n}^{0} 1$ na Fig. 5.7. $\mathrm{O}$ gráfico 2 da Fig. 5.8(a) não apresenta o mesmo efeito, pois sua amplitude aumentou devido ao aumento de tensão no motor. Observa-se esse comportamento na Fig. 5.7, onde a "seta vazia" está representada pelo $\mathrm{n}^{0} 2$. Uma brusca diminuição da amplitude de vibração do sistema é observada no gráfico 3 da Fig. 5.8(a), sendo esse comportamento observado na "seta vazia" da Fig. 5.7 representada pelo $n^{0}$ 3. Desta forma, conclui-se que o sistema apresentou o efeito Sommerfeld. A mesma análise pode ser feita ao observar a Fig. 5.8(b) e relacionar as amplitudes de vibração com as "setas cheias" da Fig. 5.7.

No capítulo que segue apresentam-se as conclusões do trabalho e sugestões para propostas futuras. 


\section{Capítulo 6}

\section{Conclusões e Sugestões para Trabalhos Futuros}

\subsection{Conclusões}

Neste trabalho desenvolveu-se a modelagem matemática de uma viga engastada com um motor desbalanceado na extremidade oposta ao engaste. Nesta, considerou-se um modelo com o maior número de variáveis possíveis, de forma a evitar um excesso nas simplificações. Conseqüentemente, tanto a modelagem matemática quanto os resultados apresentados não ficaram comprometidos.

A modelagem matemática foi iniciada a partir das análises das forças atuantes no sistema, utilizando-se as leis de Newton para a obtenção das equações que descrevem o movimento do sistema. Optou-se por buscar soluções analíticas para as equações a partir de técnicas de perturbação e discretização, a fim de se ter um maior entendimento da solução do sistema.

O modelo matemático apresentado mostrou-se muito complexo devido à consideração de um número excessivo nas variáveis do problema. Por outro lado, por se tratar de um modelo consistente, contribuiu de forma significativa para o desenvolvimento e aprimoramento de sistemas vibracionais, uma vez que o conjunto de equações diferenciais obtido é geral, ou seja, permite expandir as equações de forma a escolher com quantos modos de vibrar se deseja estudar.

A validação do modelo desenvolvido foi feita, comparando-se o primeiro modo de vibrar do modelo não ideal e não amortecido de Duffing, pois os trabalhos encontrados na literatura corrente são baseados nesse modelo. A partir desta comparação, foi possível identificar o surgimento de termos adicionais no modelo desenvolvido, os quais não estão 
presentes no modelo de Duffing. Esses termos adicionais aparecem como consequiência da não simplificação das variáveis, que compõe o sistema.

Em relação aos resultados de simulação numérica, considerando-se primeiramente o parâmetro de controle, o torque, como sendo fixo (sistema ideal), os resultados mostraram a evolução dinâmica do sistema, ou seja, para quais valores do parâmetro de controle o sistema apresentava um comportamento regular até a obtenção de um comportamento caótico. Por outro lado, considerando-se o torque com sendo dependente da dinâmica do sistema (caso não ideal), o resultado mostrou a presença do efeito Sommerfeld no sistema. A obtenção dos resultados de simulação numérica exigiu um grande esforço computacional, de forma a tornar as análises muito lentas, em função do excessivo tempo de espera para o término dos gráficos.

Quanto à parte experimental deste trabalho, foi montado o protótipo de uma semiasa, com um motor desbalanceado acoplado na extremidade livre. Os resultados apresentados mostraram a evolução dinâmica do sistema, além da presença de caos no experimento, utilizando-se a técnica de reconstrução do espaço de fase e cálculo do expoente de Lyapunov. A presença do efeito Sommerfeld no sistema foi verificada na análise do sistema não ideal. Os resultados experimentais apresentaram resultados satisfatórios, quando comparados, de forma qualitativa, com os resultados obtidos nas simulações numéricas.

\subsection{Sugestões para Trabalhos Futuros}

Por este trabalho apresentar grande margem de aplicabilidade, e considerando-se sua complexidade e diversas ramificações, nesta seção são apresentadas algumas sugestões para pesquisas futuras, as quais se encontram enumeradas na sequência:

1- Verificar, através de análises de simulações numéricas e experimentais, qual a real importância dos termos adicionais que aparecem no modelo, quando comparado com o modelo de Duffing. 
2- Verificar o comportamento dinâmico do sistema, quando se expande a equação geral até o segundo e terceiro modos de vibrar;

3- Alterar a posição do motor na viga e analisar o comportamento dinâmico do sistema, tanto na parte teórica, quanto na parte experimental;

4- Estudar o modelo matemático, considerando-se um sistema com dois motores, a fim de obter novas verificações numéricas e experimentais;

5- Aplicar um sistema de controle no modelo, quando o sistema apresentar um comportamento caótico. 


\section{Capítulo 7}

\section{Referências Bibliográficas}

Alligood, K.T, Sauer, T.D, Yorke, J.A., Chaos - An Introduction to Dynamical Systems, Springer, 1994.

Anderson, T.J., Nayfeh, A.H., Balachandra, B., Experimental Verification of the Importance of the Nonlinear Curvature in the Response of a Cantilever Beam, Journal of Vibration and Acoustics, vol. 118 (21), pp. 21-27, 1996.

Andrade, V.S., Análise de Dinâmica Caótica de Pêndulos com Vibrações no Suporte, Escola de Engenharia de São Carlos, Dissertação de Mestrado, 2003.

Arfken, G.B., Mathematical Methods for Physicists, San Diego: Academic Press, 1995.

Argyris, J., Faust, G., Haase, M., An Exploration of Chaos, North-Holland, 1994.

Balthazar, J. M., Mook, D. T., Weber, H. I., Reyolando, M. L. R. F., Fenili, A., Belato, D., Felix, J. L. P., An Overview on Non-Ideal Vibrations, Meccanica, vol. 38 (6), pp. 613 $621,2003$.

Beer, F. P., e Johnston, E. R., Resistência dos Materiais, 3. ${ }^{\circ}$ Ed., Makron Books, 1995.

Belato, D., Não Linearidades do Eletro-Pêndulo, Faculdade de Engenharia Mecânica, Unicamp, Dissertação de Mestrado, 1998. 
Belato, D., Weber, H.I., Balthazar, J.M., Mook, D.T., Chaotic Vibrations of a Non-Ideal Electro-Mechanical System, International Journal Solids and Structure, vol.38, pp. 6691706, 2001.

Berdichevsky, V.L., Kim, W.W., Özbek, A., Dynamical Potential For Non-Linear Vibrations of Cantilevered Beams, Journal of Sound and Vibration, vol.179 (1), pp.151164, 1995.

Bodger, W. K. Deceleration of an Unbalanced Rotor Through a Critical Speed, Journal of Engineering for Industry, pp. 582-586, 1967.

Bolla, M. R., Balthazar, J. M., Felix, J. L. P., Mook, D. T., On an Approximate Analytical Solution to a Nonlinear Vibrating Problem Excited by a Nonideal Motor, Nonlinear Dynamics, vol. 50, pp. 841-847, 2007.

Broomhead, D., King, G., Extracting Qualitative Dynamics from Experimental Data, Physica D, vol. 20 (2-3), pp. 217-236, 1896.

Butkov, E., Física Matemática, Guanabara Dois, 1988.

Capistrano, V. B., Análise de Séries Temporais de Variáveis Microclimatológicas Medidas em Sinop-MT Utilizando a Teoria da Complexidade, Universidade Federal de Mato Grosso, Dissertação de Mestrado, 2007.

Cartmel, M. P., The Equations of Motion for a Parametrically Excited Cantilever Beam. Journal of Sound Vibration, vol. 143, n. 3, pp. 395-406, 1990.

Claeyssen, J.R., Copetti, R.D., Balthazar, J.M., Modal Analysis of a Beam with a Tip Rotor by Using a Fundamental Response, Materials Science Forum, vol. 440-441, pp. 261-268, 2003. 
Conrad, F., Morgül, Ö., On the Stabilization of a Flexible Beam with a Tip Mass, SIAM Journal on Control and Optimization, vol.36 (6), pp. 1962-1986, 1998.

Craig Jr, R., Structural Dynamics: An Introduction to Computer Methods, Wiley \& Sons, New York, 1981.

Crespo da Silva, M. R. M., Hodges, D. H., Nonlinear Flexure and Torsion of Rotating Beams, with Application to Helicopter Rotor Blades. I: Formulation, Vertica, vol. 10, pp. 151-169, 1986.

Crespo da Silva, M. R. M. and Hodges, D. H., Nonlinear Flexure and Torsion of Rotating Beams, with Application to Helicopter Rotor Blades. II: Response and Stability Results, Vertica, vol. 10, pp. 171-186, 1986.

Crespo da Silva, M. R. M., Non-linear Fflexural-Flexural-Torsional-Extensional Dynamics of Beams. I: Formulation, International Journal of Solids and Structures, vol. 24, pp. 1225-1234, 1988.

Crespo da Silva, M. R. M., Zaretzky, C. L., Hodges, D. H., Effects of Approxima-Tions on the Static and Dynamic Response of a Cantilever with a Tip Mass, International Journal of Solids and Structures, vol. 27, pp. 565-583, 1991.

Dantas, H. J. M., Balthazar, J. M., On the Appearance of a Hopf Bifurcation in a NonIdeal Mechanical Problem. Mechanics Research Communications, vol. 30, (5), pp. 493503, 2003.

da Nóbrega, P. G.B., Auto-Sincronização de Motores Não Ideais Apoiados em Estruturas Elásticas, Escola Politécnica da Universidade de São Paulo, Dissertação de Mestrado, 1994. 
de Mattos M. C., Balthazar, J. M., Dynamics of an Armature Controlled DC Motor Mounted on an Elastically supported Table, XV Congresso Brasileiro de Engenharia Mecânica, Águas de Lindóia, São Paulo, 1999.

de Mattos, M,C., Balthazar, J.M., Wieczorek, S., Mook, D.T., An Experimental Study of Vibrations of Nonideal Systems, Proceedings of DECT'97, DETC VIB-4011, ASME Design Engineering Technical Conferences, September 14-17, Sacramento, California, 1997.

de Souza, S.L.T., Caldas, I.L., Viana, R.L., Balthazar, J.M., Brasil, R.M.L.R.F., A Simple Feedback Control for a Chaotic Oscillator with Limited Power Supply, Journal of Sound and Vibration, vol. 299, pp. 664-671, 2007.

El-Badawy, A. A., Behavioral Investigation of a Nonlinear Nonideal Vibrating System, Journal of Vibration and Control, vol. 13(2), pp. 203-217, 2007.

Esmailzadeh, E., Jalili, N., Parametric Response of Cantilever Timoshenko Beams with Tip Mass Subjected to Harmonic Base Excitation, International Journal of Non-Linear Mechanics, vol. 33(5), pp. 765-781, 1998.

Felix, J. L. P., Teoria de Sistemas Vibratórios Aporticados Não Lineares e Não ideais, Faculdade de Engenharia Mecânica, Unicamp, Tese de Doutorado, 2002.

Fenili, A., Modelagem Matemática e Análise dos Comportamentos Ideal e Não-Ideal de Estruturas Flexíveis de Rastreamento com Aplicações em Engenharia Mecânica, Faculdade de Engenharia Mecânica, Unicamp, Tese de Doutorado, 2000.

Fenili, A., Balthazar, J.M., Brasil, R.M.L.F., On Mathematical Modeling of a Beam-Like Fexible Structure in Slewing Motion Assuming Nonlinear Curvature, Journal of Sound and Vibration vol. 268, pp. 825-838, 2003. 
Fenili, A., Balthazar, J.M., Some Remarks on Nonlinear Vibrations of Ideal and Nonideal Slewing Flexible Structures, Journal of Sound and Vibration, vol. 282 (1-2), pp. 543-552, 2005 .

Ferrara, N.F., Prado, C.P.C., Caos uma Introdução, Edgard Blucher, 1995.

Grassberger, P., Procaccia, I, Measuring the Strangeness of Strange Attractors, Physica D, vol. 9(1-2), pp. 186-192, 1983.

Gomes da Silva, D., Vibrações Não Lineares em Estruturas Ressonantes Tipo Viga, Escola de Engenharia de São Carlos, Tese de Doutorado, 2005.

Haddow, A. G., Mook, D. T., Barr, A. D. S., Theoretical and Experimental Study of Modal Interaction in a Two-Degree-of-Freedom Nonlinear Structure, Journal of Sound and Vibration, vol. 97 (3), pp. 451-473, 1984.

Hoa, S. V., Vibration of a Rotating Beam With Tip Mass, Journal os Sound and Vibration, vol. 67 (3), pp. 369-381, 1979.

Kantz, H., A Robust Method to Estimate the Maximal Lyapunov Exponent of a TimeSeries. Physics Letters A, vol. 185(1), pp. 77-87, 1994.

Kennel, M., Brown, R., Abarbanel, H., Determining Embedding Dimension from PhaseSpace Reconstruction Using a Geometrical Construction, Physical Review A, vol.25(6), pp. 3403-3411, 1992.

Kononenko, V. O., Vibrating Systems with a Limited Power Supply. London, London Iliffe, 1969. 
Krasnopolskaya, T.S., Shvets, A.Yu., Chaos in Vibrating Systems with a Limits PowerSupply Chaos, An Interdisciplinary Journal of Nonlinear Science, vol .3 (3), pp. 387-395, 1993.

Meirovitch, L., Methods of Analytical Dynamics. New York: Mcgraw-Hill, 1970;

Meirovitch, L., Elements of Vibrations Analysis. New York: Mcgraw-Hill, 1975;

Moon, F. C., Chaotic Vibrations an Introduction for Applied Scientists and Engineers. New York : Wiley, 1987.

Nayfeh, A.H, Mook, D.T., Nonlinear Oscillations. New York: Wiley \& Sons, 1979.

Nayfeh, A. H., Perturbation Methods. New York: Wiley \& Sons, 1973.

Nayfeh, A. H., Introduction to Perturbation Techniques. New York: Wiley \& Sons, 1981.

Nayfeh, A. H., Problems in Perturbation. New York: Wiley \& Sons, 1985.

Nayfeh, A.H., Balachandra, B., Applied Nonlinear Dynamics, Wiley, 1995.

Pai, P. F., Nayfeh, A. H., Three-Dimensional Nonlinear Vibrations of Composite Beams. I: Equations of Motion, Nonlinear Dynamics, vol. 1, pp. 477-502, 1990.

Palacios, J.L., Balthazar, J.M., Brasil, R. M. L. R. F., A Short Note on a Nonlinear System Vibrations Under Two Non-Ideal Excitations, Journal of the Brazilian Society of Mechanical Sciences and Engineering, vol. 25 (4), pp. 391-395, 2003. 
Palacios, J.L., Balthazar, J.M., Brasil, R. M. L. R. F., Comments on Nonlinear Dynamics of a Non-Ideal Duffing-Rayleigh Oscillator: Numerical and Analytical Approaches, Journal of Sound and Vibration, vol. 319, pp. 1136-1149, 2009.

Parker, T.S., Chua, L.O., Practical Numerical Algorithms for Chaotic Systems, Springer Verlag, 1989.

Piccirillo, V., Balthazar, J. M., Pontes B. R. J., Felix, J. L. P., On a Nonlinear and Chaotic Non ideal Vibrating System With Shape Memory Alloy(SMA), Journal of the Theoretical and Applied Mechanics, vol. 46, pp. 597-620, 2008.

Pontes, B. R. J., Oliveira, V. A., Balthazar, J. M., On Friction Driven Vibrations in a Mass Block-Belt-Motor System with a Limited Power Supply, Journal of Sound and Vibration, London, vol. 234 (4), pp. 713-723, 2000.

Pontes, B. R. J., Dinâmica e Controle de Sistemas Não-Lineares com Interação AutoExcitadora Sujeitos à Fontes de Energia do Tipo Ideal e Não-Ideal, Escola de Engenharia de São Carlos, Tese de Doutorado, 2003.

Pontes, B. R. J., Oliveira, V. A., Balthazar, J. M., On the Control of a Non-Ideal Engineering System: A Friction-Driven Oscillating System with Limited Power Supply, Materials Science Forum, Zurich, vol. 441 (1), pp. 355-362, 2003.

Rosenstein, M., Collins, J. J., Deluca, C. J., A Pratical Method for Calculating largest Lyapunov Exponents from Small Data Sets, Physica D, vol. 65 (1-2), pp. 117-134, 1993.

Savi, M. A., Caos em Sistemas Mecânicos, In: $1^{\circ}$ Congresso de Dinâmica, Controle e Aplicações da SBMAC, São José do Rio Preto - SP, 2002. 
Simo, J. C., A Finite Strain Beam Formulation. The Three Dimensional Dynamic Problem. Part I, Computer Methods in Applied Mechanics and Engineering, vol. 49 (1), pp. 55-70, 1985.

Simo, J. C., Vu-Quoc, L., On the Dynamics of Flexible Beams Under Large Overall Motions - The Plane Case: Part I, Journal of Applied Mechanics, vol. 53, pp. 849-854, $1986 \mathrm{a}$

Simo, J. C., Vu-Quoc, L., On the Dynamics of Flexible Beams Under Large Overall Motions - The Plane Case: Part II, Journal of Applied Mechanics, vol. 53, pp. 849-854, 1986b.

Simo, J. C., Vu-Quoc, L., The Role of Non Linear Theories in Transient Dynamic Analysis of Flexible Structures, Journal of Sound and Vibration, vol. 119 (3), pp. 487$508,1987$.

Simoni, A., R., Análise de Séries Temporais Aeroelásticas Experimentais Não Lineares, Escola de Engenharia de São Carlos, Tese de Doutorado, 2008.

Thompson, J. M. T., Stewart, H. B., Nonlinear Dynamics and Chaos. Wiley \& Sons, New York, 1986.

Timoshenko, S., History of Strength of Materials: With a Brief Account of the History of Theory of Elasticity and Theory of Structures. New York : McGraw-Hill, 1953.

Timoshenko, S., Young, D. H., Vibration Problems in Engineering. Princeton, 1955.

Timoshenko, S., Gere, J. M., Theory of Elastic Stability, 2 ed., McGraw-Hill, 1961. 
Timoshenko, S., Strength of Materials, Elementary Theory and Problems, Krieger Publishing Company, New York, 1995.

Tredinnick, M. R. A. C., Modelagem e Controle de Estruturas Espaciais Flexíveis, Instituto Nacional de Pesquisas Espaciais - INPE, Tese de Doutorado, 2006.

Tsuchida, M., Lolo, K. G., Balthazar, J.M., Silva, G.N., Cheshankov, B. I., On Regular and Irregular Vibrations of a Non-Ideal System with Two Degrees of Freedom. 1:1 Resonance, Journal of Sound and Vibration vol. 260, pp. 949-960, 2003.

Tsuchida, M., Guilherme, K. L., Balthazar, J. M., On Chaotic Vibrations of a Non-Ideal System with Two Degrees of Freedom: 1:2 Resonance and Sommerfeld Effect, Journal of Sound and Vibration, vol. 282, (3-5), pp. 1201-1207, 2005.

Yaman, M., Adomian Decomposition Method for Solving a Cantilever Beam of Varying Orientation with Tip Mass, Journal of computational and nonlinear dynamics, vol. 2, pp. $52-57,2007$.

Yamanaka, K., Heppler, G.R., Huseyin, K., On the Dynamics and Stability of a Beam with a Tip Rotor, AIAA Journal, vol. 35, pp. 1031-1038, 1994.

Williams, G., P., Chaos Theory Tamed, Taylor \& Francis Ltd, 1997.

Wolf, A., Swift, J.B., Swinney, H.L., Vastano, J.A., Determining Lyapunov Exponents From a Time Series, Physica vol. 16D, pp. 285-317, 1985.

Zavodney, L. D., Nayfeh, A. H., The Nonlinear Response of a Slender Beam Carrying a Lumped Mass to a Principal Parametric Excitation: Theory and Experiment. International Journal of nonlinear Mechanics, vol. 24 (2), pp. 105-125, 1989. 
Zhang, W., Chaotic Motion and its Control for Nonlinear Nonplanar Oscillations of a Parametrically Excited Cantilever Beam, Chaos, Solutions and Fractals, vol. 26, pp. 731$745,2005$.

Zukovic, M., Cveticanin, L., Chaotic Responses in a Stable Duffing System of Non-ideal Type, Journal of Vibration and Control, vol. 13 (6), pp. 751-767, 2007.

Zukovic, M., Cveticanin, L., Chaos in Non-ideal Mechanical System with Clearance, Journal of Vibration and Control, vol. 15 (8), pp. 1229-1246, 2009. 


\section{Apêndice A}

\section{Método de Múltiplas Escalas}

\section{A.1 Solução Aproximada para a Vibração Transversal Livre}

Para obter a solução do sistema representado pela Equação (3.1.23) utilize-se o método de múltiplas escalas como segue.

$\left\{\begin{array}{l}w(x, t, \beta)=w_{o}\left(x, T_{o}, T_{1}\right)+\beta w_{1}\left(x, T_{o}, T_{1}\right) \\ T_{o}=t \\ \mathrm{~T}_{1}=\beta t\end{array}\right.$

A equação do movimento para $\phi=0$ é dada por (Timoshenko, 1961), (Tredinnick, 2006).

$\frac{\partial^{4} w}{\partial x^{4}}-\beta \tilde{N}(t) \frac{\partial^{2} w}{\partial x^{2}}+\frac{\partial^{2} w}{\partial t^{2}}=0$

e

$$
\tilde{N}(t)=\left.R_{2} \frac{\partial^{3} w(x, t)}{\partial x \partial t^{2}}\right|_{x=L}
$$

onde $R_{2}=\frac{\beta^{2} E}{\rho L}$.

Substituindo (A1) em (A2) e mantendo termos até ordem $\beta$, obtém-se.

$$
\frac{\partial^{4} w_{o}}{\partial x^{4}}+\beta \frac{\partial^{4} w_{1}}{\partial x^{4}}+\beta \frac{\partial^{2} w_{o}}{\partial x^{2}} R_{2} \frac{\partial^{3} w_{o}}{\partial x \partial T_{o}{ }^{2}}+\frac{\partial^{2} w_{o}}{\partial T_{o}^{2}}+\beta\left\{2 \frac{\partial^{2} w_{o}}{\partial T_{o} \partial T_{1}}+\frac{\partial^{2} w_{1}}{\partial T_{o}^{2}}\right\}=0
$$

Para termos de ordem 0 tem-se.

$\frac{\partial^{4} w_{o}}{\partial x^{4}}+\beta \frac{\partial^{2} w_{o}}{\partial T_{o}^{2}}=0$ 
E para termos de ordem 1 tem-se.

$$
R_{2} \frac{\partial^{3} w_{o}}{\partial x \partial T_{o}^{2}} \frac{\partial^{2} w_{o}}{\partial x^{2}}+\frac{\partial^{4} w_{1}}{\partial x^{4}}+2 \frac{\partial^{2} w_{o}}{\partial T_{o} \partial T_{1}}+\frac{\partial^{2} w_{1}}{\partial T_{o}^{2}}=0
$$

A condição de contorno é dada por.

$$
\begin{aligned}
& \left\{\begin{array}{l}
w(0, t)=0 \\
\left.\frac{\partial w}{\partial x}\right|_{x=0}=0
\end{array}\right. \\
& {\left[\frac{\partial^{3} w}{\partial x^{3}}-R_{1} C_{1} \frac{\partial^{3} w}{\partial x \partial t^{2}}-C_{1} \frac{\partial^{2} w}{\partial t^{2}}+\beta R_{2} C_{1}\left(\frac{\partial^{2} w}{\partial x \partial t}\right)^{2}\right]_{x=1}=0} \\
& {\left[\frac{\partial^{2} w}{\partial x^{2}}+R_{1} \frac{\partial^{3} w}{\partial x^{3}}+C_{1} R_{2}^{2} \frac{\partial^{3} w}{\partial x \partial t^{2}}\right]_{x=1}=R_{2} C_{1} \beta\left[\frac{\partial w}{\partial x} \frac{\partial^{2} w}{\partial t^{2}}+\frac{\partial}{\partial x}\left(\frac{\partial^{3} w}{\partial x^{3}} \frac{\partial w}{\partial x}\right)-R_{1}\left(\frac{\partial^{2} w}{\partial x \partial t}\right)^{2}\right.}
\end{aligned}
$$

Utilizam-se as condições que determinam o engaste para obter a relação abaixo.

$w_{o}\left(0, T_{o}, T_{1}\right)+\beta w_{1}\left(0, T_{o}, T_{1}\right)=0$

então

$$
\left\{\begin{array}{l}
w_{o}\left(0, T_{o}, T_{1}\right)=0 \\
w_{1}\left(0, T_{o}, T_{1}\right)=0
\end{array}\right.
$$

Aplicando a condição de contorno em $x=0$ obtém-se.

$$
\left\{\begin{array}{l}
\left.\frac{\partial w_{o}}{\partial x}\right|_{x=0}=0 \\
\left.\frac{\partial w_{1}}{\partial x}\right|_{x=0}=0
\end{array}\right.
$$

Da mesma forma para $x=1$ tem-se.

$$
\begin{aligned}
& {\left[\frac{\partial^{3} w_{o}}{\partial x^{3}}+\beta \frac{\partial^{3} w_{1}}{\partial x^{3}}-R_{1} C_{1} \frac{\partial}{\partial x}\left\{\frac{\partial^{2} w_{o}}{\partial T_{o}^{2}}+\beta \frac{\partial^{2} w_{o}}{\partial T_{o} \partial T_{1}}+\beta\left[\frac{\partial^{2} w_{o}}{\partial T_{o} \partial T_{1}}+\frac{\partial^{2} w_{1}}{\partial T_{o}^{2}}\right]\right\}+\right.} \\
& \beta R_{2} C_{1}\left[\frac{\partial}{\partial x}\left\{\frac{\partial w_{o}}{\partial T_{o}}+\beta\left(\frac{\partial w_{o}}{\partial T_{1}}+\frac{\partial w_{1}}{\partial T_{o}}\right)\right]^{2}-C_{1}\left\{\frac{\partial^{2} w_{o}}{\partial T_{o}^{2}}+\beta \frac{\partial w_{o}}{\partial T_{o} \partial T_{1}}+\beta\left[\frac{\partial^{2} w_{o}}{\partial T_{o} \partial T_{1}}+\frac{\partial^{2} w_{1}}{\partial T_{o}^{2}}\right]\right]_{x=1}=0\right.
\end{aligned}
$$

Organizam-se os termos como segue.

$$
\begin{aligned}
& {\left[\frac{\partial^{3} w_{o}}{\partial x^{3}}+\beta \frac{\partial^{3} w_{1}}{\partial x^{3}}-R_{1} C_{1} \frac{\partial^{3} w_{o}}{\partial x \partial T_{o}^{2}}-\beta R_{1} C_{1} \frac{\partial^{3} w_{o}}{\partial x \partial T_{o} \partial T_{1}}-\beta R_{1} C_{1} \frac{\partial}{\partial x}\left[\frac{\partial^{2} w_{o}}{\partial T_{o} \partial T_{1}}+\frac{\partial^{2} w_{1}}{\partial T_{o}^{2}}\right]-\right.} \\
& \left.C_{1} \frac{\partial^{2} w_{o}}{\partial T_{o}^{2}}-C_{1} \beta \frac{\partial^{2} w_{o}}{\partial T_{o} \partial T_{1}}-C_{1} \beta\left[\frac{\partial^{2} w_{o}}{\partial T_{o} \partial T_{1}}+\frac{\partial^{2} w_{1}}{\partial T_{o}^{2}}\right]+\beta R_{2} C_{1} \frac{\partial^{2} w_{o}}{\partial x \partial T_{o}}\right]_{x=1}=0
\end{aligned}
$$


Utiliza-se (A10) para obter as relações abaixo.

$\left[\frac{\partial^{3} w_{o}}{\partial x^{3}}-R_{1} C_{1} \frac{\partial^{3} w_{o}}{\partial x \partial T_{o}^{2}}-C_{1} \frac{\partial^{2} w_{o}}{\partial T_{o}^{2}}\right]_{x=1}=0$

e

$\left\{\left[\frac{\partial^{3} w_{1}}{\partial x^{3}}-R_{1} C_{1}\left[2 \frac{\partial^{3} w_{o}}{\partial x \partial T_{o} \partial T_{1}}+\frac{\partial^{3} w_{1}}{\partial x \partial T_{o}^{2}}\right]+R_{2} C_{1} \frac{\partial^{2} w_{o}}{\partial x \partial T_{o}}-\right.\right.$

$\left.C_{1}\left[2 \frac{\partial^{2} w_{o}}{\partial T_{o} \partial T_{1}}+\frac{\partial^{2} w_{1}}{\partial T_{o}^{2}}\right]\right\}_{x=1}=0$

Desenvolvem-se os cálculos como segue.

$\left[\frac{\partial^{2} w_{o}}{\partial x^{2}}+\beta \frac{\partial w_{1}}{\partial x^{2}}+R_{1} \frac{\partial^{3} w_{o}}{\partial x^{3}}+\beta R_{1} \frac{\partial^{3} w_{1}}{\partial x^{3}}+\right.$

$\left.C_{1} R_{2}^{2} \frac{\partial}{\partial x}\left\{\frac{\partial^{2} w_{o}}{\partial T_{o}^{2}}+\beta \frac{\partial^{2} w_{o}}{\partial T_{o} \partial T_{1}}+\beta\left[\frac{\partial^{2} w_{o}}{\partial T_{o} \partial T_{1}}+\frac{\partial^{2} w_{1}}{\partial T_{o}^{2}}\right]\right\}\right]_{x=1}=$

$R_{2} C_{1} \beta\left[\frac{\partial w_{o}}{\partial x} \frac{\partial^{2} w_{o}}{\partial T_{o}^{2}}+\frac{\partial}{\partial x}\left(\frac{\partial^{3} w_{o}}{\partial x^{3}} \frac{\partial w_{o}}{\partial x}\right)-R_{1} \frac{\partial}{\partial x}\left(\frac{\partial w_{o}}{\partial T_{o}}\right)\right]$

então

$\left[\frac{\partial^{2} w_{o}}{\partial x^{2}}+R_{1} \frac{\partial^{3} w_{o}}{\partial x^{3}}+C_{1} R_{2}^{2} \frac{\partial^{3} w_{o}}{\partial x \partial T_{o}^{2}}\right]=0$

$\left[\frac{\partial^{2} w_{1}}{\partial x^{2}}+R_{1} \frac{\partial^{3} w_{1}}{\partial x^{3}}+C_{1} R_{2}^{2} \frac{\partial}{\partial x}\left[2 \frac{\partial^{2} w_{o}}{\partial T_{o} \partial T_{1}}+\frac{\partial^{2} w_{1}}{\partial T_{o}^{2}}\right]\right]_{x=1}=$

$R_{2} C_{1}\left[\frac{\partial w_{o}}{\partial x} \frac{\partial^{2} w_{o}}{\partial T_{o}^{2}}+\frac{\partial}{\partial x}\left(\frac{\partial^{3} w_{o}}{\partial x^{3}} \frac{\partial w_{o}}{\partial x}\right)-R_{1} \frac{\partial^{2} w_{o}}{\partial T_{o} \partial x}\right]$

Assumindo-se.

$w_{o}\left(x, T_{o}, T_{1}\right)=\Psi(x) a\left(T_{o}, T_{1}\right)$

Substitui-se em (A4) como segue.

$\frac{\partial^{4} \Psi(x)}{\partial x^{4}} a+\Psi \frac{\partial^{2} a}{\partial T_{o}^{2}}=0$

Integra-se em $T_{o}$ como abaixo.

$w_{o}\left(x, T_{o}, T_{1}\right)=A\left(T_{1}\right) \Psi(x) e^{i \omega T_{o}}$ 
Obtendo-se.

$\frac{\partial^{4} \Psi(x)}{\partial x^{4}}-w^{2} \phi=0$

sendo que a solução de $\mathrm{A}(15)$ é dada por.

$\Psi_{i}(x)=A_{1} \cos \left(\lambda_{i} x\right)+A_{2} \sinh \left(\lambda_{i} x\right)+A_{3} \cosh \left(\lambda_{i} x\right)+A_{4} \sinh \left(\lambda_{i} x\right)$

onde $\lambda_{i}=\sqrt{\omega_{i}}$.

Das condições de contorno em $x=0$ obtém-se

$w_{o}\left(0, T_{o}, T_{1}\right)=0$

$\Psi(0) a\left(T_{o}, T_{1}\right)=0$

$\Psi(0)=0$.

onde

$A_{1}+A_{3}=0$.

e

$\left.\frac{\partial w_{o}}{\partial x}\right|_{x=0}=\left.0 \Rightarrow \Psi\left(T_{o}, T_{1}\right)\left(\frac{\partial \Psi}{\partial x}\right)\right|_{x=0}=\left.0 \therefore\left[\frac{\partial \Psi}{\partial x}\right]\right|_{x=0}$

Obtendo-se a relação que segue

$$
A_{2}+A_{4}=0
$$

então

$\Psi_{i}(x)=A_{1}\left(\cosh \left(\lambda_{i} x\right)-\cos \left(\lambda_{i} x\right)\right)+A_{2}\left(\sinh \left(\lambda_{i} x\right)-\sin \left(\lambda_{i} x\right)\right)$

Substituindo-se nas condições de contorno em $x=1$ tem-se

$\left[\frac{\partial^{3} \Psi}{\partial x^{3}} a-R_{1} C_{1} \frac{\partial^{3}(\Psi a)}{\partial x \partial T_{o}^{2}}-C_{1} \frac{\partial^{2}(\Psi a)}{\partial T_{o}}\right]_{x=1}=0$

desta forma obtém-se a relação como segue

$$
A_{1}=-\frac{F_{2}}{F_{1}} A_{2}
$$

onde

$$
\begin{aligned}
& F_{1}=\lambda_{i}\left(\sinh \left(\lambda_{i}\right)-\sin \left(\lambda_{i}\right)\right)\left[\lambda_{i}^{2}+\omega^{2} R_{1} C_{1}\right]+C_{1}\left(\cosh \left(\lambda_{i}\right)-\cos \left(\lambda_{i}\right)\right) \\
& F_{2}=\lambda_{i}\left(\cosh \left(\lambda_{i}\right)-\cos \left(\lambda_{i}\right) \omega^{2} R_{1} C_{1}\right)+\lambda_{i}^{3}\left(\cosh \left(\lambda_{i}\right)+\cos \left(\lambda_{i}\right)\right)+C_{1}\left(\sinh \left(\lambda_{i}\right)-\sin \left(\lambda_{i}\right)\right)
\end{aligned}
$$


A figura abaixo mostra os três primeiros modos de vibrar da viga dada por (A16) (Fenili, 2000).

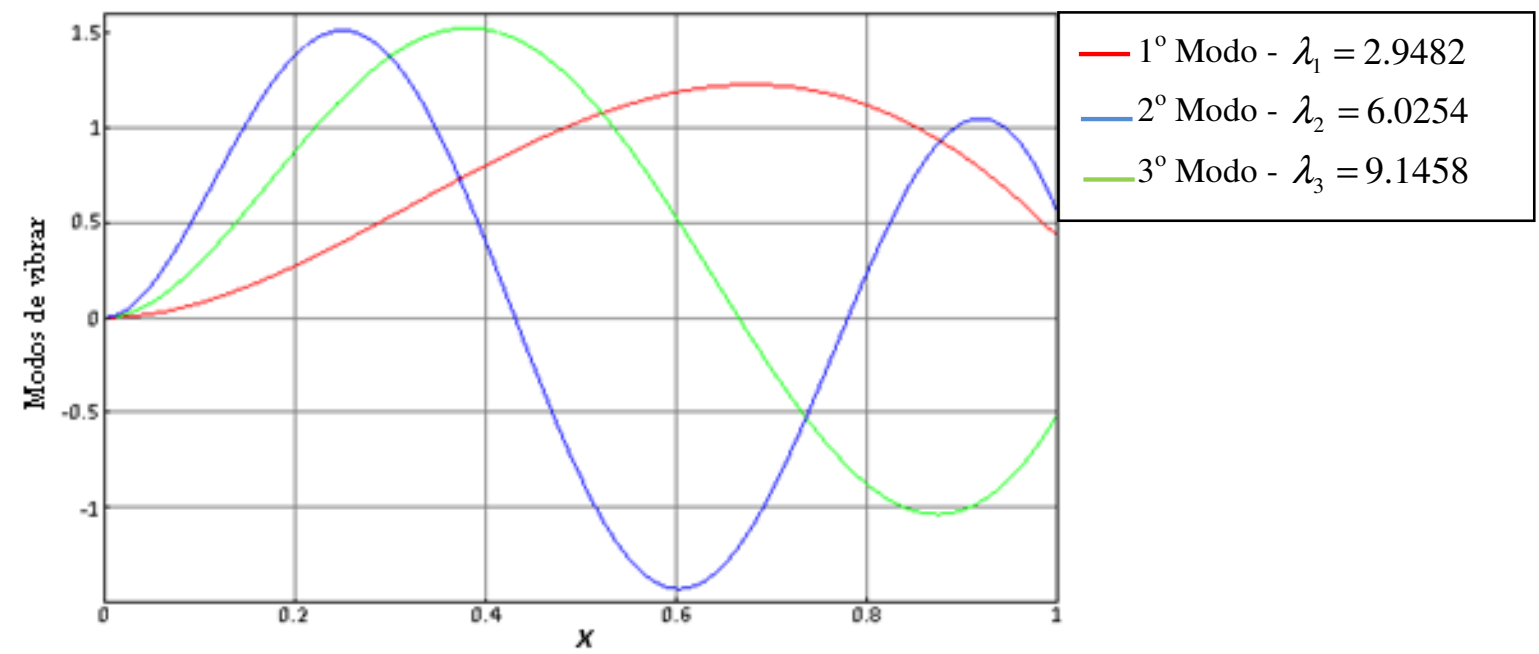

Figura A1 - Os três primeiros modos de vibrar da viga sem amortecimento e livre de esforços externos. 


\section{Apêndice B}

\section{Desenvolvimento dos Cálculos \\ Utilizando o Método dos Modos Assumidos nas \\ Condições de Contorno}

As duas condições de contorno que determinam o engaste da viga são dadas por

$\left\{\begin{array}{l}w(0, t)=0 \\ \left.\frac{\partial w(x, t)}{\partial x}\right|_{x=0}=0\end{array}\right.$

A equação que determina a terceira condição de contorno, devido à ação das forças no sistema, é reescrita como segue.

$\tilde{N} \sin \theta+\tilde{V} \cos \theta=\left[m_{R B} \vec{a}_{G}+m_{e} \vec{a}_{e}\right] \cdot \vec{j}$

Substituindo as funções e os termos adimensionais, temos:

$$
\begin{aligned}
& {\left[\frac{\beta E I}{L^{2}}\left\{\frac{\partial^{3} w}{\partial x^{3}}-\beta^{2} \frac{3}{2}\left(\frac{\partial w}{\partial x}\right)^{2}\right\}\left(1-\frac{\beta^{2}}{2}\left(\frac{\partial w}{\partial x}\right)^{2}\right)\right]-\left[e R _ { 1 } m _ { e } \left\{\frac{d^{2} \phi}{d t^{2}}\left(\beta \cos \phi \frac{\partial w}{\partial x}+\operatorname{sen} \phi\right)-\right.\right.} \\
& \left.\left.\left(\frac{d \phi}{d t}\right)^{2}\left[\cos \phi+\beta \operatorname{sen} \phi \frac{\partial w}{\partial x}\right]\right\}+R_{2} \frac{\partial^{3} w}{\partial x \partial t^{2}}\left\{M d_{2}+m_{e} L P_{2}\right\}\right] \beta \frac{\partial w}{\partial x}-\frac{\beta^{3}}{2}\left(\frac{\partial w}{\partial x}\right)^{3}= \\
& {\left[m_{R B} \vec{a}_{g}+m_{e} \vec{a}_{e}\right] j}
\end{aligned}
$$

Aplicando o método dos modos assumidos em (B2) temos a terceira condição de contorno, como segue.

$w(x, t)=\sum_{i} \Psi_{i}(x) q_{i}(t)$ 

de Contorno.

$$
\begin{aligned}
& {\left[\frac{\beta E I}{L^{2}}\left\{\sum_{i} \Psi_{i}^{\prime \prime \prime} q_{i}-\beta^{2} \frac{3}{2} \frac{d}{d x}\left[\left(\sum_{i} \Psi_{i}^{\prime} q_{i}\right)\left(\sum_{j} \Psi_{j}^{\prime} q_{j}\right)\right]\right\}\left(1-\frac{\beta^{2}}{2}\left(\sum_{p} \Psi_{p}^{\prime} q_{p}\right)\left(\sum_{r} \Psi_{r}^{\prime} q_{r}\right)\right)\right]-} \\
& {\left[e R_{1} m_{e}\left\{\ddot{\phi}\left(\beta \cos \phi \sum_{i} \Psi_{i}^{\prime} q_{i}+\operatorname{sen} \phi\right)-\dot{\phi}^{2}\left[\cos \phi+\beta \operatorname{sen} \phi \sum_{i} \Psi_{i}^{\prime} q_{i}\right]\right\}+\right.} \\
& \left.R_{2} \sum_{i} \Psi_{i}^{\prime} \ddot{q}_{i}\left\{M d_{2}+m_{e} L P_{2}\right\}\right] \beta \sum_{i} \Psi_{i}^{\prime} q_{i}-\frac{\beta^{3}}{2}\left[\left(\sum_{u} \Psi_{u}^{\prime} q_{u}\right)\left(\sum_{v} \Psi_{v}^{\prime} q_{v}\right)\left(\sum_{x} \Psi_{x}^{\prime} q_{x}\right)\right]= \\
& {\left[m_{R B} \vec{a}_{g}+m_{e} \vec{a}_{e}\right] j}
\end{aligned}
$$

Desenvolvendo os termos, obtemos.

$$
\begin{aligned}
& {\left[\frac{\beta E I}{L^{2}}\left\{\sum_{i} \Psi_{i}^{\prime \prime \prime} q_{i}-\beta^{2} \frac{3}{2}\left[\left(\sum_{i} \Psi_{i}^{\prime \prime} q_{i}\right)\left(\sum_{j} \Psi_{j}^{\prime} q_{j}\right)+\left(\sum_{i} \Psi_{i}^{\prime} q_{i}\right)\left(\sum_{i} \Psi_{j}^{\prime \prime} q_{j}\right)\right]\right\}\right.} \\
& \left.\left(1-\frac{\beta^{2}}{2}\left(\sum_{p} \Psi_{p}^{\prime} q_{p}\right)\left(\sum_{r} \Psi_{r}^{\prime} q_{r}\right)\right)\right]-\left[e R _ { 1 } m _ { e } \left\{\ddot{\phi}\left(\beta \cos \phi \sum_{i} \Psi_{i}^{\prime} q_{i}+\operatorname{sen} \phi\right)-\right.\right. \\
& \left.\left.\dot{\phi}^{2}\left[\cos \phi+\beta \operatorname{sen} \phi \sum_{i} \Psi_{i}^{\prime} q_{i}\right]\right\}+R_{2} \sum_{i} \Psi_{i}^{\prime} \ddot{q}_{i}\left\{M d_{2}+m_{e} L P_{2}\right\}\right] \beta \sum_{i} \Psi_{i}^{\prime} q_{i}- \\
& \frac{\beta^{3}}{2}\left[\left(\sum_{u} \Psi_{u}^{\prime} q_{u}\right)\left(\sum_{v} \Psi_{v}^{\prime} q_{v}\right)\left(\sum_{x} \Psi_{x}^{\prime} q_{x}\right)\right]=\left[m_{R B} \vec{a}_{g}+m_{e} \vec{a}_{e}\right] j
\end{aligned}
$$

A equação que determina a quarta condição de contorno, devido à ação dos momentos no sistema, é reescrita abaixo.

$$
\tilde{V}(\tilde{L}, \tilde{t}) d_{1}-\tilde{N}(\tilde{L}, \tilde{t}) d_{2}-\tilde{M}(\tilde{L}, \tilde{t})=\left(I_{R B}+I_{e}\right) \frac{\partial^{2} \theta(\tilde{L}, \tilde{t})}{\partial \tilde{t}^{2}}+I_{e} \frac{d^{2} \phi}{d t^{2}}
$$

Substituindo as funções em (B3) temos.

$$
\begin{aligned}
& \left(-\frac{\beta E I}{L^{2}}\left[\frac{\partial^{3} w}{\partial x^{3}}-\beta^{2} \frac{3}{2}\left(\frac{\partial w}{\partial x}\right)^{2}\right]\right) d_{1}-\left(e R _ { 1 } m _ { e } \left\{\frac{d^{2} \phi}{d t^{2}}\left[\beta \cos \phi \frac{\partial w}{\partial x}+\operatorname{sen} \phi\right]-\right.\right. \\
& \left.\left.\left(\frac{d \phi}{d t}\right)^{2}\left[\cos \phi+\beta \operatorname{sen} \phi \frac{\partial w}{\partial x}\right]\right\}+R_{2} \frac{\partial^{3} w}{\partial x \partial t^{2}}\left\{M d_{2}+m_{e} L P_{2}\right\}\right) d_{2}-\frac{E I}{L} \beta\left[\frac{\partial^{2} w}{\partial x^{2}}-\beta^{2} \frac{3}{2}\left(\frac{\partial w}{\partial x}\right)^{2}\right]= \\
& \left(I_{R B}+I_{e}\right) \frac{\partial^{2}}{\partial t^{2}}\left(\beta \frac{\partial w}{\partial x}-\frac{\beta^{3}}{2}\left(\frac{\partial w}{\partial x}\right)^{3}\right)+I_{e} \frac{d^{2} \phi}{d t^{2}}
\end{aligned}
$$

Aplicando o método dos modos assumidos em (B4) temos a quarta condição de contorno como segue. 


$$
\begin{aligned}
& \left(-\frac{\beta E I}{L^{2}}\left[\sum_{i} \Psi_{i}^{\prime \prime \prime} q_{i}-\beta^{2} \frac{3}{2}\left[\left(\sum_{i} \Psi_{i}^{\prime \prime} q_{i}\right)\left(\sum_{j} \Psi_{j}^{\prime} q_{j}\right)+\left(\sum_{i} \Psi_{i}^{\prime} q_{i}\right)\left(\sum_{i} \Psi_{j}^{\prime \prime} q_{j}\right)\right]\right]\right) d_{1}- \\
& \left(e R_{1} m_{e}\left\{\ddot{\phi}\left[\beta \cos \phi \sum_{i} \Psi_{i}^{\prime} q_{i}+\operatorname{sen} \phi\right]-\dot{\phi}^{2}\left[\cos \phi+\beta \operatorname{sen} \phi \sum_{i} \Psi_{i}^{\prime} q_{i}\right]\right\}+\right. \\
& \left.R_{2} \sum_{i} \Psi_{i}^{\prime} \ddot{q}_{i}\left\{M d_{2}+m_{e} L P_{2}\right\}\right) d_{2}-\frac{E I}{L} \beta\left[\sum_{i} \Psi_{i}^{\prime \prime} q_{i}-\beta^{2} \frac{3}{2}\left(\sum_{p} \Psi_{p}^{\prime} q_{p}\right)\left(\sum_{r} \Psi_{r}^{\prime} q_{r}\right)\right]= \\
& \left(I_{R B}+I_{e}\right) \frac{d^{2}}{d t^{2}}\left(\beta \sum_{i} \Psi_{i}^{\prime} q_{i}-\frac{\beta^{3}}{2}\left[\left(\sum_{u} \Psi_{u}^{\prime} q_{u}\right)\left(\sum_{v} \Psi_{v}^{\prime} q_{v}\right)\left(\sum_{x} \Psi_{x}^{\prime} q_{x}\right)\right]\right)+I_{e} \ddot{\phi}
\end{aligned}
$$

Desenvolvendo a derivada do termo depois da igualdade.

$$
\frac{d^{2}}{d t^{2}}\left(\beta \sum_{i} \Psi_{i}^{\prime} q_{i}-\frac{\beta^{3}}{2}\left[\left(\sum_{u} \Psi_{u}^{\prime} q_{u}\right)\left(\sum_{v} \Psi_{v}^{\prime} q_{v}\right)\left(\sum_{x} \Psi_{x}^{\prime} q_{x}\right)\right]\right)=
$$

Primeira derivada

$$
\begin{aligned}
& \frac{d}{d t}\left(\beta \sum_{i} \Psi_{i}^{\prime} \dot{q}_{i}-\left(\frac { \beta ^ { 3 } } { 2 } \left[\left(\sum_{u} \Psi_{u}^{\prime} \dot{q}_{u}\right)\left(\sum_{v} \Psi_{v}^{\prime} q_{v}\right)\left(\sum_{x} \Psi_{x}^{\prime} q_{x}\right)+\left(\sum_{u} \Psi_{u}^{\prime} q_{u}\right)\left(\sum_{v} \Psi_{v}^{\prime} \dot{q}_{v}\right)\left(\sum_{x} \Psi_{x}^{\prime} q_{x}\right)+\right.\right.\right. \\
& \left.\left.\left(\sum_{u} \Psi_{u}^{\prime} q_{u}\right)\left(\sum_{v} \Psi_{v}^{\prime} q_{v}\right)\left(\sum_{x} \Psi_{x}^{\prime} \dot{q}_{x}\right)\right]\right)
\end{aligned}
$$

Segunda derivada

$$
\begin{aligned}
& \beta \sum_{i} \Psi_{i}^{\prime} \ddot{q}_{i}-\frac{\beta^{3}}{2}\left[\left\{\left(\sum_{u} \Psi_{u}^{\prime} \ddot{q}_{u}\right)\left(\sum_{v} \Psi_{v}^{\prime} q_{v}\right)\left(\sum_{x} \Psi_{x}^{\prime} q_{x}\right)+\left(\sum_{u} \Psi_{u}^{\prime} \dot{q}_{u}\right)\left(\sum_{v} \Psi_{v}^{\prime} \dot{q}_{v}\right)\left(\sum_{x} \Psi_{x}^{\prime} q_{x}\right)+\right.\right. \\
& \left.\left(\sum_{u} \Psi_{u}^{\prime} \dot{q}_{u}\right)\left(\sum_{v} \Psi_{v}^{\prime} q_{v}\right)\left(\sum_{x} \Psi_{x}^{\prime} \dot{q}_{x}\right)\right\}+\left(\sum_{u} \Psi_{u}^{\prime} \dot{q}_{u}\right)\left(\sum_{v} \Psi_{v}^{\prime} \dot{q}_{v}\right)\left(\sum_{x} \Psi_{x}^{\prime} q_{x}\right)+\left(\sum_{u} \Psi_{u}^{\prime} q_{u}\right)\left(\sum_{v} \Psi_{v}^{\prime} \ddot{q}_{v}\right)\left(\sum_{x} \Psi_{x}^{\prime} q_{x}\right)+ \\
& \left(\sum_{u} \Psi_{u}^{\prime} q_{u}\right)\left(\sum_{v} \Psi_{v}^{\prime} \dot{q}_{v}\right)\left(\sum_{x} \Psi_{x}^{\prime} \dot{q}_{x}\right)+\left(\sum_{u} \Psi_{u}^{\prime} \dot{q}_{u}\right)\left(\sum_{v} \Psi_{v}^{\prime} q_{v}\right)\left(\sum_{x} \Psi_{x}^{\prime} \dot{q}_{x}\right)+\left(\sum_{u} \Psi_{u}^{\prime} q_{u}\right)\left(\sum_{v} \Psi_{v}^{\prime} \dot{q}_{v}\right)\left(\sum_{x} \Psi_{x}^{\prime} \dot{q}_{x}\right)+ \\
& \left(\sum_{u} \Psi_{u}^{\prime} q_{u}\right)\left(\sum_{v} \Psi_{v}^{\prime} q_{v}\right)\left(\sum_{x} \Psi_{x}^{\prime} \ddot{q}_{x}\right)=\left[{ }^{*}\right]
\end{aligned}
$$


Finalmente

$$
\begin{aligned}
& \left(-\frac{\beta E I}{L^{2}}\left[\sum_{i} \Psi_{i}^{\prime \prime \prime} q_{i}-\beta^{2} \frac{3}{2}\left[\left(\sum_{i} \Psi_{i}^{\prime \prime} q_{i}\right)\left(\sum_{j} \Psi_{j}^{\prime} q_{j}\right)+\left(\sum_{i} \Psi_{i}^{\prime} q_{i}\right)\left(\sum_{i} \Psi_{j}^{\prime \prime} q_{j}\right)\right]\right] d_{1}-\right. \\
& \left(e R_{1} m_{e}\left\{\ddot{\phi}\left[\beta \cos \phi \sum_{i} \Psi_{i}^{\prime} q_{i}+\operatorname{sen} \phi\right]-\dot{\phi}^{2}\left[\cos \phi+\beta \operatorname{sen} \phi \sum_{i} \Psi_{i}^{\prime} q_{i}\right]\right\}+\right. \\
& \left.R_{2} \sum_{i} \Psi_{i}^{\prime} \ddot{q}_{i}\left\{M d_{2}+m_{e} L P_{2}\right\}\right) d_{2}-\frac{E I}{L} \beta\left[\sum_{i} \Psi_{i}^{\prime \prime} q_{i}-\beta^{2} \frac{3}{2}\left(\sum_{p} \Psi_{p}^{\prime} q_{p}\right)\left(\sum_{r} \Psi_{r}^{\prime} q_{r}\right)\right]= \\
& \left(I_{R B}+I_{e}\right)[*]+I_{e} \ddot{\phi}
\end{aligned}
$$

onde $\gamma=e R_{1} m_{e}$

\section{B.1 Equações do Primeiro Modo de Vibrar}

\section{Equação da viga para $01^{0}$ modo}

$\ddot{q}\left(R_{2} q\left[M d_{2}+m_{e} L P_{2}\right]\left[G_{3}+G_{1}\right]-\beta^{2} E A\right)+q\left(\gamma G_{2} \ddot{\phi} \operatorname{sen} \phi-\dot{\phi}^{2} \gamma G_{2} \cos \phi+\beta \alpha G_{4}\right)+$
$q^{2}\left(\ddot{\phi} \gamma \beta^{2} \cos \phi\left[G_{3}+G_{1}\right]-\dot{\phi}^{2} \gamma \beta^{2} \operatorname{sen} \phi\left[G_{3}-G_{1}\right]-\beta^{3} \alpha G_{5}\right)-q^{3} \beta^{3} \alpha G_{6}=F \cos (\Omega t) G_{7}$

\section{Equação do motor}

$I_{e} \ddot{\phi}+I_{e} \ddot{q} \Psi^{\prime}=T-D$

\section{$3^{\mathrm{a}}$ Condição de contorno para $0 \mathbf{1}^{0}$ modo}

$$
\begin{aligned}
& {\left[\frac{\beta E I}{L^{2}}\left\{\Psi^{\prime \prime \prime} q-\beta^{2} \frac{3}{2}\left[2\left(\Psi^{\prime \prime} q\right)\left(\Psi^{\prime} q\right)\right]\right\}\left(1-\frac{\beta^{2}}{2}\left(\Psi^{\prime} q\right)^{2}\right)\right]-} \\
& {\left[e R_{1} m_{e}\left\{\ddot{\phi}\left(\beta \cos \phi\left(\Psi^{\prime} q\right)+\operatorname{sen} \phi\right)-\dot{\phi}^{2}\left[\cos \phi+\beta \operatorname{sen} \phi\left(\Psi^{\prime} q\right)\right]\right\}+\right.} \\
& \left.R_{2}\left(\Psi^{\prime}\right)^{2} \ddot{q}\left\{M d_{2}+m_{e} L P_{2}\right\}\right] \beta q-\frac{\beta^{3}}{2}\left[\left(\Psi^{\prime} q\right)^{3}\right]=\left[m_{R B} \vec{a}_{g}+m_{e} \vec{a}_{e}\right] j
\end{aligned}
$$

\section{$4^{\mathrm{a}}$ Condição de contorno para $01^{0}$ modo}

$$
\begin{aligned}
& \left(-\frac{\beta E I}{L^{2}}\left[\Psi^{\prime \prime \prime} q-\beta^{2} 3\left[\left(\Psi^{\prime \prime} q\right)\left(\Psi^{\prime} q\right)\right]\right) d_{1}-\left(e R_{1} m_{e}\left\{\ddot{\phi}\left[\beta \cos \phi\left(\Psi^{\prime} q\right)+\operatorname{sen} \phi\right]-\dot{\phi}^{2}\left[\cos \phi+\beta \operatorname{sen} \phi\left(\Psi^{\prime} q\right)\right]\right\}+\right.\right. \\
& \left.R_{2} \Psi^{\prime} \ddot{q}\left\{M d_{2}+m_{e} L P_{2}\right\}\right) d_{2}-\frac{E I}{L} \beta\left[\Psi^{\prime \prime} q-\beta^{2} \frac{3}{2}\left(\Psi^{\prime} q\right)^{2}\right]=\left(I_{R B}+I_{e}\right)[*]+I_{e} \ddot{\phi}
\end{aligned}
$$




\section{Apêndice C}

\section{Desenvolvimento dos Cálculos: Equação do Momento Fletor $\tilde{M}$}

A demonstração da Equação (3.1.5) do momento fletor descrita no Capítulo 3 é apresentada como segue.

Iniciam-se os cálculos, de acordo com a análise da figura abaixo.

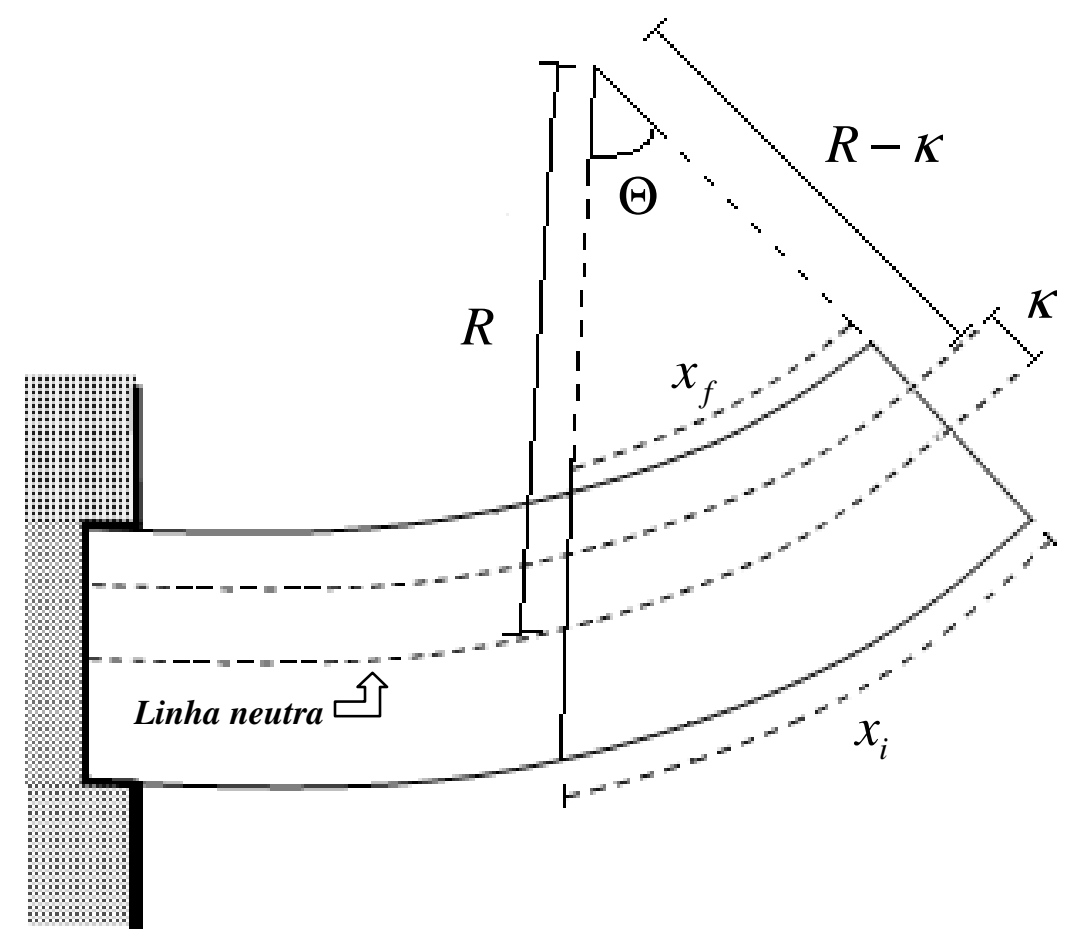

Figura C1 - Representação esquemática do elemento infinitesimal da viga para a obtenção da equação do momento. 
Onde:

$R=$ Raio de curvatura da viga.

$K=$ Deformação da viga acima da linha neutral.

$\Theta=$ Ângulo formado pelo prolongamento dos raios do elemento infinitesimal da viga.

$x_{i}=$ Deformação externa .

$x_{f}=$ Deformação interna .

A relação matemática entre o ângulo $\Theta$, as deformações $x_{i}$ e $x_{f}$ e o raio de curvatura $R$ juntamente com a deformação da viga $K$ é apresentada como segue.

$$
\Theta=\frac{x_{i}}{R}
$$

$\mathrm{e}$

$$
\Theta=\frac{x_{f}}{R-\kappa}
$$

Isolam-se as variáveis $x_{i}$ em (C1) e $x_{f}$ em (C2) como abaixo.

$$
x_{i}=R \Theta
$$

e

$$
x_{f}=\Theta(R-\kappa)
$$

A expressão da deformação é apresenta como segue.

$$
\xi_{x}=\frac{\Delta x}{x_{i}}=\frac{x_{f}-x_{i}}{x_{i}}
$$

Substituindo-se (C3) e (C4) em (C5), tem-se

$$
\xi_{x}=\frac{\Theta(R-\kappa)-R \Theta}{R \Theta}=-\frac{\kappa}{R}
$$

O sinal negativo indica que a deformação $K$ está na região de diminuição das fibras da viga (Beer, 1995). 
Isola-se $R$ em (C6) como segue

$$
R=-\frac{\kappa}{\xi_{x}}
$$

As expressões que representam a tensão na viga são mostradas abaixo.

$$
\sigma_{x}=\xi_{x} E
$$

onde $E$ é o módulo de elasticidade.

$\mathrm{ou}$

$$
\sigma_{x}=\frac{\sum F}{d A}
$$

onde $d A$ a área do elemento infinitesimal da viga e $\sum F$ as força que agem no elemento.

Isolando $\sum F$ em (C9) tem-se.

$\sum F=\sigma_{x} d A$

Substituindo-se (C6) em (C8) e depois em (C10) obtém-se

$$
\sum F=-\frac{E}{R} \int \kappa d A
$$

Onde o termo $\int \kappa d A$ é a definição do momento estático (Beer, 1995)

A equação do momento é mostrada com segue.

$M= \pm \sum F \cdot d$

sendo que, nesta análise, o deslocamento $d=\kappa$.

Substituindo-se (C11) em (C12) tem-se.

$M=\frac{E}{R} \int \kappa^{2} d A$

onde $\int \kappa^{2} d A$ é a definição do momento de inércia $(I)$.

Sendo assim, pode-se escrever (C13) como abaixo

$$
M=\frac{E I}{R}
$$

Define-se a expressão que representa a curvatura da viga $\left(\frac{1}{R}\right)$ como segue (Beer, 1995). 
$\left(\frac{1}{R}\right)=\frac{f^{\prime \prime}(x)}{\left[1+\left(f^{\prime}(x)\right)^{2}\right]^{\frac{3}{2}}}$

Substituindo-se (C15) em (C14) obtém-se.

$M=E I \cdot f^{\prime \prime}(x) \cdot\left[1+\left(f^{\prime}(x)\right)^{2}\right]^{-\frac{3}{2}}$

Reescreve-se (C16) na notação utilizada neste trabalho, de forma a obter a expressão para o momento fletor da viga, como segue.

$\tilde{M}=E I \frac{\partial^{2} \tilde{w}}{\partial \tilde{x}^{2}}\left[1+\left(\frac{\partial \tilde{w}}{\partial \tilde{x}}\right)^{2}\right]^{-\frac{3}{2}}$ 


\section{Apêndice D}

\section{Parâmetros Físicos do Motor}

O motor utilizado nos ensaios experimentais foi o EN35 M type $24 \mathrm{~V}$.

\section{Motors}
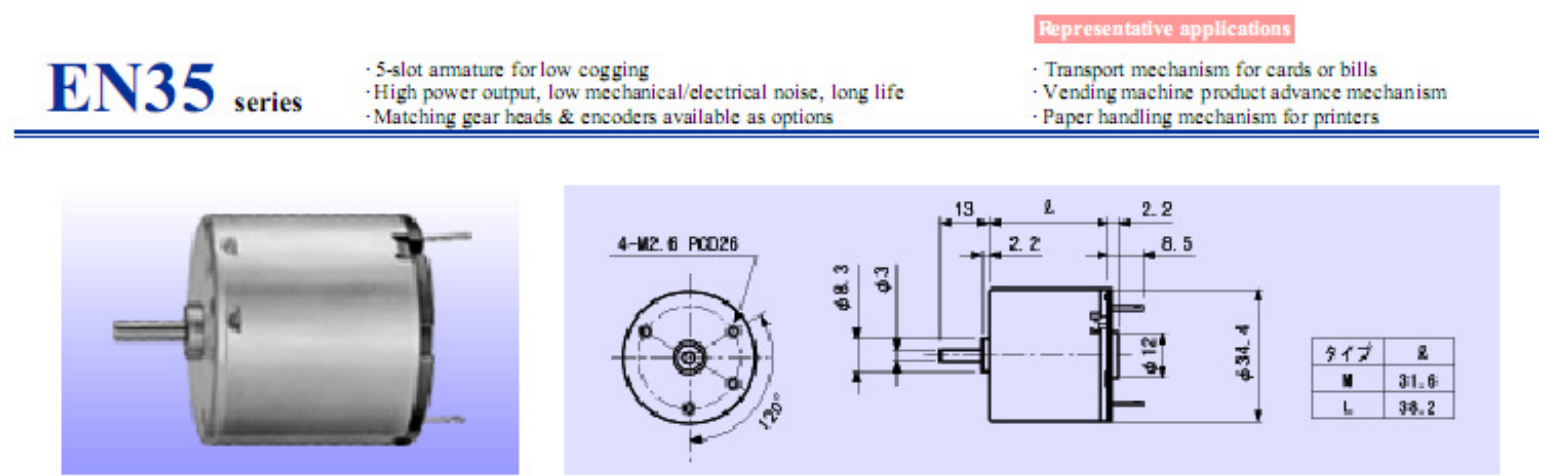

EN35 M type

EN35 L type

\begin{tabular}{|c|c|c|c|c|c|c|}
\hline & & & \multicolumn{2}{|c|}{ EN35 M type } & \multicolumn{2}{|c|}{ EN35 L type } \\
\hline \multicolumn{2}{|c|}{ Voltage } & V & 12 & 24 & 12 & 24 \\
\hline \multirow{5}{*}{$\begin{array}{l}\mathrm{R} \\
\mathrm{a} \\
\mathrm{t} \\
\mathrm{c} \\
\mathrm{d}\end{array}$} & Power output & W & 2.3 & 2.5 & 4.5 & 4.5 \\
\hline & \multirow[t]{2}{*}{ Torque } & $\mathrm{mN} \cdot \mathrm{m}$ & 3.92 & 3.92 & 7.85 & 7.85 \\
\hline & & g-cm & 40 & 40 & 80 & 80 \\
\hline & Speed & $\mathrm{r} / \mathrm{min}$ & 5,700 & 6,000 & 5,500 & 5,500 \\
\hline & Current & $\mathrm{mA}$ & 340 & 175 & 550 & 275 \\
\hline \multirow{2}{*}{$\begin{array}{l}\mathrm{N} \\
0 \\
1 \\
\mathrm{~d} \\
\mathrm{a} \\
\mathrm{d} \\
\end{array}$} & Speed & $\mathrm{r} / \mathrm{min}$ & 6,500 & 6,900 & 6,600 & 6,600 \\
\hline & Current & $\mathrm{mA}$ & 95 & 50 & 95 & 45 \\
\hline \multirow{2}{*}{\multicolumn{2}{|c|}{ Starting torque }} & $\mathrm{mN} \cdot \mathrm{m}$ & 31.4 & 29.4 & 46.1 & 49.0 \\
\hline & & g-cm & 320 & 300 & 470 & 500 \\
\hline \multirow{2}{*}{\multicolumn{2}{|c|}{ Torque constant }} & $\mathrm{mN} \cdot \mathrm{m} / \mathrm{A}$ & 16.1 & 30.8 & 17.1 & 33.7 \\
\hline & & $\mathrm{g}-\mathrm{cm} / \mathrm{A}$ & 164 & 314 & 174 & 344 \\
\hline
\end{tabular}

Fonte: http://www.tridenteng.co.uk/products.php?type=dc\&extra=Canon 


\section{Apêndice $\mathbf{E}$}

\section{Reconstrução do Espaço de Fase}

A presença de caos no experimento também pode ser investigada. Esta análise é feita através do estudo do sistema a partir de séries temporais, sendo que o principal método disponível é o da reconstrução do espaço de fase (Simoni, 2008).

Neste apêndice, apresenta-se uma síntese sobre a técnica de reconstrução do espaço de fase, sendo que esta técnica será utilizada para determinar a presença ou não do comportamento cáotico em experimentos.

Em um contexto geral, um experimento não mede todas as variáveis presentes no sistema e, usualmente, tem-se disponível a evolução no tempo de uma única variável. Sendo assim, é conveniente analisar o sistema dinâmico a partir de uma série temporal.

Os estudos iniciais para reconstruir o espaço de fase de um sistema por meio da utilização de medidas experimentais, foi através do uso de equações diferenciais, porém tais estudo mostraram que as derivadas do sinal obtido, que tinham como objetivo encontrar uma trajetória da órbita no espaço de fase, perdiam informações devido a uma aproximação numérica das soluções das derivadas (Thompson, 1986), (Alligood, 1996) e (Ferrara, 1995).

Um forma conveniente de reconstruir a dinâmica do sistema se dá a partir da obtenção de variáveis $\left\{X_{K}(t)\right\}$ (sendo $\mathrm{k}=1, \ldots, \mathrm{n}-1$ ), a partir de uma única série temporal $X_{0}(t)$. A técnica consiste em utilizar a série temporal original $X_{0}(t)$, obtida experimentalmente e, aplicar pequenos deslocamentos temporais sucessivos com uma defasagem fixa $\tau(\tau=\mathrm{m} \Delta \mathrm{t}$, onde $\mathrm{m}$ é um número inteiro e $\Delta \mathrm{t}$ é o tempo de amostragem do instrumento usado no experimento) para $\mathrm{N}$ pontos equidistantes do conjunto de dados, como mostrado na Equação E1 abaixo.

$$
\begin{aligned}
& X_{0}=X_{0}\left(t_{1}\right), \ldots, X_{0}\left(t_{N}\right) \\
& X_{1}=X_{0}\left(t_{1}+\tau\right), \ldots, X_{0}\left(t_{N}+\tau\right) \\
& \vdots \\
& X_{n-1}=X_{0}\left[\left(t_{1}+(n-1) \tau\right), \ldots, X_{0}\left(t_{N}+(n-1) \tau\right)\right]
\end{aligned}
$$


A aplicação do método da defasagem na série temporal não é suficiente para se fazer a reconstrução do espaço de fase. A escolha da dimensão de imersão e do tempo de defasagem são importantes nos casos dos conjuntos de dados experimentais onde existe a presença de ruídos.

Para determinar a dimensão mínima de imersão, encontram-se na literatura três métodos: método da saturação dos invariantes do sistema (Grassberger, 1983), método da decomposição em valores singulares (Broomhead, 1986) e o método das falsas vizinhanças (Kennel et al., 1992).

Para ilustrar o método da reconstrução de sistema por análise de séries temporais, apresenta-se um exemplo, sugerido por Otto Rössler (1976), que corresponde a um modelo matemático com comportamento caótico (Capistrano, 2007). O sistema de equações, conhecido como atrator de Rössler, é mostrado como segue.

$$
\begin{aligned}
& \frac{d x}{d t}=-y-z \\
& \frac{d y}{d t}=x+a y \\
& \frac{d z}{d t}=b+z(x-c)
\end{aligned}
$$

A Figura E1 ilustra o resultado das equações do modelo de Rössler no espaço de fase. Utiliza-se para a construção do atrator um número de amostragem de 10000 e dt $=0.01$, para $\mathrm{a}=0.32, \mathrm{~b}=0.3$ e $\mathrm{c}=4.5$, como segue.

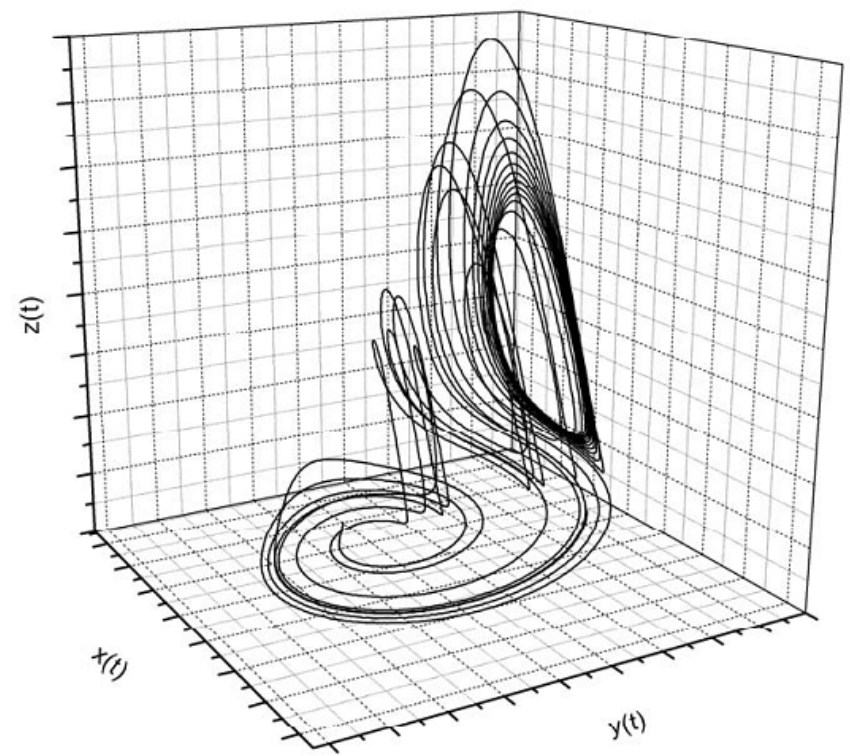

Figura E1 - Atrator caótico do modelo de Rössler (Alligood, 1996) e (Ferrara, 1995). 
A Figura E2 ilustra a série temporal $x(t)$, cujo resultado tem origem na solução do sistema representado pela Equação (E2).

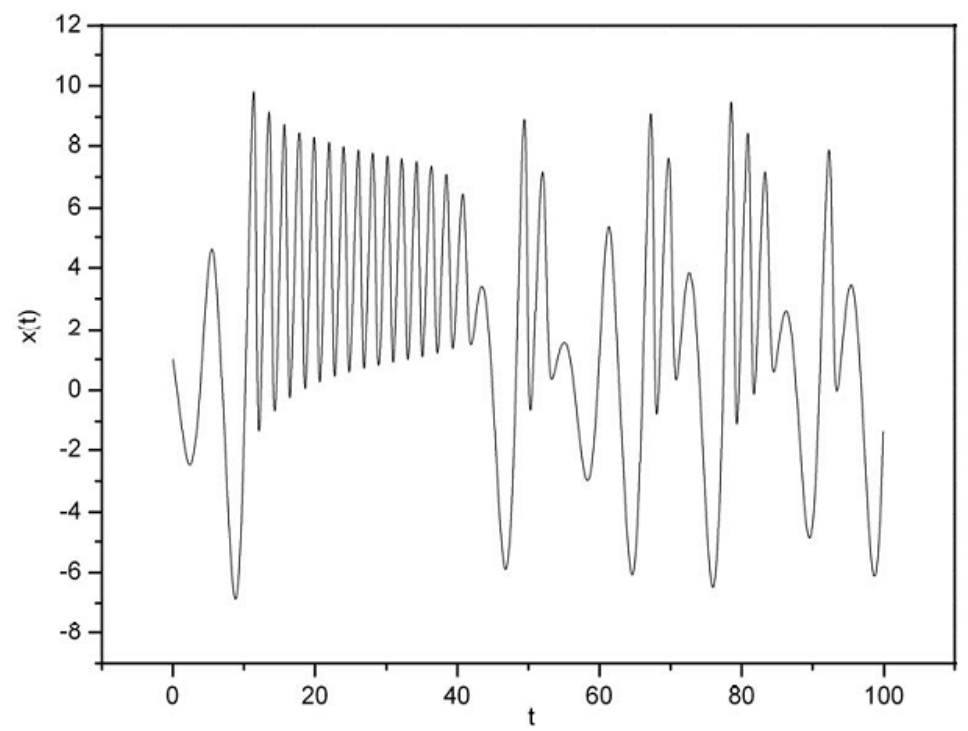

Figura E2 - Histórico no tempo do atrator de Rössler (Alligood, 1996) e (Ferrara, 1995).

A reconstrução do espaço de fase do atrator de Rössler é feita, através da utilização da série temporal mostrada na Figura E2 e utilizando-se o artifício da defasagem temporal apresenta-se a reconstrução do atrator na Figura E3, como segue.

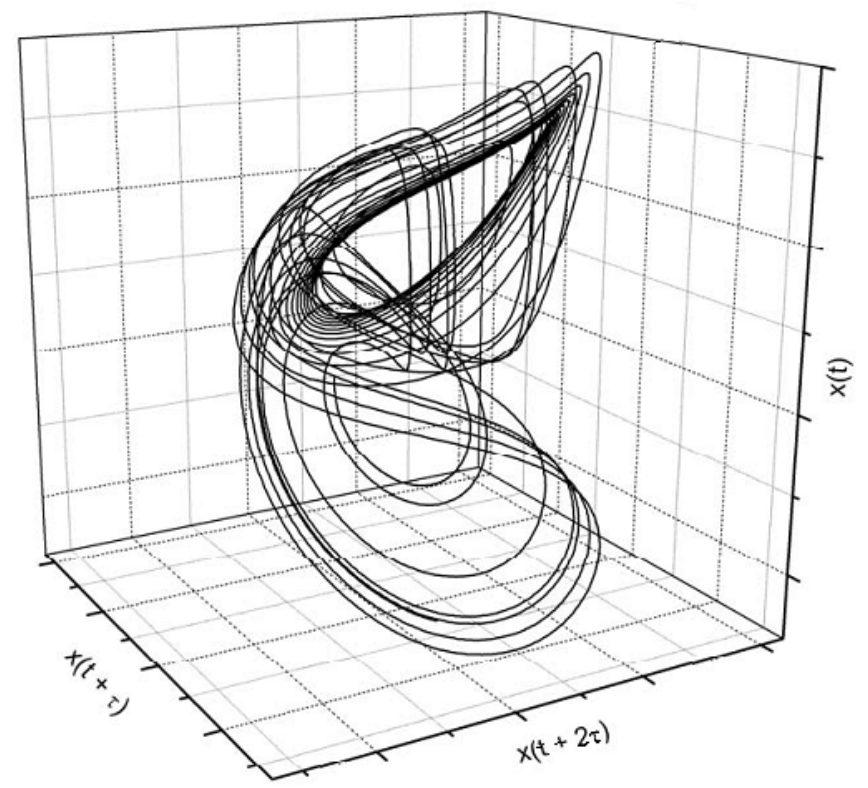

Figura E3 - Reconstrução do atrator de Rössler por meio da defasagem da série temporal x(t) (Alligood, 1996) (Ferrara, 1995). 
Compara-se a Figura E1 com a Figura E3, observa-se que a geometria e os traços do plano de fase dos gráficos são semelhantes. Porém os traços da Figura E3 se apresentam mais distorcidos, devido aos inevitáveis erros que surgem durante os ensaios. Desta forma, através da defasagem temporal foi possível reconstruir o espaço de fase do atrator de Rössler.

Sendo assim, conclui-se que o método para a reconstrução do espaço de fase se mostrou muito eficaz no exemplo apresentado.

A obtenção de caos no sistema é feita através da análise dos sinais dos expoentes de Lyapunov, onde avaliam a sensibilidade às condições iniciais, verificando a divergência exponencial no tempo de trajetórias vizinhas. Wolf et al. (1985) desenvolveu o primeiro algoritmo para calcular os expoentes de Lyapunov para séries temporais. Trabalhos mais recentes como Rosenstein et al. (1993), Kantz (1994) e Savi (2002), discutem outras formas da obtenção dos expoentes de Lyapunov utilizando séries temporais. 


\section{Apêndice F}

\section{Programas Utilizados nas Simulações Numéricas}

Neste apêndice são apresentados os códigos dos programas desenvolvidos na obtenção de gráficos apresentados no Capítulo 4, utilizando o software Matlab ${ }^{\circledR} 8$.

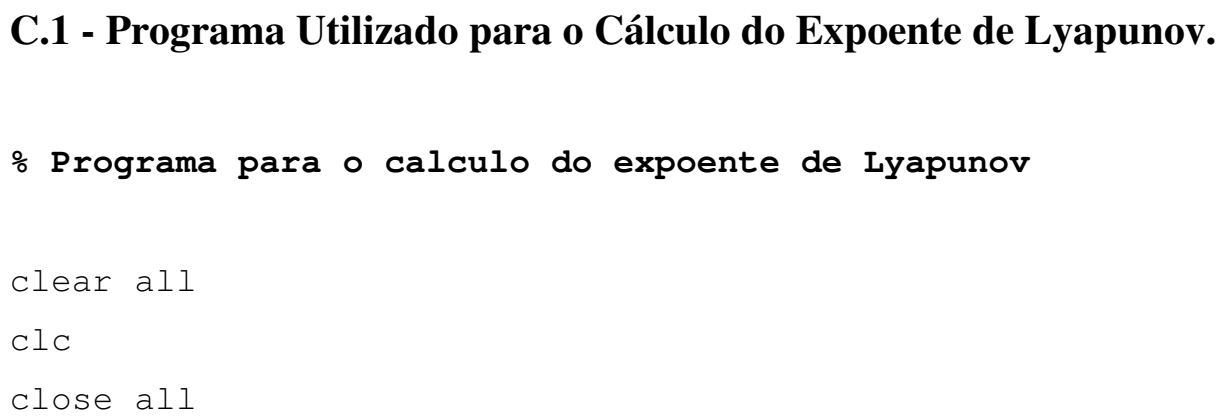


\% Condição inicial \%

$$
\mathrm{x} 0=\left[\begin{array}{llllll}
0.0001 & 0.0001 & 0.0001 & 0.0001 & 0.0001
\end{array}\right] \text {; }
$$

\section{\% Obtenção do vetor de estados do sistema \%}

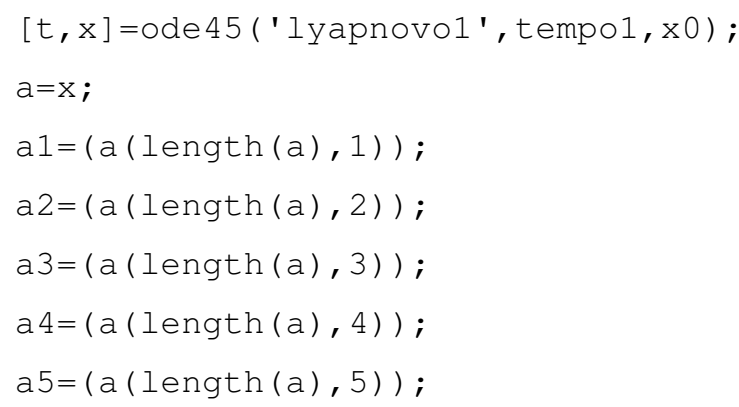

\% pontos de linearização a serem usados no sistema linearizado \% $\mathrm{xo}=\left[\begin{array}{lllll}\mathrm{a} 1 & \mathrm{a} 2 & \mathrm{a} 3 & \mathrm{a} 4 & \mathrm{a} 5\end{array}\right]^{\prime}$;

\% sistema linear

\% Intervalo de tempo \%

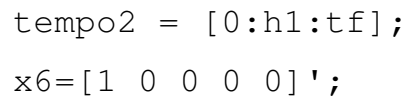

\% Obtenção do vetor de estados do sistema \%

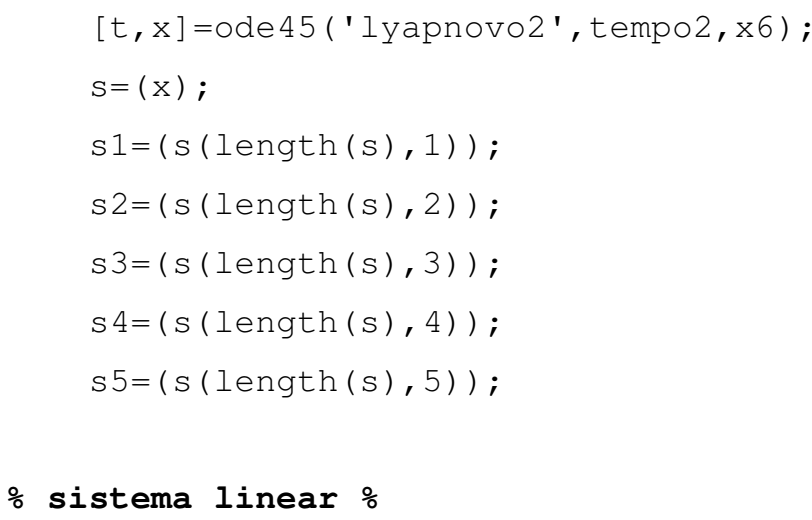


\% Obtenção do vetor de estados do sistema \%

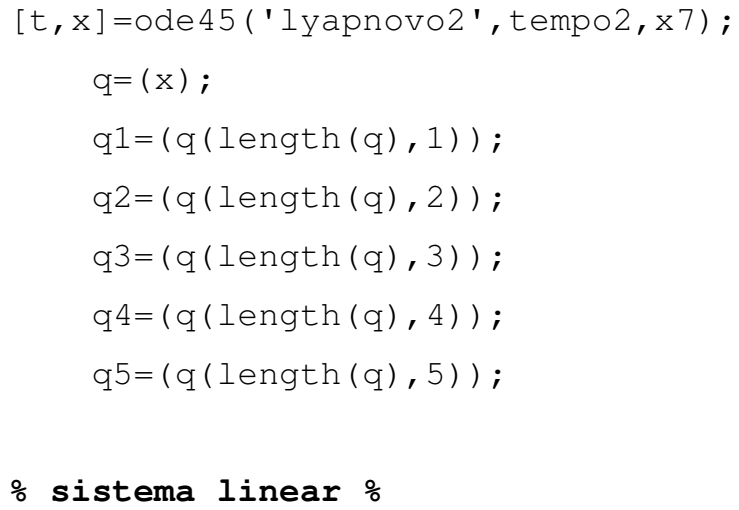

\% sistema linear $\%$

\% Obtenção do vetor de estados do sistema \%

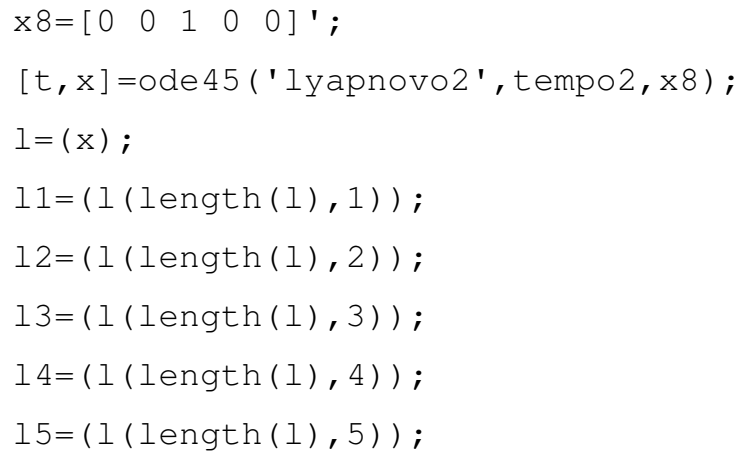

\% sistema linear \%

\% Obtenção do vetor de estados do sistema \%

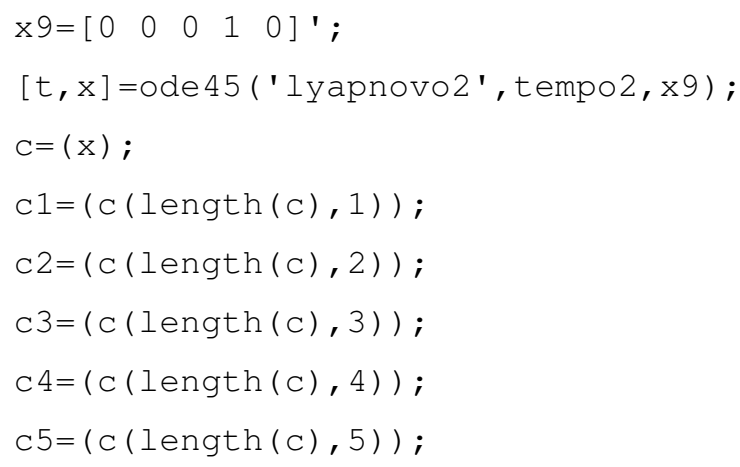

\% sistema linear \%

$\mathrm{x} 10=\left[\begin{array}{lllll}0 & 0 & 0 & 0 & 1\end{array}\right]^{\prime} ;$ 
\% Obtenção do vetor de estados do sistema \%

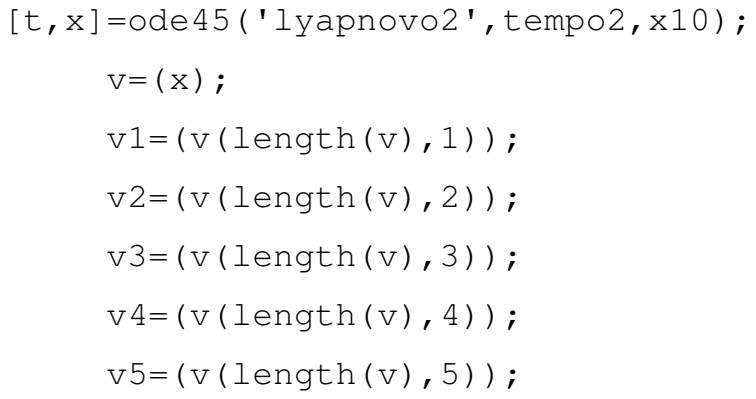

\% últimos valores de cada iteração \%

$$
\begin{aligned}
& \mathrm{z} 1=\left[\begin{array}{lllll}
\mathrm{a} 1 & \text { a2 } & \text { a3 } & \text { a4 } & \text { a5 }
\end{array}\right]^{\prime} ; \\
& \mathrm{y} 1=\left[\begin{array}{lllll}
\mathrm{s} 1 & \text { s2 } & \text { s3 } & \text { s4 } & \text { s5 }
\end{array}\right]^{\prime} ; \\
& \mathrm{y} 2=\left[\begin{array}{llllll}
\text { q1 } & \text { q2 } & \text { q3 } & \text { q4 } & \text { q5 }
\end{array}\right]^{\prime} ; \\
& \mathrm{y} 3=\left[\begin{array}{lllll}
11 & 12 & 13 & 14 & 15
\end{array}\right]^{\prime} ; \\
& \mathrm{y} 4=\left[\begin{array}{lllll}
\mathrm{c1} & \mathrm{c} 2 & \mathrm{c} 3 & \mathrm{c} 4 & \mathrm{c5}
\end{array}\right]^{\prime} ; \\
& \mathrm{y} 5=\left[\begin{array}{lllll}
\mathrm{v} 1 & \mathrm{v} 2 & \mathrm{v} 3 & \mathrm{v} 4 & \mathrm{v} 5
\end{array}\right]^{\prime} ;
\end{aligned}
$$

\% passo mais preciso \%

hh=0.00001;

for $i=1: 40000$

\% ortonormalização \%

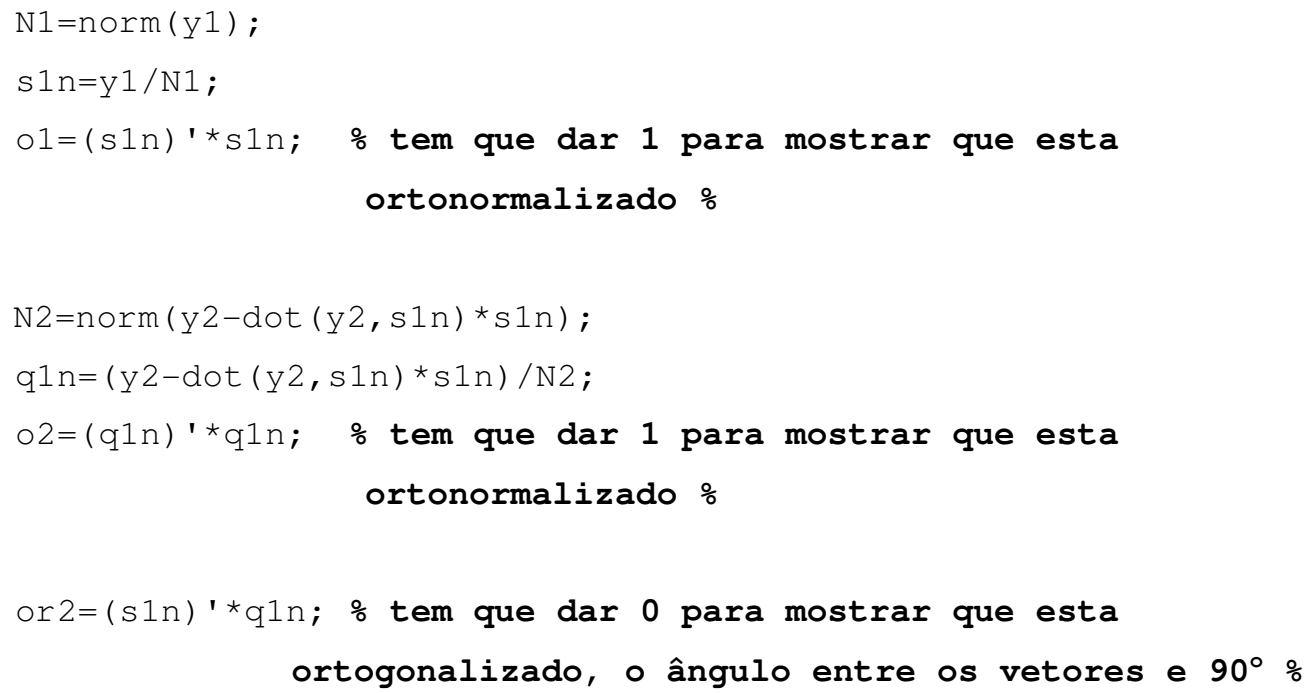




$$
\begin{aligned}
& 12=(1(1 \operatorname{ength}(1), 2)) ; \\
& 13=(1(1 \operatorname{ength}(1), 3)) ; \\
& 14=(1(1 \operatorname{ength}(1), 4)) ; \\
& 15=(1(\operatorname{length}(1), 5)) ;
\end{aligned}
$$

\% sistema linear \%

\% Obtenção do vetor de estados do sistema \%

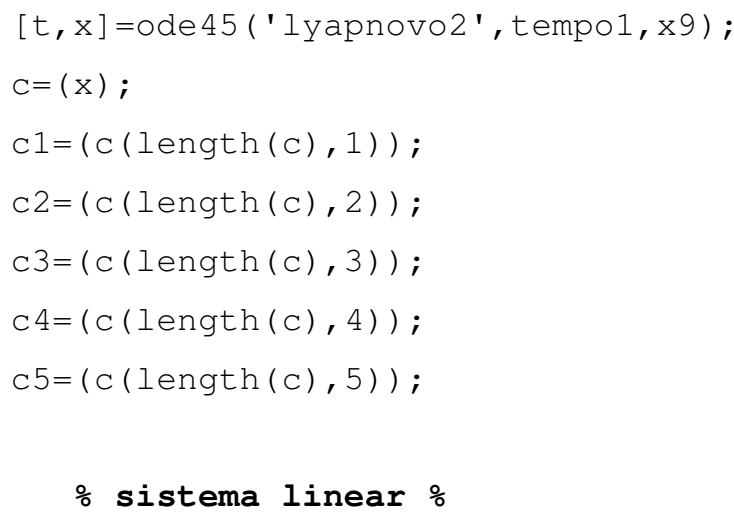

\% Obtenção do vetor de estados do sistema \%

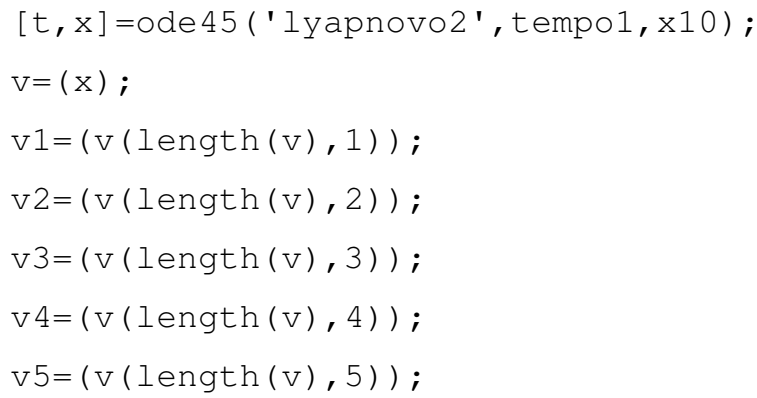

\% nova CI para a próxima iteração, guarda os valores das últimas iterações e volta no inicio do for com esses novos valores \%

$$
\begin{aligned}
& \mathrm{z} 1=\left[\begin{array}{lllll}
\mathrm{a} 1 & \text { a2 } & \text { a3 } & \text { a4 } & \text { a5 }
\end{array}\right]^{\prime} \text {; } \\
& \mathrm{y} 1=\left[\begin{array}{lllll}
\mathrm{s} 1 & \mathrm{~s} 2 & \mathrm{~s} 3 & \mathrm{~s} 4 & \mathrm{~s} 5
\end{array}\right]^{\prime} \text {; } \\
& \mathrm{y} 2=\left[\begin{array}{lllll}
q 1 & q 2 & \text { q3 } & \text { q4 } & \text { q5 }
\end{array}\right]^{\prime} \text {; } \\
& \mathrm{y} 3=\left[\begin{array}{lllll}
11 & 12 & 13 & 14 & 15
\end{array}\right]^{\prime} \text {; } \\
& \mathrm{y} 4=\left[\begin{array}{lllll}
\mathrm{c} 1 & \mathrm{c} 2 & \mathrm{c} 3 & \mathrm{c} 4 & \mathrm{c} 5
\end{array}\right]^{\prime} \text {; } \\
& \mathrm{y} 5=\left[\begin{array}{lllll}
\mathrm{v} 1 & \mathrm{v} 2 & \mathrm{v} 3 & \mathrm{v} 4 & \mathrm{v} 5
\end{array}\right]^{\prime} \text {; }
\end{aligned}
$$




\section{\%Fazendo o somatório \%}

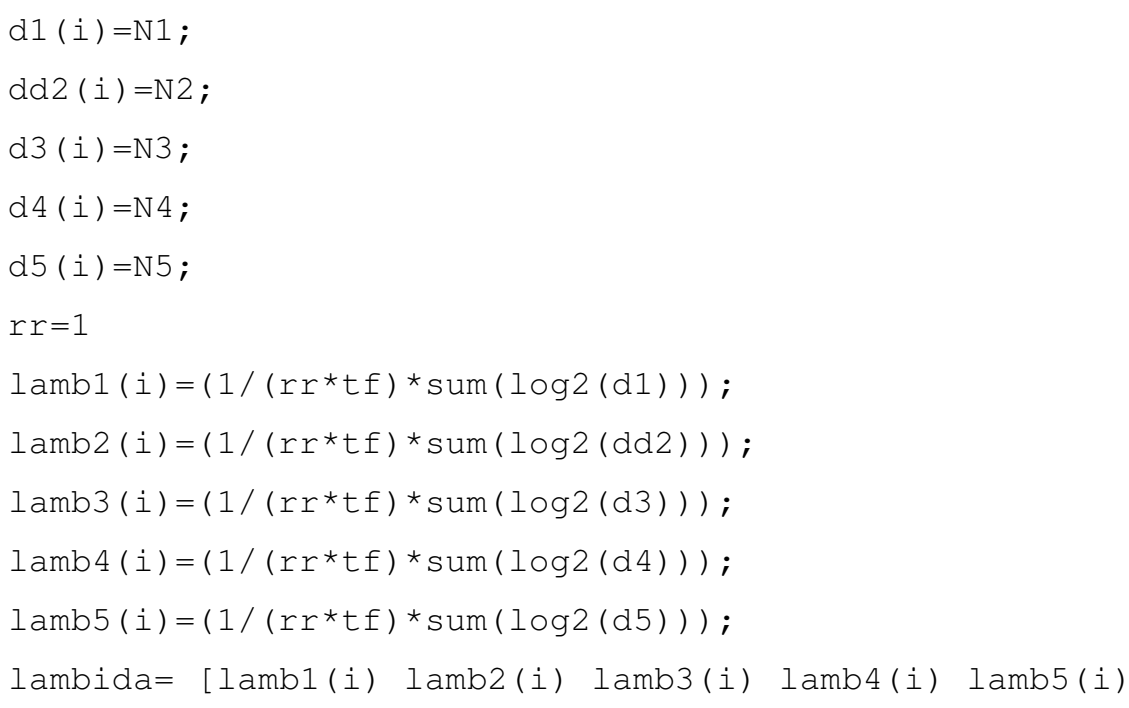

\section{\% Plotagem dos resultados $\frac{\%}{0}$}

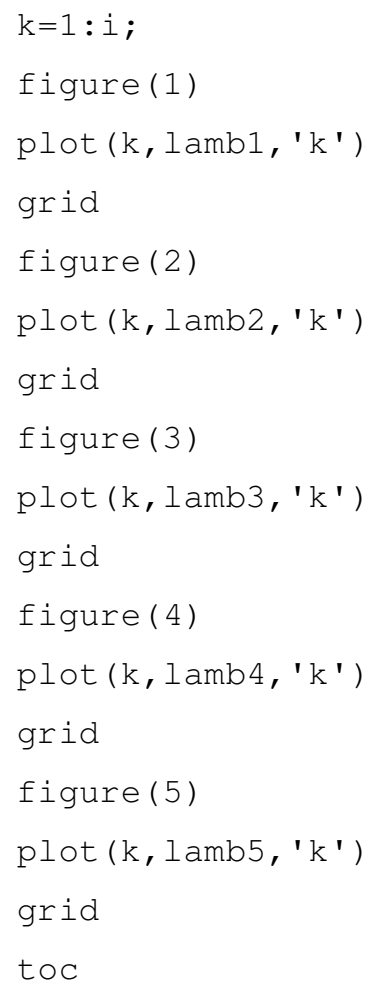


Functions usadas para o cálculo dos expoentes de Lyapunov

\% Sistema não Linear \%

function $[x$ dot $]=1$ yapnovol $(t, x)$

global xo E ro L $r$ b h me M P2 d2 e G1 G2 G3 G4 G5 G6 G7 sd F omega Ie $\mathrm{T} A \ldots$

beta alfa R1 R2 sigma H S U V W K J Q Z psil D aa

Constantes :

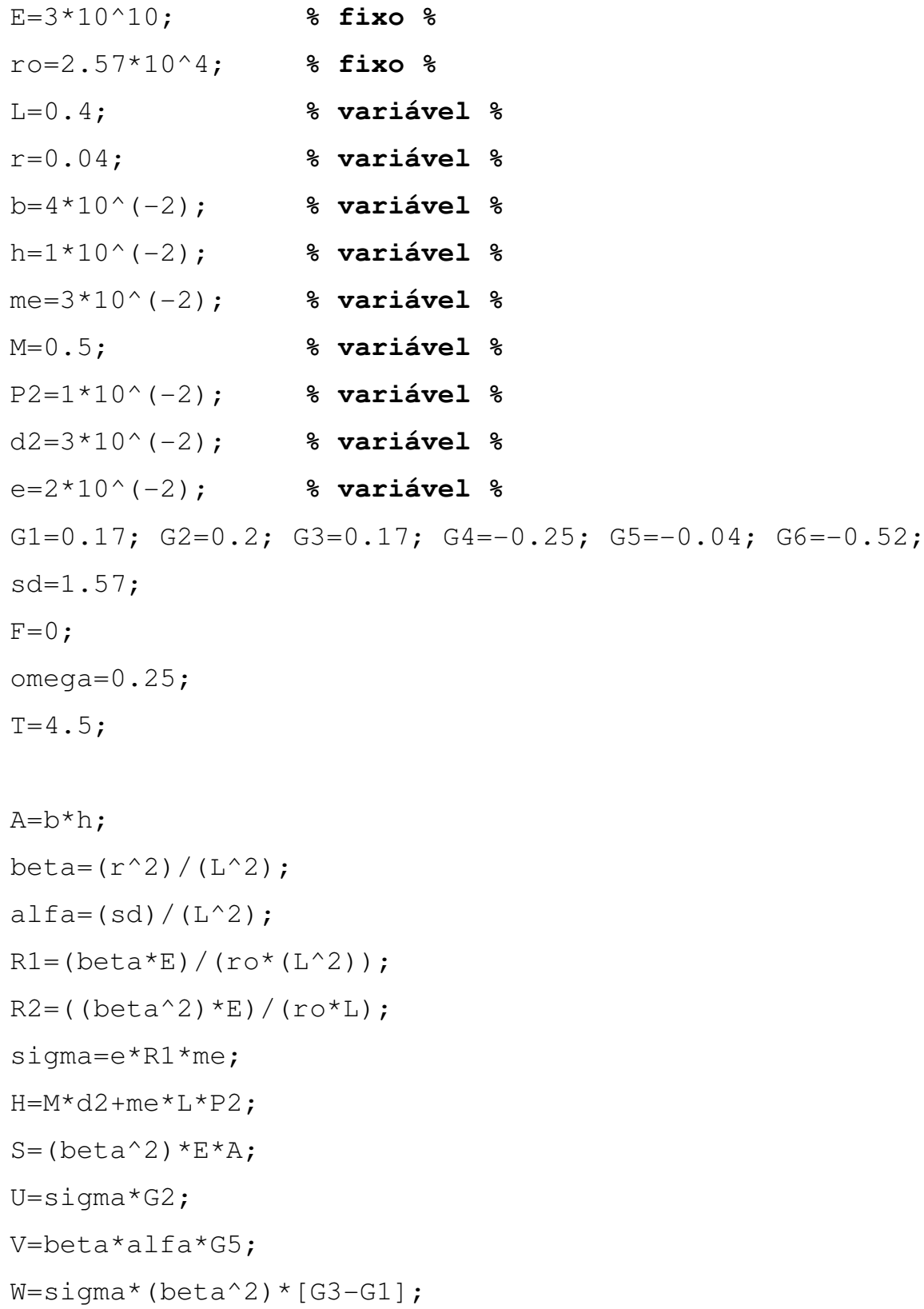




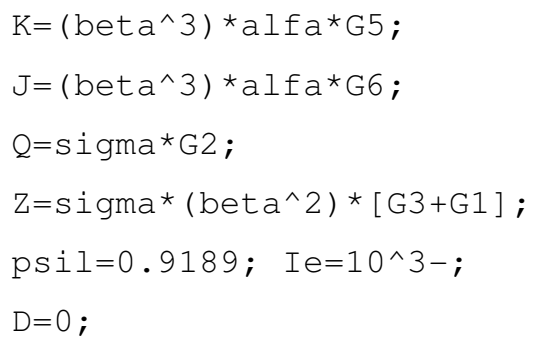

\section{\% Sistema linearizado \%}

function $[$ xdot $]=$ lyapnovo2 $(t, x)$

global xo E ro L $r$ b h me M P2 d2 e G1 G2 G3 G4 G5 G6 G7 sd F omega Ie T A ...

beta alfa R1 R2 sigma H S U V W K J Q Z psil D aa

\section{을 Contes \%}

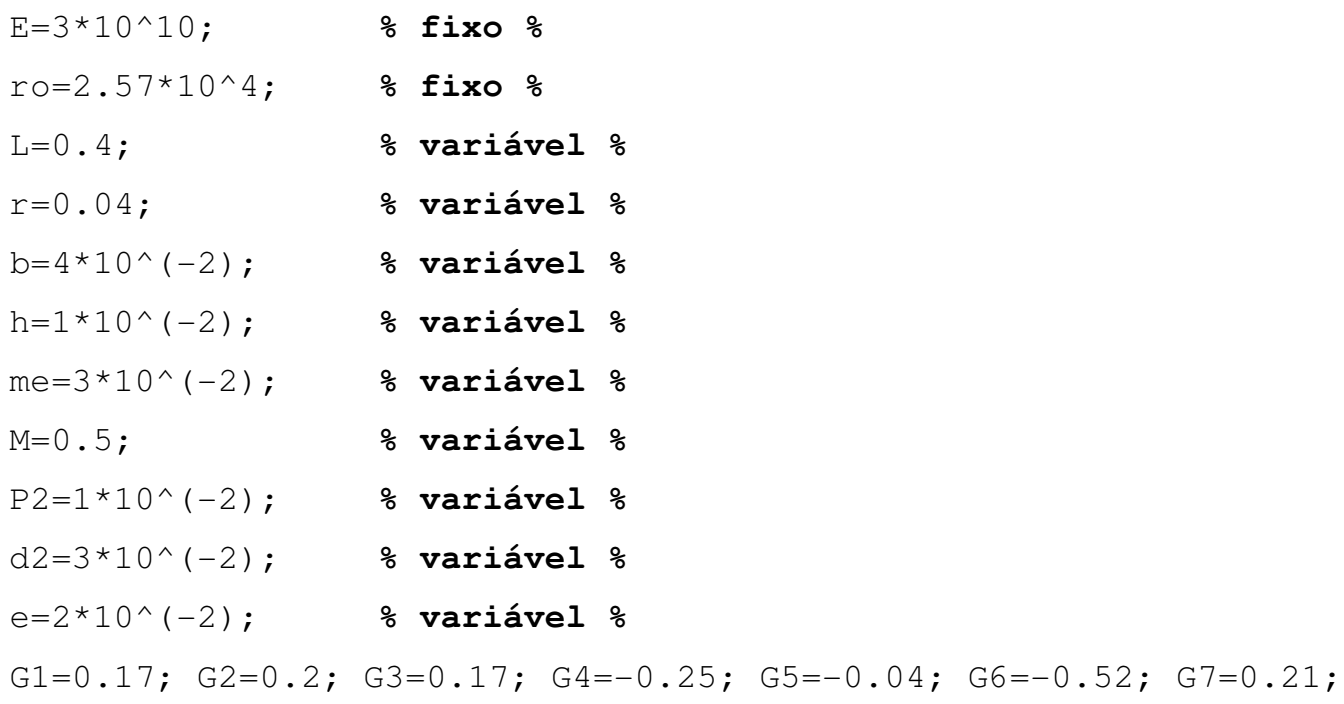




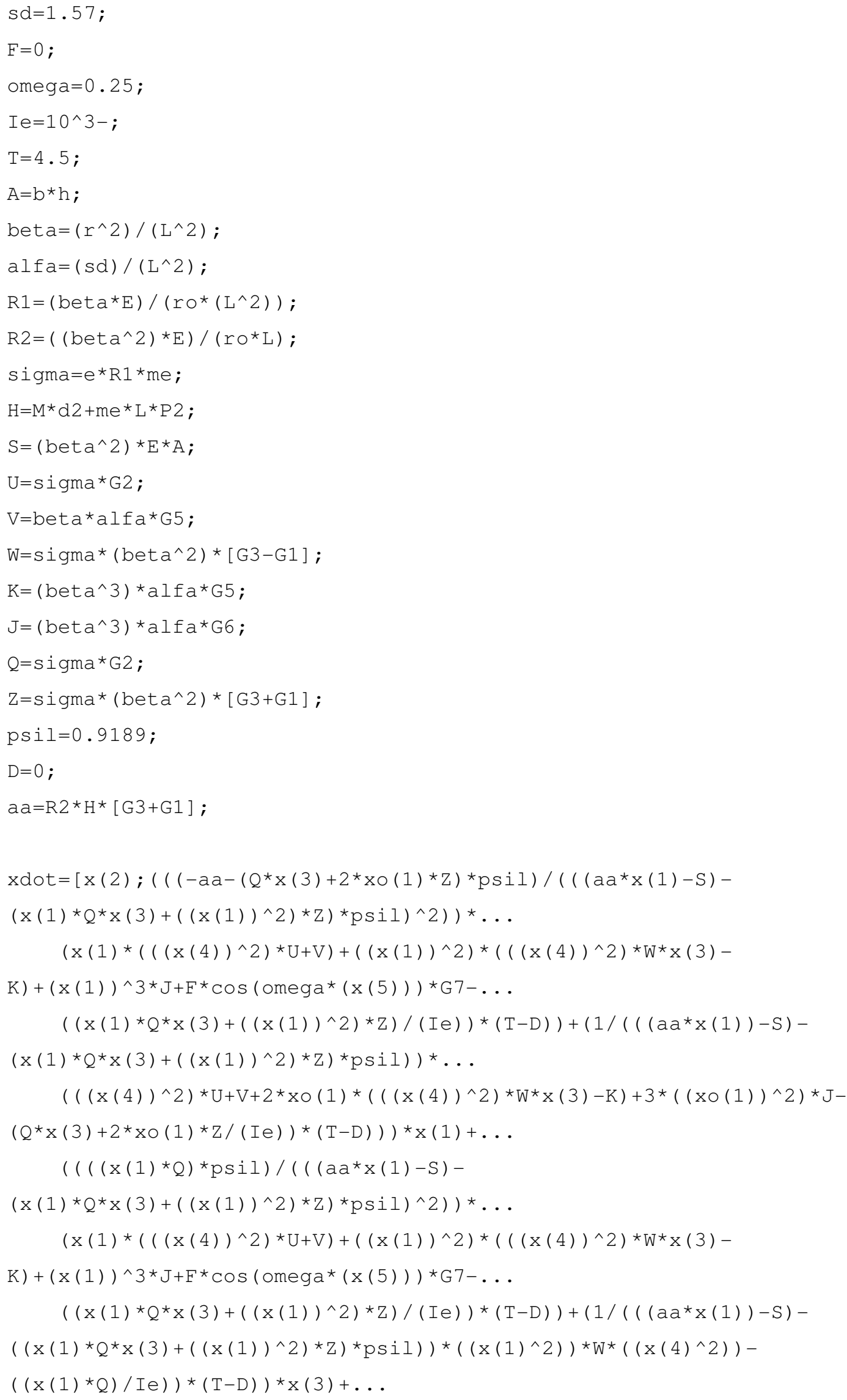


$\left(\left(1 /\left(\left(\left(a a^{*} x(1)\right)-S\right)-\right.\right.\right.$

$(x(1) * Q * x(3)+((x(1)) \wedge 2) \star z) * \operatorname{psil})) \star((x(1) \star 2 * x o(4) \star U)+((x(1)) \wedge 2) \star 2 * x o(4)$

$\left.\left.{ }^{\star} W{ }^{*} \mathrm{X}(3)\right)\right){ }^{*} \mathrm{X}(4)+\ldots$

$\left(\left(1 /\left(\left(\left(a a^{*} x(1)\right)-S\right)-(x(1) * Q * x(3)+((x(1)) \wedge 2) * z) * p s i 1\right)\right) *(-\right.$

$\mathrm{F}^{\star} \operatorname{Sin}\left(\right.$ omega*xo(5)) ${ }^{\star}$ omega $\left.\left.{ }^{\star} \mathrm{G} 7\right)\right){ }^{*} \mathrm{x}(5) ; \mathrm{x}(4) ; \ldots$

$(((-\mathrm{a} a+(\mathrm{Q} * \mathrm{x}(3)+2 * \mathrm{xo}(1) * \mathrm{Z}) * \mathrm{psil}) /(((\mathrm{a} a * \mathrm{x}(1)-\mathrm{S})-$

$\left.\left.\left.\left(\mathrm{x}(1){ }^{*} \mathrm{Q} * \mathrm{x}(3)+\left((\mathrm{x}(1))^{\wedge} 2\right) \star \mathrm{Z}\right) \star \mathrm{psil}\right) \wedge 2\right)\right) \star((\mathrm{x}(1) \star(((\mathrm{x}(4)) \wedge 2) \star \mathrm{U}+\mathrm{V})+\ldots$

$\left.\left((x(1))^{\wedge} 2\right) *\left(\left((x(4))^{\wedge} 2\right) * W^{\star} x(3)-K\right)+(x(1))^{\wedge} 3 * J\right) *(-p s i l)$

$+\mathrm{F}^{\star} \operatorname{Cos}($ omega* $\left.(x(5))) * \mathrm{G} 7-((((\mathrm{x}(1) * \mathrm{a} a)-\mathrm{S}) *(\mathrm{~T}-\mathrm{D}) /(\mathrm{Ie})))\right)+\ldots$

$\left(1 /\left(\left(\left(a a^{\star} x(1)\right)-S\right)-\right.\right.$

$(\mathrm{x}(1) \star \mathrm{Q} * \mathrm{x}(3)+((\mathrm{x}(1)) \wedge 2) \star \mathrm{z}) * \mathrm{psil})) \star\left(\left(\left((\mathrm{x}(4))^{\wedge} 2\right) \star \mathrm{U}\right)+\mathrm{V}+2 \star \mathrm{xo}(1) \star\left(\left((\mathrm{x}(4))^{\wedge} 2\right.\right.\right.$

)$\left.\left.\left.* W^{*} \mathrm{x}(3)-\mathrm{K}\right)+3 *((\mathrm{x} O(1)) \wedge 2) * \mathrm{~J}\right)\right) *(-\mathrm{psil})-\ldots$

$((\mathrm{a} a *(\mathrm{~T}-\mathrm{D})) / \mathrm{Ie})){ }^{*} \mathrm{x}(1)+(((\mathrm{x}(1) * \mathrm{Q}) * \mathrm{psil}) /(((\mathrm{a} a * \mathrm{x}(1)-\mathrm{S})-$

$\left.\left.\left(x(1) * Q^{*} x(3)+((x(1)) \wedge 2) * z\right) * \operatorname{psil}\right)^{\wedge} 2\right) * \ldots$

$\left(\left(\left(x(1) *\left((x(4))^{\wedge} 2\right) * U+V\right)+\left(\left(x(1)^{\wedge} 2\right) *\left(\left((x(4))^{\wedge} 2\right) * W^{\star} x(3)-\right.\right.\right.\right.$

$\left.\mathrm{K}))+\left(\mathrm{x}(1)^{\wedge} 3\right) * \mathrm{~J}\right) *(-\mathrm{psil})+\mathrm{F}^{*} \cos (\operatorname{omega} * \mathrm{x}(5)) * \mathrm{G} 7-\ldots$

$(((x(1) * a a)-S) *(T-D)) /(I e))+(1 /((a a * x(1))-S)-$

$\left.\left.\left(x(1) * Q * x(3)+\left((x(1))^{\wedge} 2\right) \star Z\right) * \operatorname{psil}\right) *\left(x(1)^{\wedge} 2\right) \star(x(4))^{\wedge} 2 * W\right) \star x(3)+\ldots$

$((1 /((a) * x(1))-S)-$

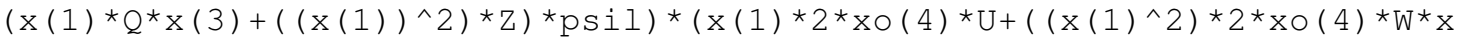

(3))) $) \star_{x}(4)+\ldots$

$\left(\left(1 /\left(\left(a a^{*} x(1)\right)-S\right)-(x(1) * Q * x(3)+((x(1)) \wedge 2) * z) * p s i l\right) *(-\right.$

$\mathrm{F}^{*} \sin ($ omega*xo(5))*G7*omega) )*x(5);0]; 
C.2 - Programa Utilizado para o Cálculo do Histórico no Tempo e Plano de Fase.

\% Programa para calcular histórico no tempo, plano de fase de uma viga excitada por um motor não ideal \%

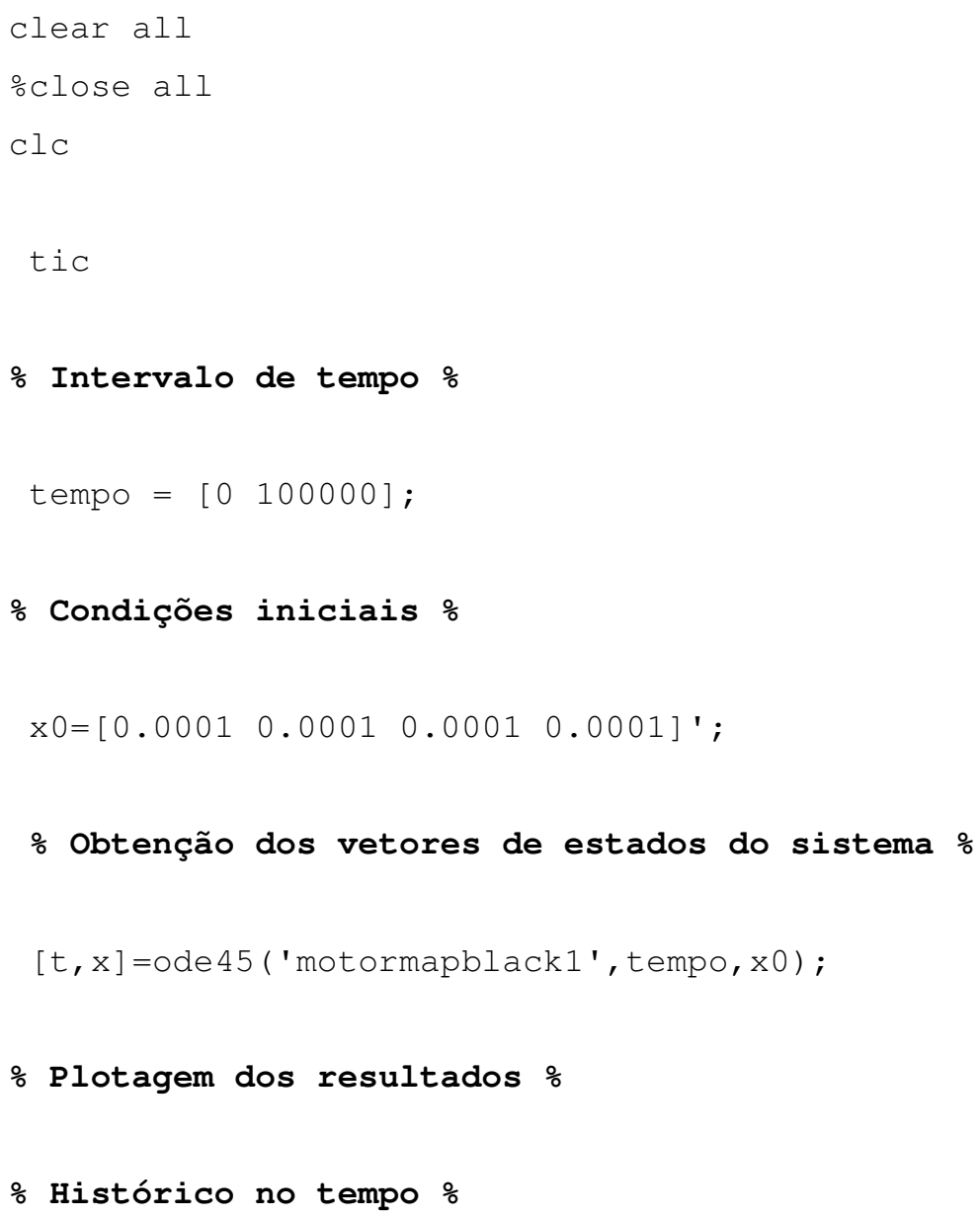




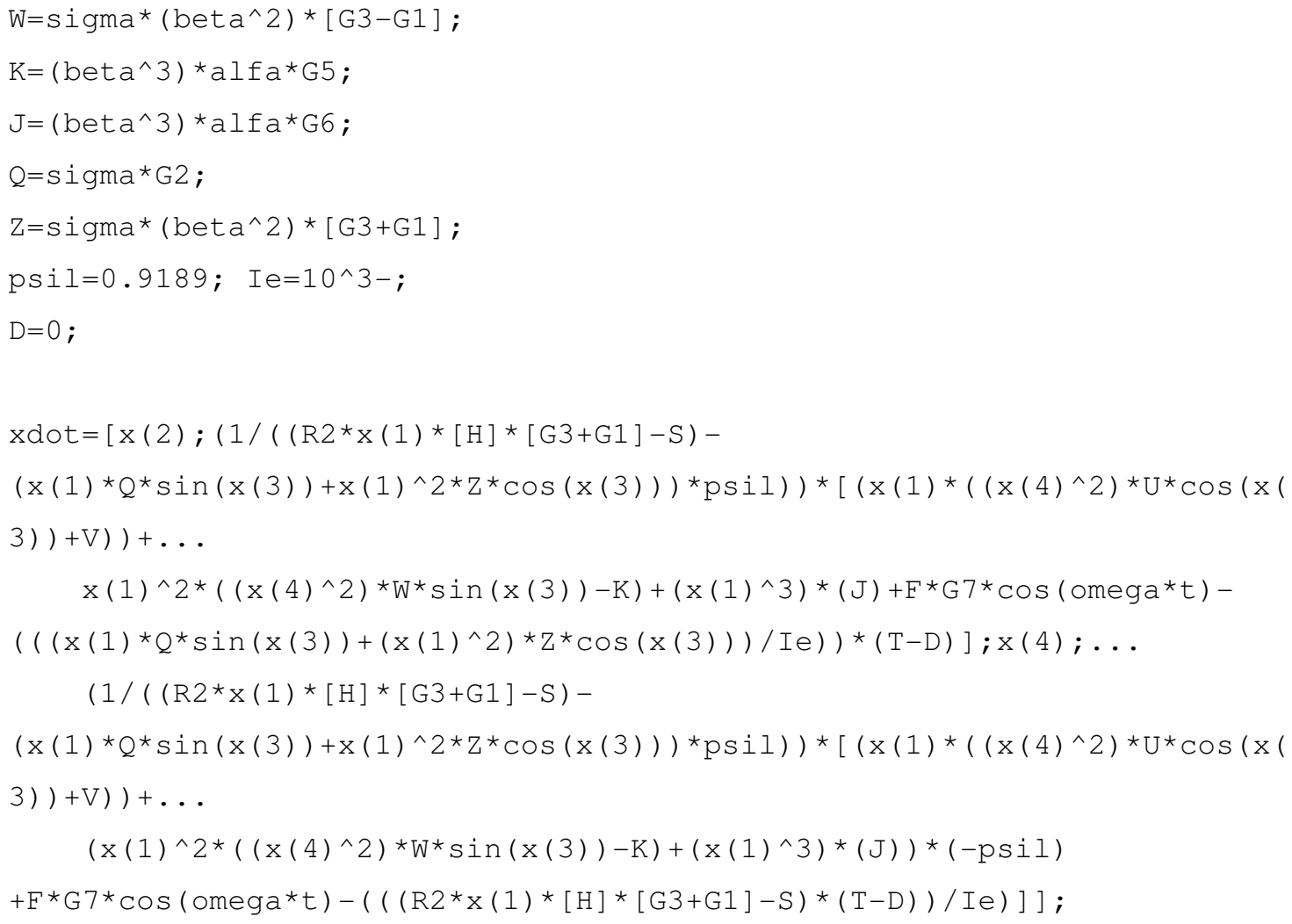

\section{C.3 - Programa Utilizado para a Obtenção do Gráfico do Salto.}

\% Programa para calcular o Salto - Efeito Sommerfeld \%

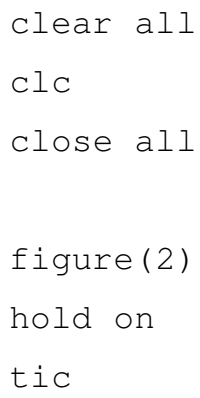


\% Condições iniciais \%

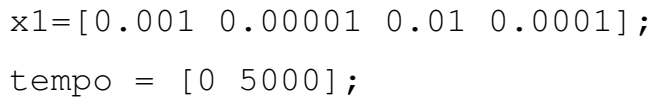

\% Obtenção do vetor de estados do sistema \%

$[t, x]=$ ode23 ('diagmapmotor 1 ', tempo, x1) ;

\section{\% Eliminação da parte transiente \%}

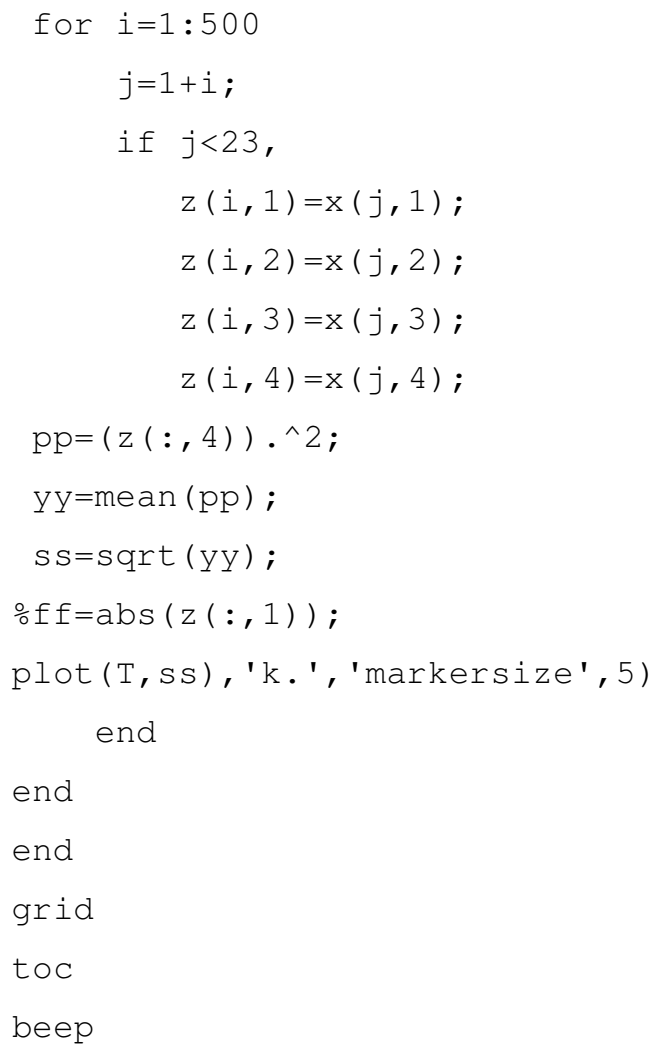




\section{Function usada para a obtenção do gráfico do salto.}

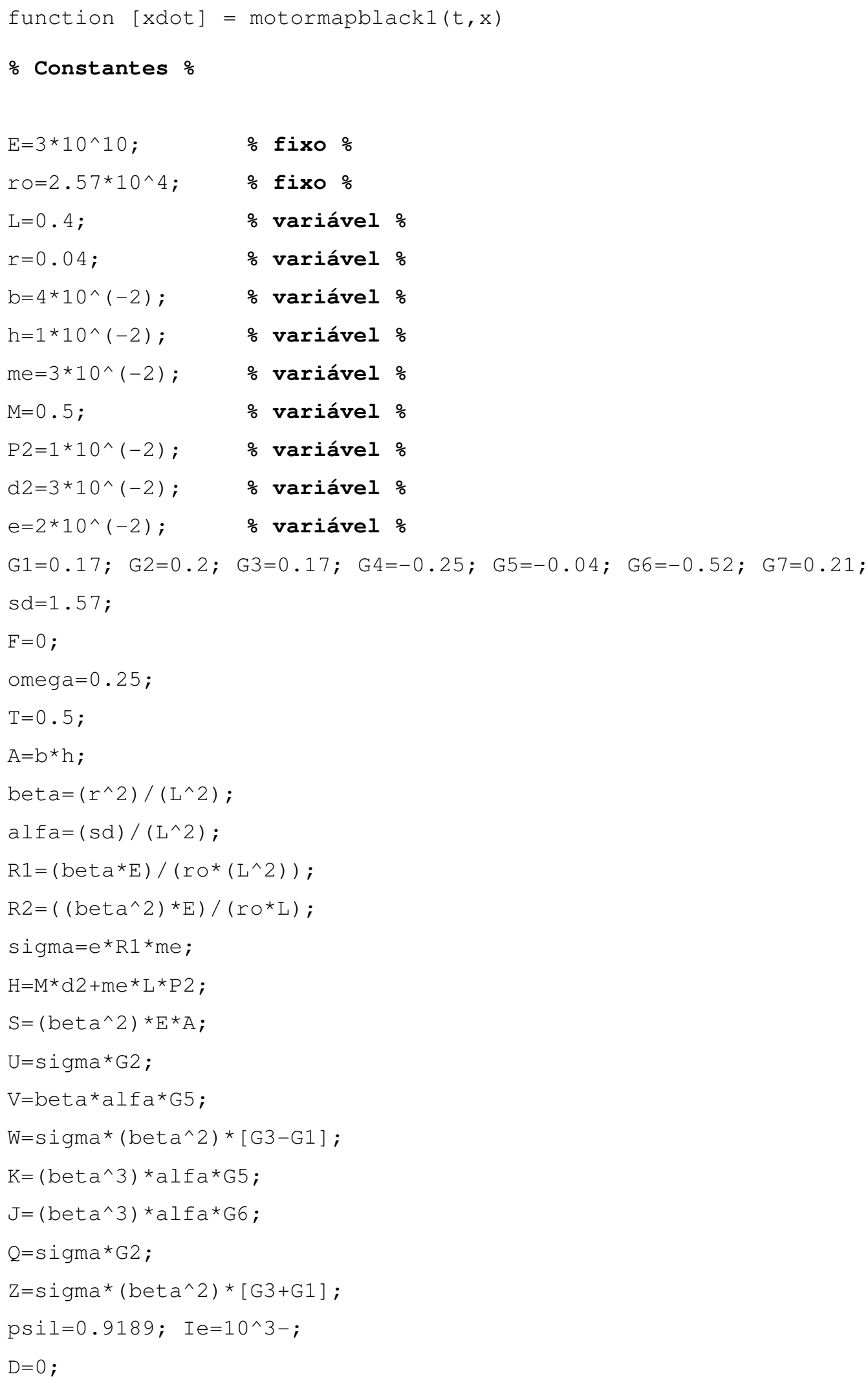


$x d o t=[x(2) ;(1 /((R 2 * x(1) *[H] *[G 3+G 1]-S)-$

$\left.\left.\left(x(1) \star Q * \sin (x(3))+x(1)^{\wedge} 2 * z * \cos (x(3))\right) \star \operatorname{psil}\right)\right) \star[(x(1) \star((x(4) \wedge 2) \star U * \cos (x($ 3) $)+\mathrm{V}))+\ldots$

$\mathrm{x}(1)^{\wedge} 2 \star\left(\left(\mathrm{x}(4)^{\wedge} 2\right) \star \mathrm{W}^{\star} \sin (\mathrm{x}(3))-\mathrm{K}\right)+\left(\mathrm{x}(1)^{\wedge} 3\right) \star(J)+\mathrm{F}^{\star} \mathrm{G} 7{ }^{\star} \cos ($ omega $* \mathrm{t})-$

$\left.\left(\left(\left(x(1) * Q * \sin (x(3))+\left(x(1)^{\wedge} 2\right) * Z * \cos (x(3))\right) / I e\right)\right) *(T+x(4)-D)\right] ; x(4) ; \ldots$

$(1 /((\mathrm{R} 2 * x(1) *[\mathrm{H}] *[\mathrm{G} 3+\mathrm{G} 1]-\mathrm{S})-$

$\left.\left.\left(x(1) * Q^{\star} \sin (x(3))+x(1)^{\wedge} 2 * Z^{*} \cos (x(3))\right) * \operatorname{psil}\right)\right) \star\left[\left(x(1) \star\left((x(4) \wedge 2) * U^{\star} \cos (x(\right.\right.\right.$ 3) $)+\mathrm{V}))+\ldots$

$\left(x(1)^{\wedge} 2 *\left(\left(x(4)^{\wedge} 2\right) * W^{\star} \sin (x(3))-K\right)+\left(x(1)^{\wedge} 3\right) *(J)\right) *(-p s i l)$

$+F * G 7 * \cos ($ omega*t $)-(((R 2 * x(1) *[H] *[G 3+G 1]-S) *(T+x(4)-D)) / I e)]]$; 